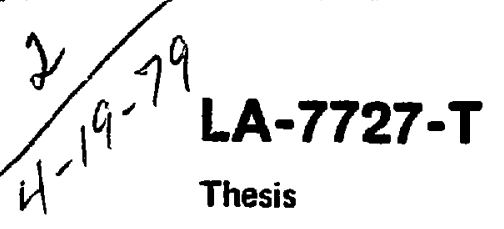

1

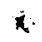
Photoionization Mass Spectrometric Studies
of Selected Compounds in a Molecular Beam 
LA-7727-T

Thesis

Special Distribution

Issued: March 1979

\section{Photoionization Mass Spectrometric Studies of Selected Compounds in a Molecular Beam}

Wayne Merle Trott*

"Sandia Laboratories, Org. 4212, Albuquerque, NM 87115.

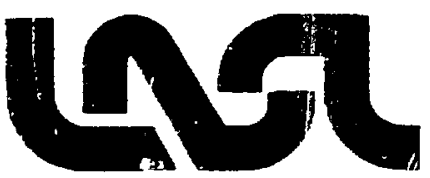

This teport was prepared as an account of work sponsusted by the United Siates Govemment. Netthet the

United States nor the United States Department of

Energy, no' any' of their employees, not any of their

contractors, subcontractors, of their emiployees, makes

any warranty, express or implied, or ssumes any legat

liabilly or sesponsibility for the accury completeness

or usefulnes of any th formation, apparatus, proulucl or

process disclosed, or represents that its use would not

infringe privately owned rights. 
TABLE OF CONTENTS

Page

List of Figures... . . . . . . . . . .

viii

List of Tables . . . . . . . . . . . . . X xi

I. Introduction: General Methods in the Study of Ionization and Dissociation Processes . . . . I

II. Photoionization Mass Spectrometry . . . . . 12

A. Historical overview . . . . . . . . 12

B. General Considerations ........ . 17

1. Phctoionization Processes..... . 18

a. Direct Ionization and the Franck-Condon Princlple . . . 18

b. Selection Rules......... . 22

c. Autoionization. . . . . . . 29

2. Photoionization Efficlency Curves . . 36

a. Physical Conditions leading to Coarse and Fine Structure. . . . . . 37

b. Molecular and Ionic State Energetic Information .........

c. Instrumental and Thermal Effects. $\equiv 5$

3. Appearance Potential Measurements of Fragmentation Processes . . . . . . 52 
C. Supersonic Molecular Beam Sampling. . . . i $_{4}$

D. Experimental Design . . . . . . . . . 95

III. Apparatus . . . . . . . . . . . . . . . . I III

A. Nozzle Beam Source and Ionizur. . . . . . III

B. Monochromatic Vacuum Ultraviolet Source . . 116

C. Mass Analysis and Ion Detection . . . . 129

D. Apparatus Performance Calculations. . . . 134

E. Data Collection Procedures. . . . . . . 242

IV. Results and Discussion. . . . . . . . . . 147

A. Preliminary Observations with Electron

Impact. . . . . . . . . . . . . 147

B. Photoionization of Acetone and

Acetone-d $\alpha_{6} \cdot$. . . . . . . . . . . 147

1. Experimental. . . . . . . . . 150

2. Results . . . . . . . . . . 152

3. Discussion. . . . . . . . . . . 153

a. Acetone and Acetone-d6

Monomer Ions. . . . . . . . . 253

b. $\mathrm{CH}_{3} \mathrm{CO}^{+}$and $\mathrm{CD}_{3} \mathrm{CO}^{+}$Fragment Ions . . 163

c. Acetone and Acetone- $d_{6}$

Cluster Ions. . . . . . . . I60

c. Preliminary Photoionization Experiments on Carbon D1sulf1de . . . . . . . . . . . 179 
I. Experimental . . . . . . . . 180

2. Results and Discussion....... . 182

v. Recommendations. ............ 190

References................. 200 


\section{LIST OF FIGURES}

F1gure

Page

1 Photolonization Cross Section for Helium (from Reference 118)........... .

2 Calculated Franck-Condon Factors and Idealized Photoionization Efficiency Curves Corresponding to Varlous Changes in Bond Length for a Single Oscillator (from Reference 1). . . . . . .

3 Photoionization Efficiency Curve for Argon (from Reficrence 73)............

4 Photolonization Efficiency Curve for NO (from Reference 89). . . . . . . . . .

5 Photolonization Efficiency Curve for $\mathrm{CS}_{2}{ }^{122}$ (from Reference 3) . . . . . . . . . 52

6 Variation of Vibrational, Rotational and Translational (Paraliel and Perpendicular to Expansion Ax1s) Temperatures in a Free Jet as a Function of Axlal Distance, $x$, In Nozzle Diameters $D_{N}$ (from Reference 167 )

7 Effect of Skimmer and End Wall Teinperatures on Argon Monomer and Dimer Beam Intensity (from Reference 199) . . . . . . . . . 


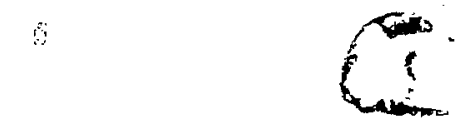

$8 \mathrm{H}_{2}$ Spectrum Between 850 and $1750 \AA$. Lamp Pressure, 250н; Discharge Current, $400 \mathrm{~mA}$. Pt-Coated Grating, 1200 lines/mm (from Reference 209). . . . . . . . 98

9 Nozzle Beam Sampling and Ion Source . . . . 112

10 Schematic Diagram of Photoionization Mass Spectrometer. . . . . . . . . . 117

$11 \mathrm{H}_{2}$ Spectrum Between 1160 and $1280 \AA$. Lamp Pressure, 1000u; Discharge Current, $300 \mathrm{~mA}$. $\mathrm{MgF}_{2}$-Coated Grating, 2400 lines/mm; $200 \mathrm{u}$ Entrance and Exit Slits ........

12 Schematic Representation of Major Operating and Data Collection Electronics for the Photolonization Mass Spectrometer . . . . 144

13 Photolon Yield Curve for $\left(\mathrm{CH}_{3} \mathrm{COCH}_{3}\right)^{+}$ from 1240 to $1285 \AA$. . . . . . . . . . IE-

14 Photolon Yield Curve for $\left(\mathrm{CD}_{3} \mathrm{COCD}_{3}\right)^{+}$

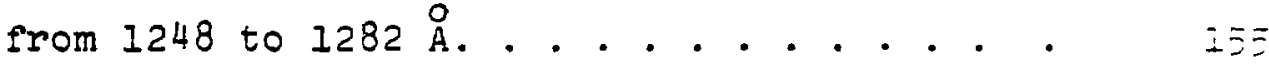

15 Appearance Potential Curves for $\mathrm{CH}_{3} \mathrm{CO}^{+}$and $\mathrm{CD}_{3} \mathrm{CO}^{+}$from 1148 to $1283 \AA . . . . . . . \quad \Sigma \equiv 0$

16 Photolon Yield Curve for $\left(\mathrm{CH}_{3} \mathrm{COCH}_{3}\right)_{2}^{+}$ from 1239 to $1351 \AA . . . . . . . . . . \quad 157$

17 Photolon Yleld Curve for $\left(\mathrm{CD}_{3} \mathrm{COCD}_{3}\right)_{2}^{+}$ from 1270 to $1349 \AA \ldots \ldots$. . . . . . 153 
18 Photoion Yield Curve for $\left(\mathrm{CH}_{3} \mathrm{COCH}_{3}\right)_{3}^{+}$

from 1334 to 1369 凡.............. 159

19 Photoion Yield Curve for $\left(\mathrm{CH}_{3} \mathrm{COCH}_{3}\right)_{4}^{+}$

from 1324 to 1379 \&.............. 160

20 Appearance Potential Curve for $\left(\mathrm{CH}_{3} \mathrm{COCH}_{3}\right) \cdot \mathrm{CH}_{3} \mathrm{CO}^{+}$ from 1190 to 1241 \&.............. 161

21 Plot of the Observed Ionization Energies for $\left(\mathrm{CH}_{3} \mathrm{COCH}_{3}\right)_{n}, \mathrm{n}=1-4$, as a Function of $1 / n \cdot \ldots, 176$

22 Photolon Yield Curve for $\mathrm{CS}_{2}{ }^{+}$from 1203 to $1239 \AA$. . . . . . . . . . . . . 183

23 Photoion Yield Curve for $\left(\mathrm{CS}_{2}\right)_{2}{ }^{+}$ from 1007 to 1292 \&................. 185 


\section{IIST OF TABLES}

Table

Page

I Gain of EMI 9642/2B Electron Multiplier

as a Function of Cathode Potential. . . . . 133

II Summary of Electron Bombardment Results

for Various Compounds in Molecular Beams . . 1-E

II Summary of Energetic Information for

Acetone and Acetone-d 6 Parent, Fragment

and Cluster Ions... . . . . . . . . . I62

IV Calculated Acetone Dimer Binding Energies

from $B_{2}(T)$ Data . . . . . . . . . . . 173

$V$ Calculated Carbon Disulfide Dimer Binding

Energy from $B_{2}(\mathrm{~T})$ Data. 293 . . . . . . . 186

VI Ions For Which Photoionfation Efficiency

Curves Have Been Determined Using Supersonic

Molecular Beam Mass Spectrometry . . . . . . . - 


\title{
PHOTOIONIZATION MASS SPECTROMETRIC STUDIES OF SELECTED COMPOUNDS IN A MOLECULAR BEAM
}

\author{
Wayne Merle Trott, Ph.D. \\ Department of Chemistry \\ The University of New Mexico, 1978
}

Photolonization efficiency curves have been measured at moderate to high resolution for several species produced In supersonic molecular beams of acetone, acetone- $d_{6}$ and $\mathrm{CS}_{2}$. The molecular beam photoionization mass spectrometer which has been assembled for this work is described. The performance of this instrument has been characterized by a number of experiments and calculations.

As a result of adiabatic cooling during the nozzle expansion, photoion yield curves have been obtained for $\mathrm{CH}_{3} \mathrm{COCH}_{3}{ }^{+}$and $\mathrm{CD}_{3} \mathrm{COCD}_{3}{ }^{+}$at an effective resolution of approximately $12 \mathrm{meV}$. Partial condensation was objerved in the acetone beam to the extent that sufficient concentrations of $\left(\mathrm{CH}_{3} \mathrm{COCH}_{3}\right)_{2},\left(\mathrm{CD}_{3} \mathrm{COCD}_{3}\right)_{2},\left(\mathrm{CH}_{3} \mathrm{COCH}_{3}\right)_{3}$ and $\left(\mathrm{CH}_{3} \mathrm{COCH}_{3}\right)_{4}$ were formed to permit the study of their photolonization efficiency behavior as well. Appearance potentlaI curves have been determined for $\mathrm{CH}_{3} \mathrm{CO}^{+}, \mathrm{CD}_{3} \mathrm{CO}^{+}$ and $\left(\mathrm{CH}_{3} \mathrm{COCH}_{3}\right) \cdot \mathrm{CH}_{3} \mathrm{CO}^{+}$sragments. The measured ionization potentials of acetone and acetone-ds monomers are 
$9.694 \pm 0.006 \mathrm{eV}$ and $9.695 \pm 0.006 \mathrm{eV}$ respectively. Transitions to higher vibrational levels in $\mathrm{CH}_{3} \mathrm{COCH}_{3}{ }^{+}$are seen at 320,695 and $930-1370 \mathrm{~cm}^{-1}$ above threshold. The effect of perdeutero substitution is to reduce these frequencles to 260 and $660-1100 \mathrm{~cm}^{-1}$. Appearance potentlals of $\mathrm{CH}_{3} \mathrm{CO}^{+}$and $\mathrm{CD}_{3} \mathrm{CO}^{+}$fragments are observed at $10.52 \pm 0.02 \mathrm{eV}$ and $10.56 \pm 0.02 \mathrm{eV}$ respectively. This study reports the first observation of a simple functional depenaence of ionization potential with cluster size. The measured lonization energies for $\left(\mathrm{CH}_{3} \mathrm{COCH}_{3}\right)_{n}, \mathrm{n}=1-4$, are found to decrease linearly as a function of $1 / n$. This $1 / n$ functional dependence compares favorably to that which would be expected on the basis of a straightforward perturbation model for the cluster ion system. Ionization thresholds for $\left(\mathrm{CH}_{3} \mathrm{COCH}_{3}\right)_{2},\left(\mathrm{CH}_{3} \mathrm{COCH}_{3}\right)_{3}$ and $\left(\mathrm{CH}_{3} \mathrm{COCH}_{3}\right)_{4}$ are seen at $9.26 \pm 0.03 \mathrm{eV}, 9.10 \pm 0.03 \mathrm{eV}$ and $9.02 \pm 0.03 \mathrm{eV}$ respectively. Within experimental resolution, the ionization potentials of $\left(\mathrm{CH}_{3} \mathrm{COCH}_{3}\right)_{2}$ and $\left(\mathrm{CD}_{3} \mathrm{COCD}_{3}\right)_{2}$ are identical. The appearance potential of the process $\left(\mathrm{CH}_{3} \mathrm{COCH}_{3}\right)_{2}+\left(\mathrm{CH}_{3} \mathrm{COCH}_{3}\right) \cdot \mathrm{CH}_{3} \mathrm{CO}^{+}+\mathrm{CH}_{3}+e^{-}$ is found to be $10.08 \pm 0.05 \mathrm{eV}$. By consideration of appropriate thermodynamic cycles, a lower bound for the acetone dimar lon binding energy is calculated to be $0.538 \mathrm{eV}(12.4 \mathrm{kcal} / \mathrm{mole})$ and the desolvation energy of 
$\left(\mathrm{CH}_{3} \mathrm{COCH}_{3}\right) \cdot \mathrm{CH}_{3} \mathrm{CO}^{+}$is estimated to be $0.544 \mathrm{eV}(12.5$ $\mathrm{kcal} / \mathrm{mole}$ ).

The photolon yleld curve of $\mathrm{CS}_{2}{ }^{+}$has been measured between 1200-1240 $\AA$ at an optical resolution of about $30 \mathrm{meV}$. The $\left(\mathrm{CS}_{2}\right)_{2}{ }^{+}$curve has also been determined in the wavelength range 1000-1290 \&. The observed ionization potentials for the monomer and dimer species are $10.056 \pm 0.015 \mathrm{eV}$ and approximately $9.63 \mathrm{eV}$ respectively. These values in conjunction with an estimate of the dimer binding energy from second virlal coefficlent data are used to calculate a lower bound for the $\left(\mathrm{CS}_{2}\right)_{2}{ }^{+}$binding energy of $11.0 \mathrm{kcal} / \mathrm{mole}$.

Photolonization

Molecular Beams

Clusters

Ace tone

Carbon Disulfide 
I. INTRODUCTION: General Methods in the Study of Ionization and Dissoclation Processes

The interaction of gaseous neutral molecules and various lonizing agents is a fundamental subject of chemical physics. Of particular interest are those quantities pertaining to the energetics and kinetics of positive ion formation. In order to obtain accurate Information concerning these properties, a substantial number of experimental methods and data interpretation technlques have been developed. These data measurement and Interpretation processes have been discussed and contrasted in considerable detail.1-7 Examination of recent positive ion Ifterature ${ }^{8}$ would seem to indicate that four general technlques have been most useful: ultraviolet absorption spectroscopy, electrón impact methods, electron spectroscopy and photoionization. For many molecules, ultraviolet absorption spectroscopy provides the most accurate fonization potential data. This experimental technique involves the measurement of total absorption cross section as a function of photon energy. At appropriate energies, excitation of ground state electrons into molecular orbitals of highen glartum number may occun, productna 
corresponding absorption lines. Where it is possible to 1dentify a Rydberg absorption band system, the band position $v_{n}$ of the members of the series can generally be fit to the modified Rydberg equation:

$$
v_{n}=v_{\infty}-\frac{R}{(n-\delta)^{2}}
$$

where $v_{\infty}$ is the ionization limit to which tha series converges, $\mathrm{R}$ is the Rydberg constant $\left(109,737.312 \mathrm{~cm}^{-1}\right)$, $\mathrm{n}$ is the princlpal quantum number of the Rydberg orbital of wave number $v_{n}$, and $\delta$ is the so-called quantum defect. Depending on the complexity of the absorption spectra, one or more Rydberg serles may be found which converge to various vibrational levels in the ground and higher electronic states of the ion. Due to the high resolution at which atomic and molecular absorption lines can be measured (often better than $\pm 0.1 \AA$ ), it is frequently possible to obtain very accurate values for the first adiabatic ionization potential. A considerable body of experimental data has resulted from utilization of this technique. ${ }^{9}$

Although extremely accurate information pertaining to various lonization limits is often accessible through ultraviolet absorption spectroscooy, this experimental method is somewhat limited in scope. Especially for polyatomic molecules, interpretation of the spectra is frequently rendered dffflcult on impossible as a result of absorption. 
Ine broadenting due to predissoclation or as a result of pseudo-continuous spectra arising from various competing Rydberg transitions. The saturated hydrocarbons appear to be particularly intractable in this regard, inasmuch as no Rydberg absorption band systems have been found for this class of molecules. 10 The vacuum ultraviolet spectrum of acetone provides another interesting case of difficult interpretation. On the basis of intensity, three absorption bands were assigned by Duncan to a Rydberg series converging to an ionization potential of $10.26 \mathrm{eV} .^{11} \mathrm{~A}$ subsequent photoionization experiment by Watanabe ${ }^{12}$ indicated a much lower ionization potential of $9.69 \mathrm{eV}$. Using the photolonization value as a reference, Watanabe was able to reanalyze the spectrum and identify another series converging to $9.705 \mathrm{eV}$. Finally, in addition to difflculties in spectrum interpretation, it is important that comparatively little information concerning dissoclation processes is obtainable using ultraviolet absorption spectroscopy. Thus, it is obvious that complementary experimental techniques are needed to provide a more complete understanding of positive ion energetics and kinetics.

The various electron impact techniques are widely used methods for studying lonization and dissociation mechanisms. When a beam of electrons of sufficient energy is passed through a sas, a number of competitive ard consecutive processes may occur. Major processes resulting in positive 
ion formation are as follows:
(I) $\mathrm{AB}+\mathrm{e}^{-} \rightarrow \mathrm{AB}^{+}+2 \mathrm{e}^{-}+\mathrm{KE}$
Direct Ionization
(2) $\mathrm{AB}+\mathrm{e}^{-} \rightarrow \mathrm{AB}^{++}+3 \mathrm{e}^{-}+\mathrm{KE}$
Multiple Ionization
(3) $A B+e^{-}+A B^{+}+2 e^{-}$
Parent Ion
$+A^{+}+B+2 e^{-}$
Fragmentation
(4) $A B+e^{-} \rightarrow A B^{*}+e^{-}$
Autolonization
$\rightarrow \mathrm{AB}^{+}+2 \mathrm{e}^{-}+\mathrm{KE}$
(5) $A B+e^{-} \rightarrow A B^{*}+e^{-}$
$+\mathrm{A}^{+}+\mathrm{B}^{-}+\mathrm{e}^{-}+\mathrm{KE}$ Ion Pair Production

Using electron Impact as an fonization source, lonization potentlals and appearance potentials are generally determined directly by plotting the ion current (usually mass selected) as a function of electron energy. From a number of theoretical approaches, ${ }^{13-18}$ it has been suggested that the electron bombardment ionization cross section should be, to a first approximation, a first order function of electron energy above threshold, with zero probability at onset. While there are exceptions, this prediction has been largely verifled experimentally 19 insofar as one generally observes, for simple ionization, a more or less asymptotic threshold followed by a roughly linear increase in lonization probability with excess energy. Careful interpretation of the lonfzation efficiency curve for each specie of interest may then be expected to yield a correct ionization or appearance potential. 
Principaliy due to the relative ease by which an intense beam of electrons may be produced over a wide energy range, electron impact techniques have enjoyed considerable popularity as a means of measuring ionization and dissociation energetics. There are however serfous problems which tend to limit the accuracy of these techniques. In particular, difficulties in obtaining a monoenergetic electron beam are well known.1,5,19 Problems involving interpretation of threshold behavior, 2,5 variable energy transfer during impact, 20,21 and pyrolysis effects ${ }^{22}$ are also significant. Considerable attention has been given to establishment of dependable ionization and appearance potential values by attempting to circumvent the various instrumental factors and by careful data interpretation. 23 Nevertheless, with more accurate low-energy experimental methods becoming increasingly avallable, it would appear that electron bombardment techniques are likely to be most viably applied to fonization and fragmentation studies above 25-30 eV (where the only competitive ionization source is synchrotron radiation), to experiments involving the generation of negative ions by resonance attachment, and to cases where electrons are capable of producing optically forbidden transitions. 3,24,25

Electron spectroscopy, a third general method for studyins ionization and dissociation pracesses, chlefly 
comprises three experimental techniques: photoelectron spectroscopy, Auger electron spectroscopy and Penning Ionization. These techniques involve the measurement of the kinetic energy distribution of electrons ejected from molecules which have been excited by UV photons, $\mathrm{X}$-ray photons, or metastable atoms respectively.

Photoelectron spectroscopy in particular has been widely employed in the study of molecular electronic structures. To briefly summarize the method, the kinetic energy analysis of the ejected photoelectrons takes advantage of the law of conservation of momentum in the process $A B+h v+A B^{+}+e^{-}+K E$ whereln the electron carries away the preponderance of the photon excess energy. To a very good approximation, the following relation then holds:

$$
E_{\text {electron }}=\text { hv }-E_{\text {ion }} i, v, f
$$

where Eelectron is the kinetic energy of the electron, hv is the photon excitation energy, and $E_{10 n}{ }^{i, v, f}$ is the energy needed to produce an ion in electronic state 1 with vibrational and rotational states $v$ and $f$ respectively. Hence, for a given photon beam energy, the accessible Ionlzation channels of the target molecule may be observed by analysis of the kinetic energy distribution of the ejected photoelectrons. An important alternative approach Is the resonant photolonization or threshold photoelectron spectroscopy technique in which the photon energy is varied whlle tuning the detector so as to accept only those 
electrons which have been efected with near zero kinetic energy. Electronic states whose cross sections decrease rapidiy as a function of excess energy are more readily observed in this way. The various experimental and analytical aspects of these methods have been reviewed in deta11.3,26-34

Electron and photon impact Ionization efficiency curves often exhibit autolonization structure which dominates that due to simple ionization processes. A principal advantage of conventional photoelectron spectroscopy is that autolonization is observed only when the incident light energy matches an autolonizing state. For light sources above $17 \mathrm{eV}$, this happens rarely because of the low density of exclted and lonic states in this energy region. 26 Similarly, threshold photoelectron experiments are af fected by autolonization processes only when the energy of the ejected electrons lies within the bandpass of the detector. Thus, with recent energy analyzer designs attaining resolutions of better than 10-15 meV, photoelectron spectroscopy and resonant photoionization methods are frequently capable of providing accurate and relatively unambiguous measurements of direct ionization thresholds. However, the inability to discriminate between those photoelectrons formed in dissociative processes and those arising from simple fontzation channels places a signiticant limitation on these techniques. 
The use of ultraviolet light to bring about ionization In a drect threshold experiment forms the basis for the fourth general approach to the study of positive ion energetics and kinetics. The major observable processes resulting from the absorption of a short wavelength photon are analagous to those arising from electron 1mpact:
(1) $\mathrm{AB}+\mathrm{hv}+\mathrm{AB}^{+}+e^{-}+\mathrm{KE}$ Direct Ionization
(2) $\mathrm{AB}+\mathrm{h \nu}+\mathrm{AB}^{++}+2 e^{-}+\mathrm{KE}$ Multiole Ionlzation : $\quad$ :

(3) $\mathrm{AB}+\mathrm{h \nu} \rightarrow \mathrm{AB}^{+}+\mathrm{e}^{-}$

Parent Ion

$$
\rightarrow A^{+}+B+e^{-}
$$

Fragmentation

(4) $A B+h \nu \rightarrow A B^{*}$

$$
\rightarrow \mathrm{AB}^{+}+\mathrm{e}^{-}+\mathrm{KE} \quad \text { Autolonization }
$$

(5) $A B+h \nu \rightarrow A B^{*}$

$$
\rightarrow \mathrm{A}^{+}+\dot{\mathrm{B}}^{-}+\mathrm{KE} \quad \text { Ion Pair Production }
$$

As with electron bombardment, the ionization efficiency curve (photoion current per photon current, plotted as a function of photon energy) generaliy provides straightforward lonization potential and appearance potentlal information. The use of ion mass analysis in conjunction with photoionization has virtually become standard practice insofar as it permits careful distinction between direct and dissociative ionization mechanisms and largely eliminates the possibility of error due to sample impurities. The recent widesoread acplication of photolonization mass spectrometry to the study of ionization and dissociation 
processes seems to be princlpally due to the fact that many of the disadvantages of electron bombardment techniques are eliminated through the use of ultraviolet radiation as an ionizing medium. In contrast to the largely first order threshold tehavior exhibited in electron impact ionization, it has been predicted

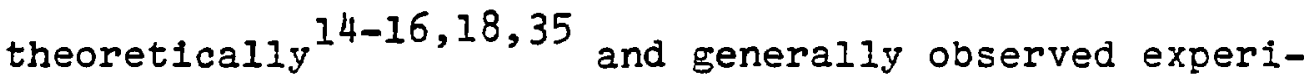
mentally ${ }^{36}$ that the cross section for a direct photoionization process approximates a zero order or step-like function of the excess lonizing energy. This oroperty facilitates accurate measurements of first and higher Ionization potentials and frequently allows the acquisition of Information pertaining to ionic vibrational freguencies, Franck-Condon factors for lonization, etc. The excellent energy resolution avaliable with current instrumental designs and the absence of effects due to vartable energy transfer and pyrolysis are additional factors which make photon impact an attractive source for lonization and fragmentation experiments. The development and use of phowionization methods has been the subject of severai reviews. 37-39

Experimental factors which have restricted the potential of photoionization mass spectrometric techniques in the past include low lon currents due to the lack of sufficlently intense vacuum ultraviolet light sources coupled with small shotoionization cross sections and 
wort-ing resolution as a result of the internal thermal energy of the sample molecules. The former difficulty has been largely overcome by instrumental developments resulting in increased useable photon fluxes and by improvements in ion detection sensitivities through the use of pulse counting techniques. Improvements in ult1mate resolution, on the other hand, have been achieved by cooling the sample gas directly or, very recently, by taking advantage of the adiabatic cooling properties of a supersonic nozzle expansion. The production of van der Waals molecules in sufficient concentrations to permit their investigation has proved to be an Important additional benefit to molecular beam sampling methods.

It is clear that photolonization mass spectrometry represents a poweriul experimental technique, especially when used in conjunction with a supersonic nozzle expansion. This thesis traces the historical development and reviews varlous experimental and analytical aspects of photolonization mass spectrometric methods. The characterIstics which promote molecular beams as a particularly sultable sampling source are discussed. The photoionization efficiency curves for several species produced in supersonic molecular beams of acetone, acetone-d6 and $\mathrm{CS}_{2}$ are reported. Fine structure in the data is analyzed in terms of molechlar and ionic electronic structures. 
Thermochemical quantities whlch have been derived from these data are also discussed. 


\section{PHOTOIONIZATION MASS SPECTROMETRY}

\section{A. Historical Overview}

The first experiments on photolonization of gases were performed shortly after the discovery of the surface photoelectric effect by Hertz in 1887. During the period 1900-1920, a number of studies were made on the effects of ultraviolet radiation on air and other compounds. 40 In general, it was found that ultraviolet light in the region 1250-1450 $\AA$ was effective in producing substantial ionization even though the ionization potentials of most of the constituents involved occur at wavelengths much lower than $1250 \AA$. Satisfactory explanations for these observations eluded the early investigators. Possible interpretations of these results include multistep Ionization channels, Ionization by accelerated photoelectrons or ionization of impurities such as mercury vapor. ${ }^{41}$

From 1923 to 1930 , the photolonization of aikali metal vapors was extensively studied. ${ }^{40}$ The relatively low lonization potentials of these compounds permitted the use of quartz optics and energy measurements were made with monochromatic 11ght sources. In 1929, mass analysis was introduced to detect photoiors from irradiated atomic potassium vapor. ${ }^{42}$ Terenin and Pooov veriormed the inst 
study of molecular ohotolonization processes using mass selection in 1932. 43 In this investigation, a simple magnetic mass analyzer unambiguously established the ion pair production processes in the photolonization of thallium halides.

The use of photolonization as a general technique for the study of positive ion energetics was frustrated by the lack of a method to produce well-characterized monochromatic photon energies in the range necessary for Ionization of most compounds. In 1950, however, the feasibility of energy measurements of dispersed radiation In the vacuum ultraviolet region was shown. 44 During the period 1953-1959, Watanabe and co-workers determined the Ionfzation potentials of some 300 molecules utilizing 7-15 eV photons produced by a hydrogen D.C. capillary discharge and dispersed by a l-meter vacuum monochromator. 41,45 For ionization potential measurements up to approximately $11.5 \mathrm{eV}$, IIF windows were used on the photolonization chambers. At higher energies, windowless chambers. were employed. Because mass analysis was not utilized, the possibility of error due to sample impurities was present. In addition, there was no way to distinguish between Ionization of the parent molecules and processes such as fragmentation and Lonization of dimers, 46 etc. Nevertheless, the threshold value 
obtalned were generally found to be in much better agreement with available spectroscopic ionization potentials than those onsets measured by electron bombardment. It must be noted that these studies still provide the best photolonization Ifterature values for many compounds.

Meanwhile, the use of mass selection in ohotoIonization methods did not appear again unt11 1956-57. 47,48 In these experiments, undispersed ultraviolet radiation was transmitted through LiF windows into the mass spectrometer 1onization chambers. Mass spectra were determined for photolonization products of several compounds and were observed to be considerably less comolex than those obtalned with electron impact.

Experiments combining the advantages of mass spectrometry with improved techniques in the production of monochromatic vacuum ultraviolet radiation were first performed by Hurzeler et.al. 49,50 These investigators used a hydrogen D.C. capillary discharge photon source Isolated from the body of a I-meter Seya-Namioka vacuum monochromator by a LiF window. Dispersed light in the energy range $7.5-11.8 \mathrm{eV}$ was passed through the 1on source of a $60^{\circ}$-sector mass spectrometer. This design permitted the measurement of photolonization efficlency curves for parent and fragment ions of several compounds at an approxinate resolution of $0.05 \mathrm{eV}$. 
The obvious benefits of obtaining monochromatic photon energies above $11.8 \mathrm{eV}$ in mass spectrometric studies encouraged the construction of windowless light source-vacuum ultraviolet monochromator arrangements as early as 1959.51 since that time, photolonization in confunction with mass analysis has been profitably used by many groups. ${ }^{52-64}$ Nevertheless, the basis design of the photolonization mass spectrometer has remalned the same. As mentioned in Section I, instrumental improvements have primarily involved the application of technological advances in ion detection capability and the production of vacuum ultraviolet radiation. Higher lonizing photon fluxes have become avallable as a result of the development of more intense light sources and high resolution vacuum monochromators with increased light throughplit. Ion detection sensitivity has been substantially enhanced by the use of pulse counting techniques. Consequently, photolonization mass spectrometric studies may now he made at optical resolutions typical of many vacuum ultraviolet absorption experiments. Dehmer and Chupka have employed resolutions as high as $0.016 \AA$ or $0.0004 \mathrm{eV}$ at the wavelengths studied. 65 The various aspects of current instrumental design in ohotolonization mass spectrometry will be discussed in a later section. W1th the very high optical resolutions now 
available, considerable attention has recently been given to methods for improving the working resolution of photolonization experiments. It has been aptly demonstrated ${ }^{66-69}$ that true spectroscopic accuracy and precision in the measurement and interpretation of photolon yleld curves is possible only if thermal vibrational and rotational energy effects are largely eliminated or properly taken into account. In room temperature experiments on polyatomic molecules, these effects limit the ultimate resolution to approximately $3 / 2 \mathrm{kT}$ or $0.039 \mathrm{eV}$. As mentioned previously, some success has been achleved in reducing the vibrational and rotational energy spread by a stralghtforward cooling of the sample gas. $65,70-78$ In the case of hydrogen, for example, an effective resolution of at least $0.006 \mathrm{eV}$ can be attained by cooling the gas to liquid nitrogen temperature. Unfortunately, this method is not applicable to easily condensable compounds.

An alternative sampling technique which slgnificantly lessens the detrimental effects of internal thermal energy is the use of supersonic nozzle expansions wherein one takes advantage of the substantial conversion of vibrational, rotational and random translational energy into directed mass motion. The benefits of combining contemporary photolonization mass spectrometric methods with molecular beam samoling were first demonstrated by 
Parr and Taylor in 1973-74. $79-81$ These investigators made high resolution studies of $\mathrm{CO}_{2}$ and $\mathrm{C}_{2} \mathrm{H}_{2}$ using synchrotron radiation as a vacuum ultraviolet light source. Rotational temperatures of the target gas were estimated to be $40 \mathrm{~K}$ for $\mathrm{CO}_{2}$ and $118 \mathrm{~K}$ for acetylene. In addition to producing effective thermal relaxation of the parent molecules, nozzle beams provide unique opportunities to study van der Waals clusters and hydrogen-bonded clusters inasmuch as these species can be formed in relatively large amounts under suitable expansion conditions. As a result, high resolution molecular beam-photolonization mass spectrometric methods are now finding considerable application to studies of various condensable gases and cluster molecules. $82-92$

B. General Considerations

As indicated in previous sections, photolonization mass spectrometry is an established technique for observing and characterizing the various photon absorption channels which result in ionization. Processes such as direct single and multiple ionization and parent ion fragmentation are easily distinguished through the use of mass analysis. Under favorable circumstances, electronic excitations to different ionic spin states as well as 
excitations to optically allowed ionic vibrational levels may be identified in the experimental photoionization efficiency curves. Data pertaining to indirect autoionization and predissoclation processes are also frequently accessible. For even the simplest polyatomic molecules, however, it is obvious that the number of possible transitions increases quickly as a function of excess photon energy above the first Ionization threshold. In order to maximize the amount of useful information in a photolonization mass spectrometric study, it is therefore expedient to perform the experiment at high working resolution and to carefuliy interpret the results by drawling heavily on current understanding of molecular electronic structures and electronic spectra.

1. Photolonization Processes

a. Direct Ionization and the Franck-Cendon Principle

The direct photolonization processes which are most commonly investigated by mass spectrometric methods involve the formation of a singly charged molecular Ion $\mathrm{AB}^{+}\left(1^{-}, \mathrm{v}^{-}, \mathrm{f}^{-}\right)$in electronic state $1^{\prime}$, vibrational state $v^{\prime}$ and rotational state $g^{-}$as a result of the absorption of a photon by the neutral molecule $A B\left(1^{\infty}, v^{\infty}, g^{\infty}\right)$ In its electronic, vibrational and rotattanal states $i^{\prime}, v^{\prime}$ and $j^{\text {N }}$ pespectively. The vast 
majority of these transitions require incident radiation corresponding to the vacuum ultraviolet region (200-2000 $)$. Consequently, the wavelength of the lonfing medium is typlcally much iarger than the linear dimensions of the molecular wave functions. This fact implies that most electronic transitions resulting in the single ionization of a neutral molecule $A B$ may, to a good approximation, be considered to be electric dipole transitions. The probability, $P$, or a transition between $A B\left(1^{\prime}, v^{\cdots}, f^{\cdots}\right)$ and $A B^{+}\left(1^{+}, v^{-}, j^{-}\right)$can then be written in terms of the transition moment integral $93 \overrightarrow{\mathrm{M}}$ as follows:

$$
P \propto \vec{M}^{2}=\left|\left\langle\psi \cdots|\Sigma \vec{P}| \psi^{\circ}\right\rangle\right|^{2}
$$

where $\psi^{\prime \prime}$ and $\psi^{\prime}$ are the inftial and final state eigenfunctions and $\vec{p}$ is the dipole moment operator summed over all electrons and nuclei.

By Introducing the Born-Oppenhe1mer approximation, the eigenfunctions $\Psi(q, Q)$ may be written as a single product of electronic and nuclear functions

$$
\Psi(q, Q)=\Psi_{e}(q, Q) \Psi_{n}(Q)
$$

where $q$ and $Q$ represent the electronic and nuclear coordinates respectively. Similarly, the dipole moment operator can be written as a sum of electrontc and nuclear dependent terms

$$
\Sigma \vec{p}=\Sigma \vec{p}_{e}+\Sigma \vec{p}_{n}
$$


The transition moment integral is then

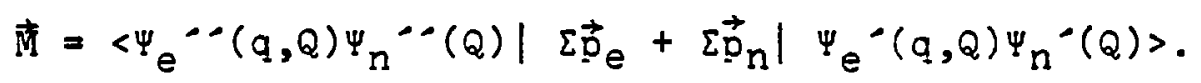

Separating this expression according to the electronic and nuclear parts of $\Sigma \vec{p}$ gives

$$
\begin{aligned}
\vec{M} & =\left\langle\Psi_{n}{ }^{-\prime}(Q) \mid \Psi_{n}-(Q)\right\rangle\left\langle\Psi_{e} e^{-\prime}(q, Q)\left|\sum \vec{D}_{e}\right| \Psi_{e}-(q, Q)\right\rangle \\
& +\left\langle\Psi_{n} \cdots(Q)\left|\sum \vec{p}_{n}\right| \Psi_{n}{ }^{-}(Q)\right\rangle\left\langle\Psi_{e} \cdots(q, Q) \mid \Psi_{e}-(q, Q)\right\rangle .
\end{aligned}
$$

Now $\left\langle\Psi_{e} e^{-(}(q, Q)\right| \Psi_{e} e^{-(q, Q)\rangle=0}$ since the electronic eigerfunctions are mutually orthogonal for an instantaneous nuclear configuration 2.93 Therefore, the transition moment integral becomes

$$
\vec{M}=\left\langle\Psi_{n}{ }^{-}(Q) \mid \Psi_{n}{ }^{-}(Q)\right\rangle\left\langle\Psi_{e}-(q, Q)\left|\sum \vec{D}_{e}\right| \Psi_{e}-(q, Q)\right\rangle .
$$

If vibrational and rotational interactions are neglected, $\Psi_{n}(Q)$ can be separated into a product of vibrational and rotational wavefunctions $\Psi_{V}(Q)$ and $\Psi_{r}(Q)$ respectively. The above equation ( 8 ) may then be written 26 as

$$
\begin{aligned}
& \vec{M}=\left\langle\left(\frac{1}{Q}\right) \Psi_{V^{-}}{ }^{-}(Q) \Psi_{\mathrm{r}}{ }^{\cdots(Q)} \mid\left(\frac{1}{Q}\right) \psi_{\mathrm{V}}{ }^{-(Q)} \Psi_{\mathrm{r}}{ }^{\prime}(Q)\right\rangle \\
& x\left\langle\Psi_{e} e^{-(q, Q)}\left|\sum \vec{p}_{e}\right| \Psi_{e}-(q, Q)\right\rangle \text {. }
\end{aligned}
$$

This expression may be rigorousiy treated by integrating over rotational coordinates. In practice, however, rotational fine structure is unresolved in the photolonization efficiency curves of nost molecules. Neglectirg the rotational motion, the transition moment integral is simplified to

$$
\vec{M} \alpha\left\langle\Psi_{v}{ }^{-\prime}(Q) \mid \Psi_{v}{ }^{-}(Q)\right\rangle\left\langle\Psi_{e} e^{-(q, Q)}\left|\Sigma \vec{p}_{e}\right| \Psi_{e}(q, Q)\right\rangle \text {. }
$$


The first term in Equation (10) is not automatically zero since vibrational functions belonging to different electronic states are often not mutually orthogonal. In general, the second term in Equation (10) does not vary significantly as a function of $Q$. To a good approximation, this integral may be written as $\left\langle\psi_{e}{ }^{\mu}\left(q, Q_{0}\right)\left|\sum \vec{p}_{e}\right| \Psi_{e} \cdot\left(q, Q_{0}\right)\right\rangle$ where $Q_{0}$ corresponds to a position of the nuclel which results in a maximum in the product $\Psi_{\mathrm{V}}{ }^{-} \Psi_{\mathrm{V}}{ }^{\prime}$. Thus, the photolonization transition probability can be separated into factors which depend solely on the electronic motion and nuclear vibrational motion respectively:

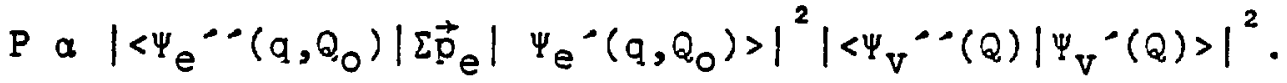

A major result of the foregoing wave mechanical derivation is the well-known Franck-Condon princlple. Given the Born-Oppenhelmer product separation of electronic and nuclear wavefunctions, neglect of rotational motion and the assumption of a constant nonzero electronic transition moment, the relative probabilities of an allowed set of transitions $A B\left(1^{\prime}, v_{n}{ }^{\prime}\right) \rightarrow A B^{+}\left(1^{+}, v_{m}{ }^{\prime}\right)$ are determined by the squares of the respective vibrational overlap integrals:

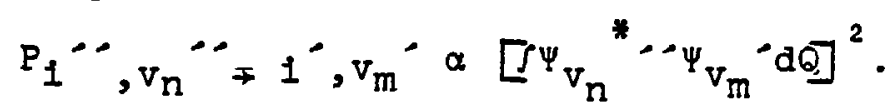

The lonizing transition characterized by the largest square of the vibrational overlap integral (or Franck-Condon factor) for $\mathrm{v}^{\mu}=0$ is of particular interes: 
to photolonization studies. The energy at which this transition occurs is generally called the "vertical Ionization potential." For a given lonic electronic state, this quantity may or may not correspond to the "adiabatic ionization potential" which is defined as the energy at which occurs the transition

$$
A B\left(1^{\infty}, v^{-\infty}=0\right)+A B^{+}\left(1^{+}, v^{\infty}=0\right)+e^{-} \text {. }
$$

Strictly speaking, the term "vertical ionization potential" is in some degree a misnomer. For high vibrational quantum numbers, the correspondence principle indicates that those transitions in which the nuclear configurations are the same in the inftial and final states are most probable. 94 For low vibrational quantum numbers, however, a large vibrational overlap does not always require the relative positions of the nuclei to be the same in the inftial and final states. 95

b. Selection Rules The proper 1dentification and interpretation of observed features in experimental photolonization efficlency curves is facilitated by application of selection rules that indicate whether a glven transition may be expected to be allowed or forbldden. From the discussion In the previous section (II.B.I.a.), it is evident that a photolonization transition is allowed if both integrals of Equation (11) are non-zero. Clearly, the value of each 
Integral is dependent upon the approximations used to define the wavefunctions and operators. Concerning the operators, most photolonization processes within several eV of threshold are well described as one-electron transitions. Therefore, appropriate selection rules may generally be determined by using a one-electron operator in the electronic transition moment term of Equation (11).

\section{Electronic Selection Rules}

Replacing $\Sigma \vec{p}_{e}$ with a dipole moment vector $\vec{p}_{e}$, electronic transitions in the process $A B \rightarrow A B^{+}$are then subject to the general dipole selection rule. That is, an electronic transition between inftial and final states belonging to the same point group is allowed if the product $\Gamma\left(\Psi_{e}{ }^{*}\right) \times \Gamma\left(\vec{p}_{e}\right) \times \Gamma\left(\psi_{e}\right)$ is totally symmetric for at least one component of $\vec{p}_{e} \cdot 93$ If a change of symmetry occurs between the molecule and Ion, the above condition is applied using only those symmetry elements which are common to the two point groups. However, it may be shown that these symmetry requirements do not restrict the number of allowed electronic transitions in photolonization since the final state includes a free electron as well as the molecular Ion. 3 The final state wavefunction $\psi_{e}$ - is properly represented by a positive ion electronic component wavefunction $\psi^{-} e^{+}$and a one-electron continuum wavefunction $\phi_{e} \cdot 26$ Because the continuum wave $\phi_{e}$ may be characterized by a 
mixture of $s, D, d$ or $f$ waves, the electron can always satisfy the angular momentum requirements necessary to make the electronic transition in ment non-zero. This fact gives rise to the rule that all one-electron electronic transitions in photolonization are allowed. This rule remalns true even if a degeneracy exists in one or both states involved in the transition since linear combinations of wavefunctions and dipole components can be found such that $\Gamma\left(\Psi_{e}^{*} \cdots\right) \times \Gamma\left(\vec{p}_{e}\right) \times \Gamma\left(\Psi_{e}\right)$ is totally symmetric with respect to all common symmetry elements.

\section{Spin Selection Rules}

For systems characterlzed by small spin-orbit interaction, the electronic wavefunctions including spin are commoniy separated 93 into the product of orbital and spin functions:

$$
\Psi_{e}=\Psi_{o r b} \Psi_{S} \text {. }
$$

The one-electron electronic transition moment becomes

$$
\left\langle\Psi_{e}{ }^{\cdots \mid}\left|\vec{p}_{e}\right| \Psi_{e}{ }^{\prime}\right\rangle=\left\langle\Psi_{\text {orb }}\left|\vec{p}_{e}\right| \Psi_{o r b}\right\rangle\left\langle\Psi_{s} \cdots \mid \Psi_{s}{ }^{\prime}\right\rangle \text {. }
$$

The dipole moment vector $\vec{p}_{e}$ does not appear in the spin term since it does not operate on spin coordinates. Now, $\left\langle\Psi_{S}{ }^{\prime \prime} \mid \Psi_{S}{ }^{\prime}\right\rangle=0$ for states of different spin due to orthogonality of spin functions corresponding to different spin values. Therefore, a transition is allowed only if total spin, $S$, is conserved between the initial and final states. 
Since the final state in single photolonization involves the removal of an electron with $\operatorname{spin} \mathrm{m}_{\mathrm{S}}= \pm 1 / 2$, the change in multiplicity between neutral molecule and ion must be

$$
\Delta S= \pm 1 \text {. }
$$

This rule indicates, for example, that an fonizing transition from the singlet molecular ground state of a closed-shell molecule produces a doublet Ionic state.

\section{Vibrational Selection Rules}

The electronic and spin selection rules outlined above describe the allowed one-electron electronic transitions in phctolonization. In practice, however, these rules offer little information for interpreting the experimental photolon yleld curves. In contrast, application of dipole vibrational selection ruies can result in a considerable simplification of interpretation for molecules containing symmetry. In an electronically allowed transition, $\left\langle\Psi_{e} e^{\mu \mid \vec{D}_{e}} \mid \Psi_{e}{ }^{\prime}\right\rangle$ is always non-zero. Hence, the vibrational overlap integral $\left\langle\Psi_{v_{n}} "|| \Psi_{v_{m}}\right\rangle$ determines whether or not a transition $A B\left(1^{\infty}, v_{n}{ }^{\infty}\right) \rightarrow A B^{+}\left(1^{+}, v_{m}{ }^{\prime}\right)$ is allowed. If the molecule and ion belong to the same molecular point group, $\left\langle\Psi_{v_{n}}{ }^{\prime \mid} \mid \Psi_{v_{m}}{ }^{\prime}\right\rangle$ is non-zero when the product $\Gamma\left(\Psi_{v_{n}}^{*}{ }^{\prime}\right) \times \Gamma\left(\Psi_{v_{m}}{ }^{\prime}\right)$ is totally symmetric with respect to all polnt group symmetry operations. This condition requires that the viorational levels in the 
Initial and final state have identical symmetry. This result contains significant implications for the analysis of photolonization spectra. Especially when supersonic molecular beam sampling is employed, the preponderance of target molecules in a photolonization experiment exist in the totally symmetric zero-point vibrational level of the ground state. Consequently, only transisions to totally symmetric vibrational levels of the fonic state are allowed. That is, restrictions exist on the number of possible transitions $A B\left(X, v_{n}{ }^{-}\right)+A B^{+}\left(1^{+}, v_{m}{ }^{-}\right)$.

In Ionization from the $\mathrm{v}^{\cdots}=0$ level of the ground state, three types of possible progressions may be distinguished. These types correspond to excitation of totally symmetric ionic vibrational modes, antisymmetric ionic vibrational modes and degenerate ionic vibrational modes respectively. Since the higher vibrational levels of a totally symmetric mode are necessarily also fully symmetric, excltation of a totaliy symmetric vibration occurs in a progression in single quanta. Several such progressions may be observed in a polyatomic molecule, depending on the number of fully symmetric modes. In contrast, the vibrational wavefunctions for antisymmetric modes are totally symmetric for even and antisymmetric for odd vibrational quantum numbers. As a result, transitions to the $v^{0}=2,4,6, \ldots$ levels of these modes are aliowed 
while transitions to the $v^{2}=1,3,5 \ldots$ levels are forbidden. The vibrational selection rules pertaining to excitation of degenerate Ionic vibrational modes are somewhat more complicated.26,93 However, it is sufficlent to point out that weak transitions to the $v^{*}=2,4,6 \ldots$ levels of degenerate modes may occur and transitions to $\mathrm{v}^{*}=3,5 \ldots$ levels may occasionally be very weakly allowed. Transitions to the $v^{\prime}=I$ level of these modes are always strictly forbidden because the first level of a degenerate vibration is never fully symmetric.

The preceding considerations demonstrate that symmetry requirements may result in a substantial simplification of photolonization vibrational spectra. Thus far the discussion has been restricted to vibrational selection rules pertaining to molecules which have the same symmetry in both the ground and ionic state. In addition, it has been Implicitly assumed that both electronic states involved in the transition are non-degenerate. Conditions at variance with the above lead to important complications which deserve brief mention. If ionization results in a final state in which the equilibrium conformation belongs to a different point group than that for the ground state molecule, only the common symmetry elements are considered. That is, a transition is allowed when the product $\Gamma\left(\Psi_{\mathrm{v}_{\mathrm{n}}}{ }^{\prime}\right) \times \Gamma\left(\Psi_{\mathrm{v}_{\mathrm{m}}}{ }^{\prime}\right)$ is totally symmetric with respect to ail 
common symmetry operations. Under these circumstances, fewer restrictions exist on the number of symmetry allowed processes. This relaxation of symmetry constraints contains diagnostic possibilities in terms of molecular structures. Consider, for example, a transition which would normally be forbldden for $v^{\circ}=1$ on the basis of no change in equilibrium symmetry between molecule and ion. If the photoionization vibrational spectrum includes this mode in a progression in single quanta, a change in molecular point group may be inferred. This behavior is observed in the formation of a non-linear ion from a Iinear symmetric triatomic molecule. Much more difficult complications can arise if one or both of the molecular and Ionfc electronic states are degenerate. Those species which possess orbitally degenerate electronic states contain degenerate vibrational modes also. If the coupling between electronic and vibrational motion is negligibly small, vibrational selection rules apply as above. On the other hand, if vibronic interactions become significantly large, the product separation represented by Equation (4) is no longer valid. Complications which arise in photolonization spectra due to vibronic splittings are manifestations of the well known Jahn-Teller ${ }^{96}$ and Renner-Teller $97-98$ effects. 
Rotational Selection Rules

Each vibrational feature in a photoionization efficiency curve contains fine structure which is due to rotational transitions. In practice, this fine structure is rarely resolved. For those few cases in which some resolution has been achieved, rotational selection rules become important. While a detalled examination of these rules is superfluous to the present discussion, excellent investigations of this topic are available elsewhere. 26,93

Since the vast majority of photolonization processes are adequately described in terms of a single step one-electron transition, the selection rules presented above are sufficlent for the analysis of most photoion yleld curves. Features corresponding to multi-electron transitions are possible through configuration interaction and have been observed in certain photoelectron spectra. 3,26 However, features of this type generally occur at excltation energies in excess of $20 \mathrm{eV}$ and therefore are not likely to interfere with normal one-electron photoionization continua near threshold.

$$
\begin{aligned}
& \text { c. Autolonization } \\
& \text { In addition to direct ionization processes, }
\end{aligned}
$$
there exist several indirect two-step photon absorption mechanisms which are extremely important in photoionization 
studies. These mechanlsms involve the production of an intermediate molecular exclted state lying above the first Ionization limit followed by decay into one of four possible channels:

$$
\begin{aligned}
A B+h \nu+A B_{m}^{*} & -A B^{+}+e^{-} & & \text {Autolonization } \\
& +A B_{n}^{*}+h \nu_{m n} & & \text { Fluorescence Emission } \\
& +A+B & & \text { Predissociation } \\
& +A^{+}+B^{-} & & \text {Ion Pair Production }
\end{aligned}
$$

The transition probabilities for the various decay channels vary considerably from one molecular system to another. In general, autolonizing states possess relatively short lifetimes (about 10-14 sec.) with the result that this mechanism is often highly favored. Hence, nearly all photolonization efficlency curves are observed to contain some structure due to this phenomenon. Since the first excitation step represents a resonance process, autoionization manffests itself as peak-like structure on the experimental photolon yleld curves such as would be expected from transitions involving discrete states. In accordance with the Heisenberg uncertainty princlple, the peak widths increase proportionately as the autoionlzing state lifetime decreases. Fluorescence emission, on the other hand, tends to be a relatively improbable decay channel for the intermediate exclted state since this mechanism typically requires $10^{-8}$ seconds or more. Ion-pair formation can occur quite readiz 
where circumstance provides a favorable conflguration of potential surfaces; however, the indirect process which most often competes significantly. With autolonization is predissociation into neutral fragments. As a rule, autolonization features dominate the spectra of small molecules while predissoclation becomes increasingly favored for more complex specles.

The selection rules for autolonization and predissoclation processes are determined by the rules corresponding to each step. Since the inftial transition typically involves a straightforward one-electron excitation due to photon absorption, normal optical selection rules establish the accessible states. The second step in each case represents a radiationless transition. Therefore, it observes the monopole selection rule

$$
\Gamma\left(\Psi_{d}\right)=\Gamma\left(\Psi_{c}\right)
$$

where $\Psi_{d}$ and $\Psi_{c}$ represent the wavefunctions of the discrete intermediate excited state and the continuous final state respectively. If the equilibrium conformations in the two states of the system have common symmetry, $\Psi_{d}$ and $\Psi_{c}$ must have the same symmetry species in order for the autcionization or predissociation step to have non-zero probability. If the two states possess equilibrium orlentations belonging 
to different point groups, elements of symmetry common to both point groups are considered. A discussion of the derivation of these rules from Wentzel's expression for radiationless transition probability 99,100 as well as a detalled treatment of specific selection rules for predissociation may be found elsewhere. 93

It should be noted that a molecule can undergo a transition from the ground state so a bound state lying above the first ionization limit = rough excitation of more than one electron as well as througi excltat:lon of a single non-valence electron. As indicated in the discussion on direct ionization processes, multi-electron exc1tation usualiy requires high energy photon absorption. The formation of the $2 \mathrm{~s} 2 \mathrm{p}^{I_{p}}$ autolonizing state of helium from the (1s) ${ }^{2} I_{S}$ ground state is an example of a two-electron mechanism; ${ }^{101}$ however, a photon energy of $60.0 \mathrm{eV}$ is needed for this transition. At energies appropriate to normal photolonization continua (about 8-20 ev), autoionizing states produced by excltation of non-valence electrons are encountered far more often.

The peak-like features which arise in experimental photolonization efflclency curves as a result of non-valence electron autolonizing transitions can frequently be classifled in terms of smooth progressions along one or more empirically determined series. The orototype for these 
progressions is the Rydberg series of lines which are observed in the absorption spectrun of the ground state hydrogen atom. These lines become more closely spaced with increasing quantum number and merge into the ionization continuum as defined by the Rydberg equation

$$
h v_{n}=I P-\frac{R}{n^{2}}
$$

where IP is the ionization potential of $H$ atom, $R$ is the Rydberg constant, $\mathrm{n}$ is the principal quantum number of the excited electron and $h \nu_{n}$ is the excitation energy of the nth Rydberg state. If a non-valence electron is removed sufficlently far from the lon core of a polyatomic molecule, it experiences very nearly the action of a unit charge central field. Such a system may legitimately be considered more or less hydrogen atom-like. Accordingly, there exists a Rydberg-like series of states whlch can be approximately fitted to the modified hydrogen atom formula given in Equation (1). In this formula, the quantum defect, $\delta$, represents the deviation of the molecular Rydberg series from the $H$ atom case. Each optically allowed quantum state above the zero-point level of the molecular ion ground state represents the lonization limit for an excited electron serles of this type. Further, each series contalns discrete states which lie above the threshold of the first ionization continuum. Each of these bound states may be considered 
to consist of an excited Rydberg electron plus an excited Ion core. Radiationless transitions from these states to the ionization continuum should result in a well-defined progression of peak-like autolonization features in the photoion yield curves. Thus, the analysis of resolvable autolonization structure in photolonization studies principally involves the empirical correlation of peak positions with one or more modified Rydberg formulas.

Radiationless autolonizing transitions from bound molecular Rydberg states occur by the transfer of energy from the excited ion core to the Rydberg electron. While varying amounts of ion core electronic, vibrational and rotational energy may be involved in this transfer, 101 most observed autoionization processes may be interpreted In terms of the interaction of discrete and continuum electronic configurations. The theoretical aspects of this type of autolonization have been extensively investigated by Fano $102-104$ and Mies ${ }^{105}$ using configuration interaction methods. Autoionization by configuration interaction entails the transition of the excited molecular core to a lower electronlc state, with the excess energy carried away by the ejected photoelectron. Since the electron is not restricted to a specific kinetic energy, the resulting Ion may be formed in any final state with a lower energy than that of the Rydber state. According to the 
theoretical treatments of Bardsiey 106 and Smith, 107 the vibrational intensity distribution within a particular electronic state may often be directiy correlated with the Franck-Condon factors connecting the excited autolonizing state with the final state.

When the excited bound state of a molecule consists of an eleztron in a high principal quantum number Rydberg orbital and an ion core with enough vibrational excitation such that the total energy of the system exceeds the ionization limit, vibrational autoionization may occur. The physical picture for this type of autolonization is that the excited molecular ion core undergoes vibrational relaxation whereupon the vibrational energy of the core is converted into enough electronic energy to eject the Ry electron. The vibronic coupling which gives rise to this process corresponds to a breakdown of the BornOppenheimer approximation. The energetics of the vibrational autoionization mechanism has been the subject of several theoretical discussions. ${ }^{108-114}$ An important conclusion from the various models is that the rate of viorationally induced autolonization is expected to lie between the slow process of fluorescence emission and the fast rate of autoionization by configuration interaction. This prediction has been verifled by photoionization experiments on the $\mathrm{H}_{2}$ molecule. $78,115,116$ a second con- 
clusion from the theory is Berry's "propensity rule"110 which states that autolonization processes which involve a minimum change in vibrational quantum number are most probable. Experimentally, the aforementioned $\mathrm{H}_{2}$ photoIonization efficiency curves appear to support this selection rule. In contrast, a recent analysis 117 of the photolon yield curve of No indicates that $\Delta v<-1$ transitions are quite common.

In principle, autoionization by rotationalelectronic interaction is also possible in a manner analogous to vibrationally induced processes. This mechanism has in fact been observed in the very high resolution $\mathrm{H}_{2}$ photolonization measurements of Chupka and. Berkowitz. ${ }^{78,116}$ However, this phenomenon is not expected to be resolved in photolonization efficlency curves taken under normal optical conditions.

2. Photolonization Efficiency Curves.

A photolon yield curve typically contains a record of many superimposed ionization features over a range of excess energy above the first Ionization limit. Once the fundamental conditions for producing specific structure in photolonization efficiency curves are understood, it is possible to translate this record into molecular and ionic state energetic information. A logical method for 
categorizing this information is to consider separately those quantities which can be obtained from (1) threshold data, (2) fine structure data and (3) appropriate thermodynamic cyciles. In addition, there are numerous experimental aspects of data collection and analysis which deserve mention. The special problems pertalning to appearance potential measurements of dissociative processes will be discussed in a separate section.

a. Physical Conditions Leading to Coarse and Fine Structure

For a given chemical system, the fundamental properties of interest in a photoionization measurement are generally the first and higher ionization limits. As indicated previously, photolonization methods are particularly sulted for threshold determinations of this type for two main reasons. First, the avallability of high Intensity light sources and sophisticated vacuum ultraviolet monochromators permits excellent photon energy resolution. Second, the photolonization cross sections for direct ionization channels commonly exhibit a step-like behavior as a function of excess photon energy near threshold. The so-called threshold laws which have been derived from theoretical quantum-mechanical treatments $14-16,18,35$ of the Ionization process are generally used to interpret the observed cross-section energy deperdences. These treat- 
ments suggest that the functional form of the plobability of lonization, $P(E)$, depends principally on the number of electrons, $n$, leaving the lonization site as given by the expression

$$
P(E) \propto k\left(E-E_{T}\right)^{n-1}, E>E_{T}
$$

where $E$ is the lonizing energy, $E_{T}$ is the threshold energy and $k$ is a constant. For direct single photolonization, $n=1$ and Equation (19) becomes

$$
P(E) \propto k\left(E-E_{T}\right)^{0} \text {. }
$$

That is, the lonization efficiency is predicted to behave as a zero-order function of the excess energy, rising abruptly at the onset and remaining constant as $E$ Increases.

It is very important to avold overextending the photolonization threshold law beyond its legitimate scope. As Rosenstock has indicated, ${ }^{1}$ the validity of the law may be restricted to a very narrow energy range above threshold. Also, the range of validity may significantly depend on the system studied. In the absence of complicating factors, experimental evidence suggests that the cross section for a direct photolonization process tends to Increase sharply at onset and fall off slowly at higher energies. This behavior is perhaps best illustrated by the simple photolonization efficlency curve for II 118 as shown In Figure 1. Deviations from a cross-section energy 


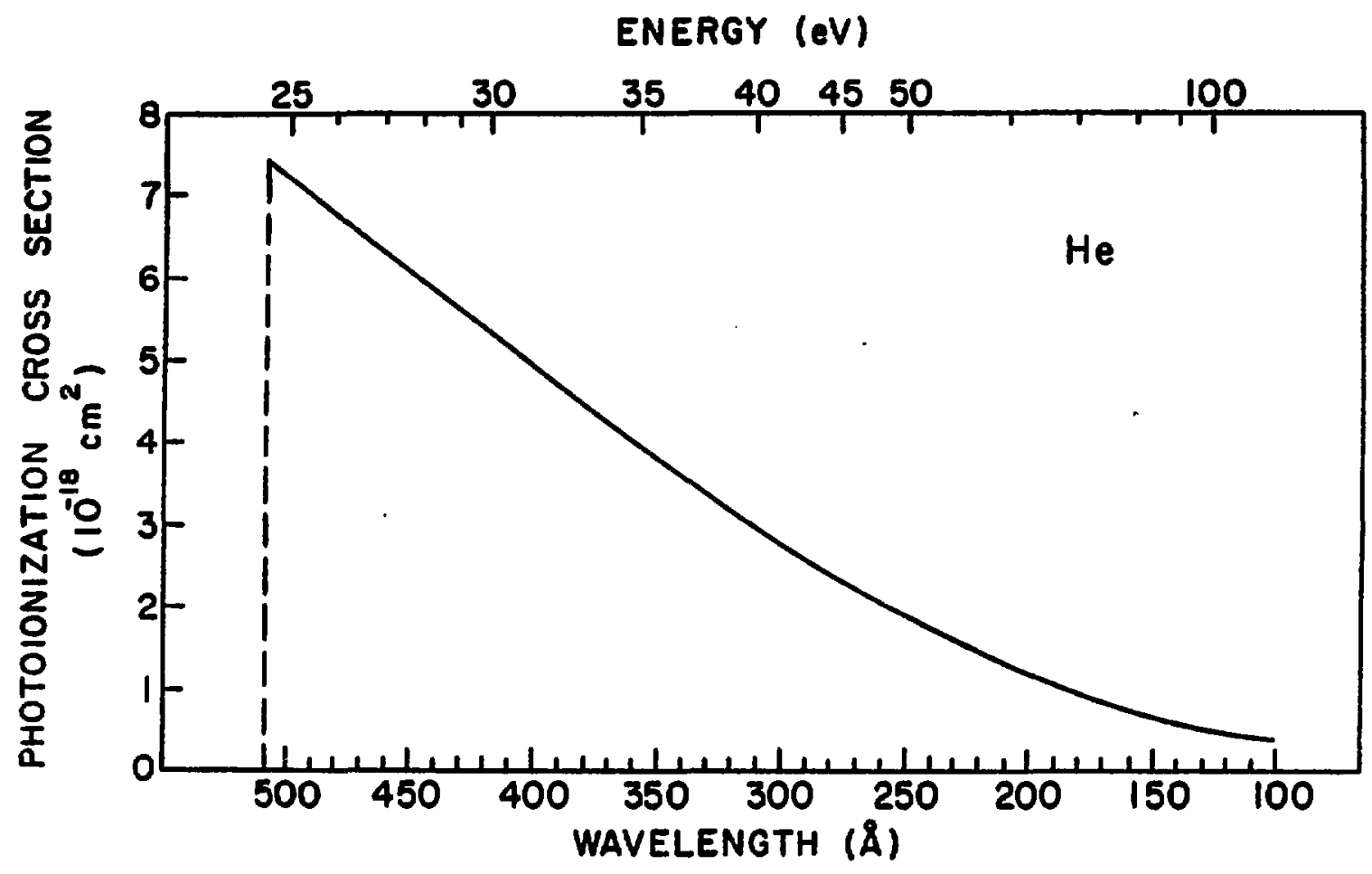

Figure 1. Photolonization Cross Section for Helium (from Ref. I18). 
dependence of this type are not uncommon, however. For example, the photolon yield curves of the alkali atoms exhibit fairly gradual thresholds and large variations at higher energies. In any case, photolonization threshold values are usually well enough defined that near spectroscopic accuracy in the determination of ionization potentials is commonly achieved.

For a polyatomic closed-shell molecule, a large number of superimposed step-like features corresponding to various electronic, spin, vibrational and rotational transitions might reasonably be expected in an ldealized high resolution photolonization efficiency curve. Such steps would represent discrete increases in the photolonization cross section as a function of energy due to the avallability of additional ionization channels. Structure of this type is indeed observed in the photoion yield curves of many molecules. However, for reasons to be discussed later, the appearance of direct lonization mechanisms as essentially discontinuous steps in photoIonization spectra requires favorable conditions with respect to competing indirect processes.

According to the selection rules developed previously, all one-electron electronic transitions are allowed in photolonization. Therefore, the coarse structure 
of an experimental photoionization efficiency curve should in principle contain thresholds corresponding to all energetically accessible excitations from the molecular ground state to the ground and higher electronic states of the 1on. For many molecules, a photon energy scan in the range of about $10 \mathrm{eV}$ above onset reveals one or more higher ionization limits. Transition probabilities for first and higher ionization thresholds typically vary only within a factor of two or three.

Because of resolution limitations, very fine rotational structure is rarely seen in experimental photolon yield curves. Splittings due to spin are also frequently undiscrined. Thus, the preponderance of observed direct Ionization fine structure above threshold arises from transitions between the ground molecular state and available excited Ionic vibrational states. UnravelIing the complex vibrational structure in the spectra of a polyatomic ion is clearly a formidable task. As mentioned previously, this task is significantly simplified in the case of a molecule containing symmetry by the existence of vibrational selection rules in electronic transitions. For example, only eight of the twenty-four normal modes of acetone ion are $A_{1}$ fully symmetric (assuming acetone retains $C_{2 v}$ symmetry upon ionization). Therefore, progressions in 
single quanta are allowed for these vibrations only. Transitions from $v^{\prime \prime}=0$ to the $v^{\prime}=I$ state of the other 16 modes are strictly symmetry forbldden. As a result, the analysis of vibrational structure near the first Ionlzation onset involves the consideration of relatively few excitations.

The relative probabilities of symmetry allowed transitions $A B\left(1^{\cdots}, v_{n}{ }^{\prime}\right) \rightarrow A B^{+}\left(i^{+}, v_{m}{ }^{\prime}\right)$ typically give rise to a distinctive distribution of step helghts In a given photolonization efficlency curve. To a good approximation, these probabilities correlate with corresponding squares of normalized vibrational overlap integrals (Franck-Condon factors). Two physical characteristics are important in determining the value of these integrals: (I) the difference in equilibrium geometry between the molecular and Ionic state and (2) the difference in normal vibrational frequencies. 94 computations using harmonic oscillator wavefunctions indicate that the former factor is generally the larger. The influence of equilibrium geometry change on the shape of photolon yield curves is well illustrated by Rosenstock's adaptation ${ }^{1}$ of Krupenie's calculations, 119 shown in Figure 2. For a small change in equilibrium conformation, a strong $v^{\prime \prime}=0 \rightarrow v^{\prime}=0$ band is expected. In addition, the adiabatic transition is usually accompanied by short and weak 

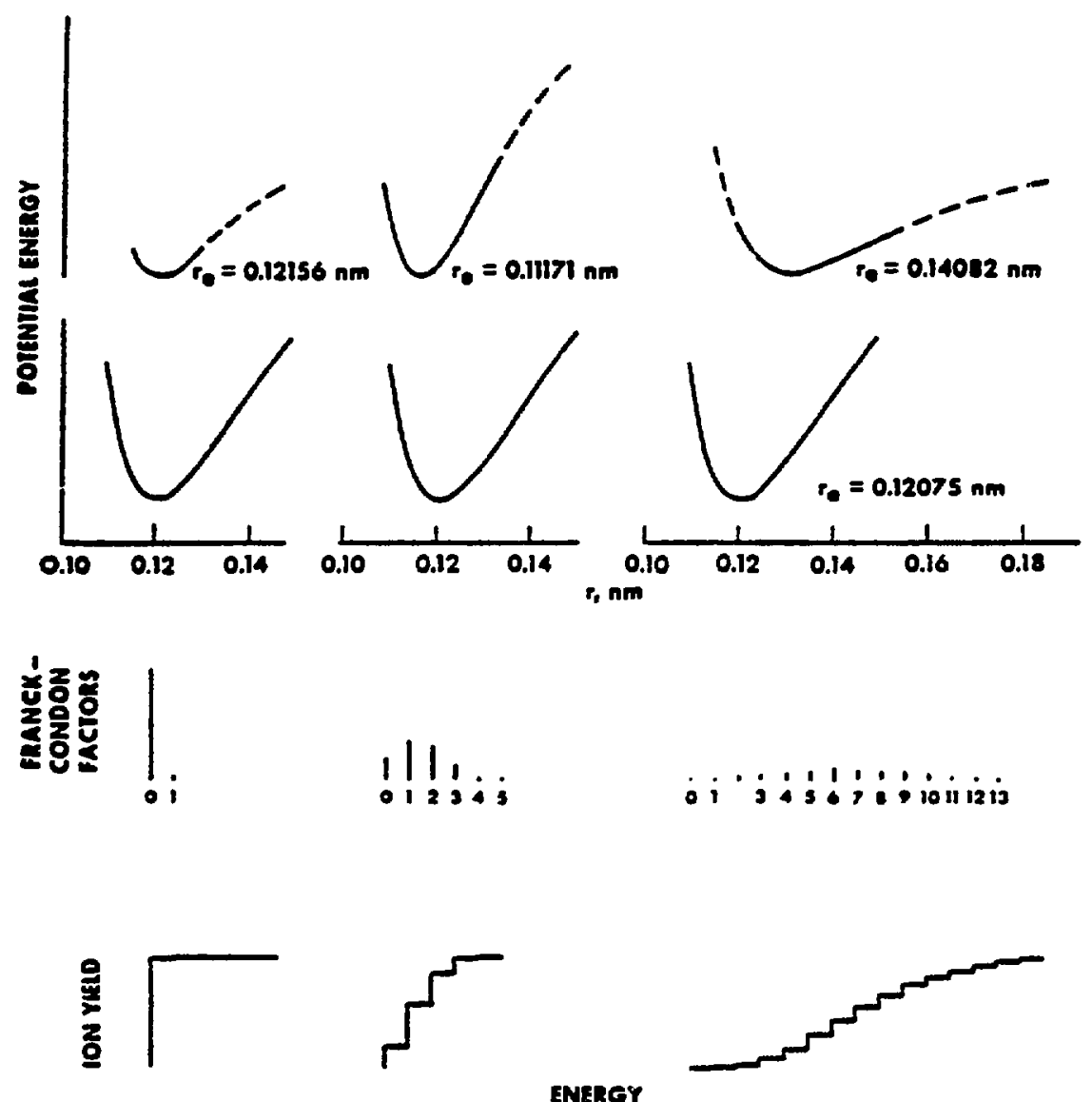

Figure 2:

Calculated Franck-Condon Factors and

Idealized Photolonization Efficiency

- Curves Corresponding to Varlous Changes

$\therefore \quad$ In Bond Length for a Single Oscillator (from Ref. 1). 
vibrational progressions. This behavior generally corresponds to the removal of a non-bonding electron in a system in which the initial and final state symmetries are 1dentical. In contrast, the ejection of a bonding or antibonding electron will likely result in a significant change in equilibrium geometry. In such cases, long vibrational progressions may be seen and the maximum transition probability may occur for $\mathrm{v}^{\mu}=0 \rightarrow \mathrm{v}^{\circ} \neq 0$. The most active modes are typically those which Involve a change in nuclear positions near the site where the ejected electron was primarily localized. For very large changes in potential energy surface, it is not certain that the adiabatic transition will be observed above background.

A change in symmetry between the molecular and lonfc state may be expected to significantly affect the Franck-Condon envelope. For example, the photoion yleld curve of $\mathrm{NH}_{3}$ exhibits 2 weak adiabatic trans1tion 94,120 and a large number of vibrational steps, even though the first ionization limit corresponds to the removal of an essentially non-bonding electron. This distribution of relative transition probabilities apparently results from the change in geometry from oyramidal to planar upon ejection of the electron. Another example is the long viorational progressions which occur in eransi- 
tions between bent and linear triatomic equilibrium configurations, such as for $\mathrm{NO}_{2} \cdot 121$

Two additional points concerning vibrational structure in photolonization efficlency curves deserve mention. First, the comparison of photolonization spectra for two or more isotopes of a given compound can facliftate the assignment of vibrational transitions. Particularly when ionization occurs by the removal of a non-bonding electron, isotopic varlations in the lonlc curves should roughly parallel those seen in the vibrational. spectra of the neutral molecule. Second, if a variation in the spacing of a long progression of vibrational levels is found, it is possible in principle to obtain a measure of the anharmonicity of that particular mode. The vibration of a diatomic molecule provides the most straightforward example of this procedure. For a single oscillator with vibrational frequency $v$, the energy levels $E_{v}$ are given by the expression

$$
E_{v}=h v\left\{\left(v+\frac{1}{2}\right)+x\left(v+\frac{1}{2}\right)^{2}+y\left(v+\frac{1}{2}\right)^{3}+\ldots\right\}
$$

where $\mathrm{v}$ is the riorational quantum number and $\mathrm{x}$ and $\mathrm{y}$ represent first and second order anharmonicity constants respectively. If the linear and quadratic terms only are retained, the vibrational energy level spacing $\Delta E_{V}$ can be written

$$
\Delta E_{v}=h \nu+2 h \times v(v+1)
$$


where $v$ is the vibrational quantum number of the lower energy state of two adjacent levels. That is, $\Delta E_{v}$ should be a linear function of $v$ with a slope of $2 h x v$. Hence, a plot of $\Delta E_{v}$ versus $v$ should yleld the first anharmonicity constant, $x$.

While direct vibrational excitations constitute a major source of fine structure in experimental photolon yleld curves, indirect photon absorption mechanisms often compete significantly. For a given chemical system, the shape of the photolonization efficlency curve can be profoundly affected by the relative rates at which various autolonization and predissociation processes occur. Since configuration interaction induced autolonization usually occurs rapldly, broad peak-like features may be observed when this phenomenon is present. Features of this type occasionally dominate the structure arising from direct ionization continua, especially for small molecules. In contrast, the slower vibrational autolonization processes are manlfested by very narrow peak widths, as required by the uncertalrty princlple. As described previously, peak positions corresponding to a given autolonization series are correlated when possible w1th modified Rydberg equations.

Predissociation mechanisms are not directly observed using ghctoionization mass spectrometric 
techniques. Nevertheless, the competition between predissoclation and autoionization is cruclal to the spectra obtalned by these methods. From arguments relating the density of the osclilator strength for discrete excitations to the variation in absorption cross section, 104 it may be demonstrated theoretically that the average absorption cross section should vary smoothly and continuously through an ionization limit. ${ }^{101}$ Consequently, if the photolonization and photon absorption cross sections for a given molecule are nearly identical, step-like thresholds above the first ionization limit will not be seen in the photolon yleld curve. This suggests that relatively fast nonIonizing absorption mechanisms are necessary in order to obtain step function fine structure due to direct ionization channels. In general, fluorescence emission is much too slow to achieve this effect. However, if oredissociation occurs at a sufficient rate to partially depopulate Rydberg states converging to the various thresholds, discontinuous onsets may be observed. This is frequently the case, especially for larger molecules.

The comolex possibilities for ine structure In photolonization efficlency curves may be illustrated by a few examples. Figure 3 shows the photolonization cross section curve for $\mathrm{Ar}^{+} .73$ Direct ionization occurs to 
ENERGY, eV

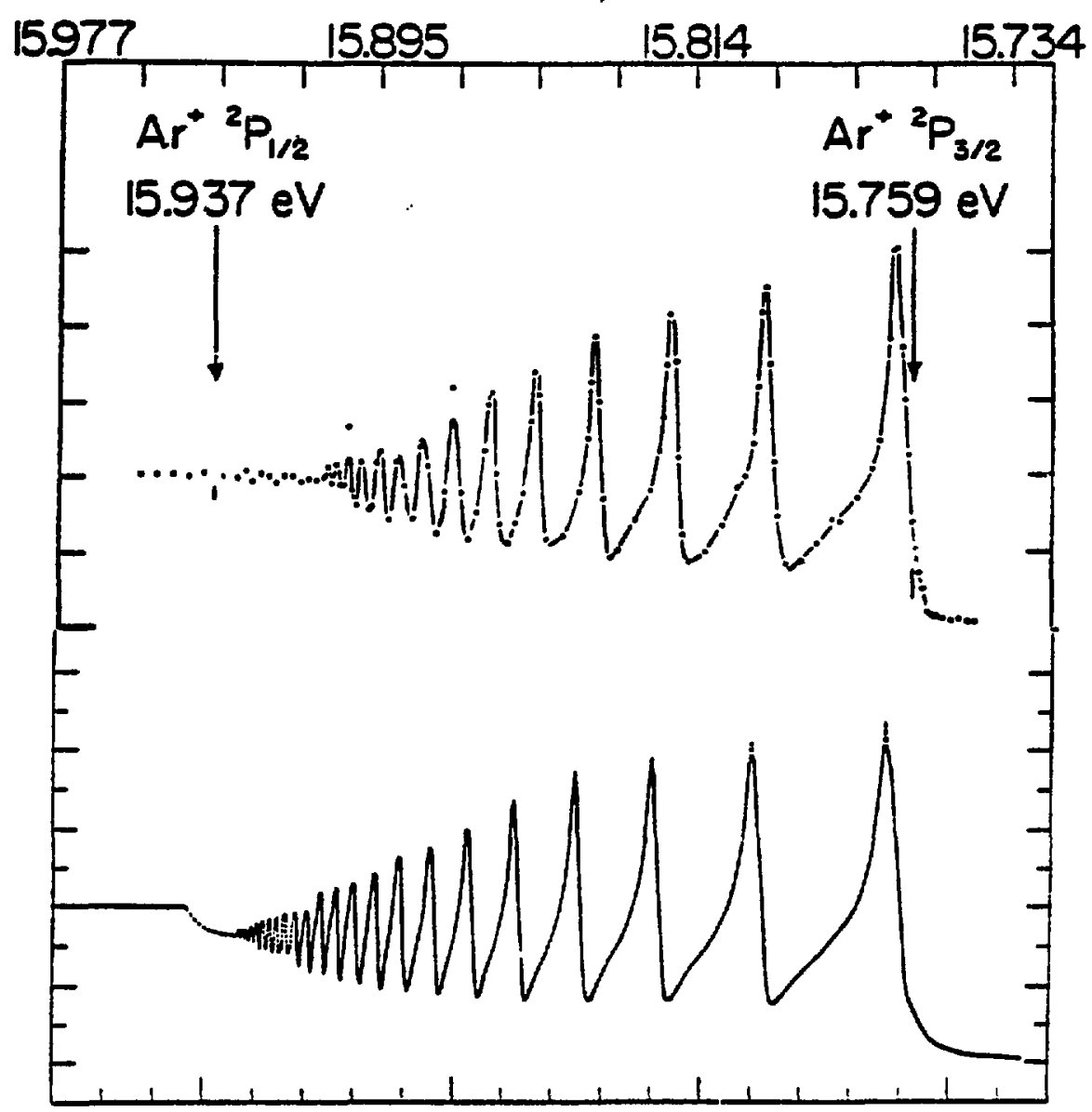

Figure 3. Photoionization Efficiency Curve for Argon (from Ref. 73). 
the ${ }^{2} P_{3 / 2}$ spin state of the ion. The splitting by spinorbit interaction is large enough (about $0.178 \mathrm{eV}$ ) to be easily resolved and the spectrum above threshold is dominated by a well defined electronic autolonization series converging to the upper ${ }^{2} \mathrm{P}_{1 / 2}$ state. Individual peaks are fairly broad as expected for relatively fast configuration interaction induced processes. Perhaps the most interesting feature of the curve is the absence of a discontinuous threshold for the formation of the excited spin state. This behavior results from the fact that almost all photon absorption leads to lonization. In other words, fluorescence emission (the only possible non-1onizing photon absorption mechanism for this system) does not appreciably compete with autolonization.

As shown in Figure 4, the photoionization efficiency curve of $\mathrm{NO}^{89}$ exhibits substantial fine structure above the first Ionization limit. Excitation of successive vibrational levels in the ${ }^{l_{\Sigma}}{ }^{+}$ionic ground state results in a regular staircase structure. Although the initial onset undoubtedly corresponds to the $v^{\prime \prime}=0 \rightarrow v^{*}=0$ transition, a small difference in the equilibrium configurations of the molecule and ion is indicated by the distribution of transition probabilities. Predissociation appears to be the dominant indirect absorption process inasmuch as the threshold of each vibrational level is extremely sharp; 


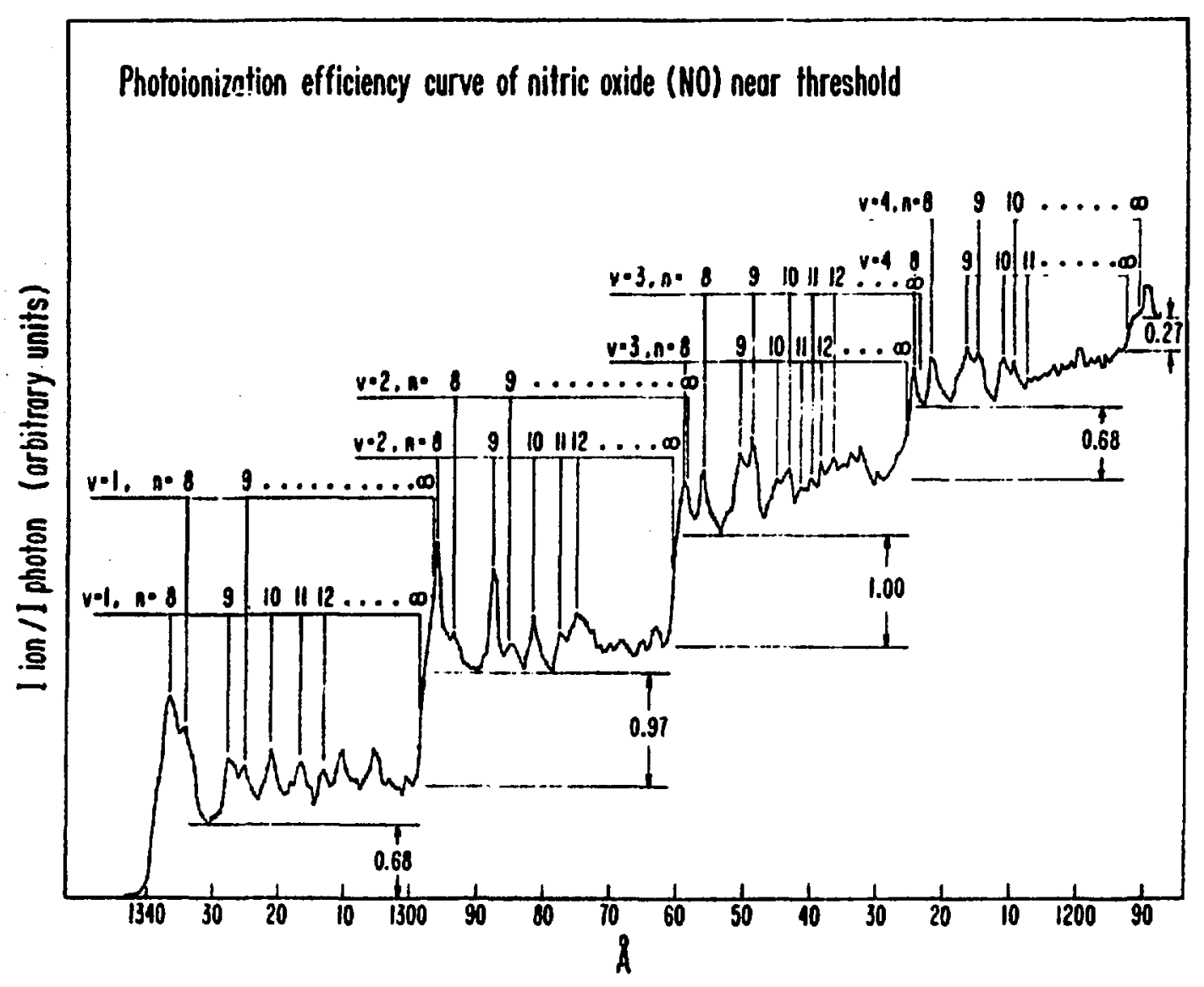


however, weak vibrationally induced autoionization channels produce a defintte structure superimposed on the direct Ionization continua. This structure has very recently been reasslened 117 to a combination of np and nd Rydberg levels. Given this assignment, the individual peaks are quite narrow. A relatively slow rate of vibrational autoionization produces this phenomenon. The photolon yleld curve of $\mathrm{CS}_{2}{ }^{122}$ shown In Figure 5 provides an interesting contrast to the NO data. In this curve, the preponderance of observed autoIonization is presumably due to configuration interaction. These autoionization features are so intense that they obscure the direct photoionization structure throughout most of the spectrum. In fact, the interference is so great that the only transition to an electronically exclted state which is clearly seen is the threshold of the ${ }^{2} \Sigma_{u}^{+}$state at about $14.5 \mathrm{eV}$. By comparison, photoelectron spectroscopy studies 123,124 resolve several electronic states of $\mathrm{CS}_{2}{ }^{+}$with ionization potentials in the energy range 12-20 eV. A signiffcant change in the shape of the potential energy surface between the molecule and Ion is suggested by the very weak inftial onset.

b. Molecular and Ionfc State Energetic Information The primary emphas is of the preceding dis- 


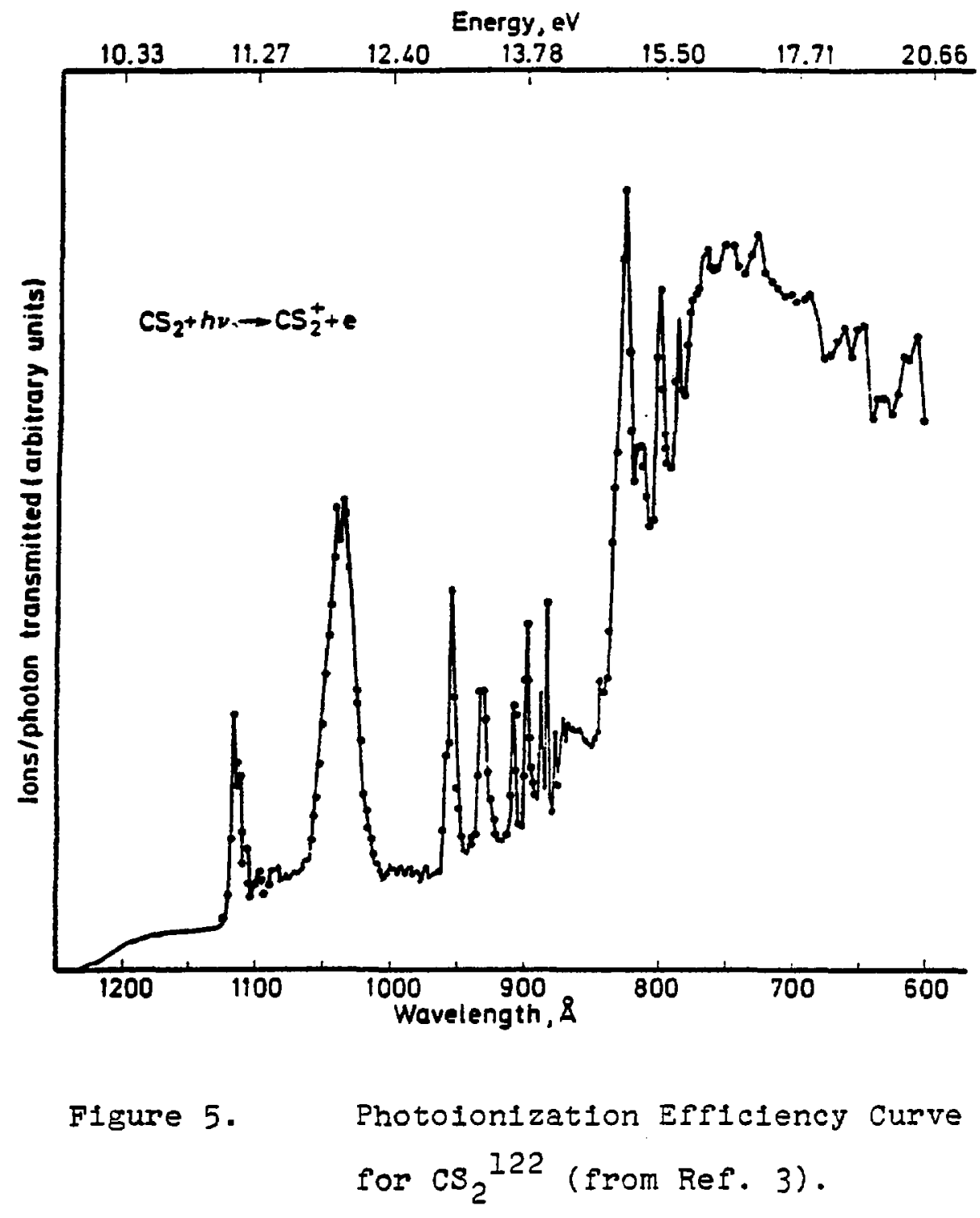


cussion has been to consider how various features in a photolonization efficiency curve can arise from specific sets of conditions relating the molecular and ionic electronic structures. At this point, it is appropriate to briefly summarize the numerous properties pertaining to molecule and ion energetics which can be derlved from an analysis of these features.

From coarse threshold structure, ionization potentials of ground and higher electronic states of an Ion can be determined. The near step function behavior of most onsets in photolonization generally allows considerable accuracy in these measurements. On occasion, Intense autolonization peaks may dominate the direct Ionization continua to such an extent that one or more electronic state thresholds are lost. Under these circumstances, the relatively autolonization-free photoelectron spectroscopy techniques can provide essential complementary data.

In a photoion yleld curve with sultably resolved fine structure, the energy differences between the zero-point level and higher spin, vibrational and rotational levels of a given electronic state may in principle be determined by measuring the separations of appropriate thresholds. For many compounds, ionfc vibrational frequencies and splittings due to spin-ontt 
Interaction can be directly obtained in this manner; however, rotational structure is rarely resolved. In contrast to typical infrared spectra for neutral molecules, the best resolution generaliy occurs for the low frequency vibrations. Information pertaining to the change in the molecular potential energy surface upon fonization is contalned in the Franck-Condon envelope for each vibrational mode. In cases where a long progression of a given Ionic vibration is observed, examination of the viziation in the spacing between successive levels may lead to a measure of the anharmonicity of that mode.

Indirect photon absorption orocesses both influence the direct ionization structure and provide additional information concerning molecular and lonic states. For systems in which predissociation is the predominant indirect mechanism, observed fine structure onsets due to direct ionization will typically be sharp. If autoionization channels are negligible, the helghts of the various vibrational steps can be clearly related to corresponding Franck-Condon factors. On the other hand, if the photoion yield curve contains substantial contribution from autoionization, a correct measure of step helght may be difficult. Autolonization structure which can be correlated with one or more modifled Rydberg series 
can often be useful in that the limits of these series may Identify threshold values which might otherwise be obscure. Furthermore, the quantum defects used to generate these series are interesting insofar as they provide a measure of the deviation of the excited molecular electronic structure from the case of an 1deal central field.

In addition to properties which may be obtained directly from the experimental photolonization efficiency curves, a substantial amount of molecular and Ionfc thermochemical data can be generated by using measured threshold values in appropriate thermodynamic cycles. Th1s procedure may be 1llustrated by a few examples. For direct molecular photoionization, the following relation holds:

$$
\operatorname{IF}\left(A B^{+}\right)=\Delta H_{f}\left(A B^{+}\right)-\Delta H_{f}(A B)
$$

where $\operatorname{IP}\left(\mathrm{AB}^{+}\right)$represents the ionization potential of $A B$ and where $\Delta \mathrm{H}_{f}\left(A B^{+}\right)$and $\Delta \mathrm{H}_{f}(A B)$ refer to the heats of formation of $\mathrm{AB}^{+}$and $A B$ respectively. If $\Delta \mathrm{H}_{f}(A B)$ is known, the determination of IP $\left(A B^{+}\right)$permits the calculaition of $\Delta \mathrm{H}_{f}\left(\mathrm{AB}^{+}\right)$. Another example is the cycle

$$
\begin{gathered}
A B+A B \stackrel{I P_{I}}{\longrightarrow} A^{+}+A B+e^{-} \\
\downarrow-^{-D_{2}} \stackrel{D_{2}^{+}}{\longrightarrow}(A B)_{2}^{+}+e^{-}
\end{gathered}
$$


where $I P_{1}$ and $I P_{2}$ represent the ionization potentials of $A B$ and $(A B)_{2}$ respectively and $D_{2}$ and $D_{2}{ }^{+}$refer to the dissociation energies of the dimer and dimer ion. Since $\mathrm{AB}^{+}$may be formed by either of the two pathways shown, the folluwing relation holds:

$$
I P_{1}=-D_{2}+I P_{2}+D_{2}^{+} \text {. }
$$

In a sultable photoionization mass spectrometric study, $I P_{1}$ and $I P_{2}$ can be directly measured. Therefore, if. $D_{2}$ is known or can be estimated, the dimer ion dissociation energy, $\mathrm{D}_{2}{ }^{+}$, may be calculated. Threshold values for ionic fragmentation processes can also be used in appropriate cycles to derive such quantities as proton affinities, free radical heats of formation, etc. Examples will be given in a later section.

c. Instrumental and Thermal Effects

An ideal measure of photoionization cross section would necessarily involve an infinitely fine resolution determination of the photoion yleld for neutral molecules in their lowest state of internal energy. These conditions could be met only if it were possible to study the sample at $O K$ using an infinitely small photon energy bandwidth. Consequently, the observed data of an actual photolonization experiment may be expected to contain effects due to practical instrumental and thermal limitations. It is important to minimize these effects in order 
to Improve the data collection. Properly taking the remaining limitations into account is essential for careful data analysis.

Instrumental factors which may influence the shape of experimental photolonization efficiency curves include the transmission efficiency of the ion optics, the collection efficiency of the Ion detector, the monochromator entrance and exit slit widths, the output characteristics of the light source, and optical deficiencles resulting in non-homogeneous Ilght and photoelectron production. The variation of ion transmission probability with excess photon energy above threshold is generally negliglble over the energy range corresponding to individual photoion yield curve features. In addition, for experimental designs incorporating large ion accelerating voltages, the fon detector collection efficiency is virtualiy constant as a function of excess energy. Therefore, these factors are usually unlmportant 67 and are not considered further.

A major systematic instrumental Iimitation in photolonization experiments is the unavoldable energy spread of dispersed radiation from continuum light sources. This spread introduces an uncertainty in the measurement of photoionization thresholds which can be minimized but not entirely eliminated. This effect generally occurs in the following manner. The range of the energy band which 
a given lamp-monochromator system passes is princlpally determined by the silt widths employed on the monochromator entrance and exit arms. The variation in photon intensity over such a band, $E_{0} \pm \delta$, typically approximates a triangular or trapezoldal function centered on the nominal photon energy $E_{0}$. The convolution of a slit function of this type with a nearly step-like direct Ionization threshold may be expected to produce a sigmoldal feature with the inflection polnt theoretically corresponding to the ionization onset. 67 The exact location of the threshold naturally becomes less certain as the s-shaped structure becomes broader. To the extent that photon intensity can be sacrificed, narrower slits can be used to establish more precise onsets. However, this procedure is finaliy limited by the ultimate resolution of the monochromator optics.

Given the highly refined current technology for producing intense, nearly monochromatic photon beams, uncertainties in the measurement of photoionization onsets can be made very small. A far more serlous problem is spurious photoion yield curve features which can arise from varlous sources. For example, Nicholson has shown 125 that false structure in photolonization spectra can result from the use of a discrete-line light source. These features were seer fn the vidutty of the very strong Lyman $a$ 
$(1215.7 \AA)$ and Lyman $B(1025.7 \AA)$ lines in experiments using the many-ine pseudo-continuum produced by a hydrogen discharge lamp. Now, phenomena of this type should occur independently of the system studied. Therefore, it is possible to identify such artifacts by comparing the photolonization efflclency curves for several specles. Other sources of spurious structure are lonlzing photoelectrons and non-homogeneous light orlginating from defects in instrumental design. Large numbers of photoelectrons can be released from a metal surface which intersects the ultraviolet photon beam. If these garticles are accelerated by a sufficient potential inside the apparatus, unwanted lonization may occur. This effect can be minimized by designing the Instrument so as to prevent as much as possible the dispersed light from hitting metal surfaces near the 1onlzation region. Collectors can be installed to attract most of the stray particles which are formed. Photoelectrons which experlence substantial acceleration into the sampling area should produce ionization even if the photon energy lies well below the first ionlzation limit of the system. Moreover, since cross sections for electron impact lonlzation are generally much larger than those for photolonization, artifacts arising from a process of this type are Itkely to be iarge. Consequently, the absence or 
photolon signal below the true inftial onset is a good indication that the contribution to a photolonization efficlency curve from photoelectron bombardment is small. In contrast, the deleterious effects of non-homogeneous light are likely to be falrly subtle. Dispersed radiation far removed from the nominal monochromatic photon energy can be transmitted to the ionization region as a result of ghosts, stray light and higher order reflections from the diffraction grating. Spurlous reflections inside the monochromator body are also possible. The last effect is typically eliminated by removing unnecessary reflecting surfaces and by painting the inside of the monochromator flat black. Higher order grating reflections, on the other hand, can be dealt with by using a light source with negligibly low photon intensity at integral multiples of the desired energy $E_{0}$. If sultable filters exist, these can also be employed. Ghosts and stray light arise from imperfections in the grating which are periodic and nonperiodic respectively. 126 It is quite difficult to determine precisely the extent to which a "monochromatic" photon beam may contain impurity light due to these imperfections; however, intense artificlal lines or severe levels of scattered light should be obvious upon comparison with standard spectra. Needless to say, it is wise to

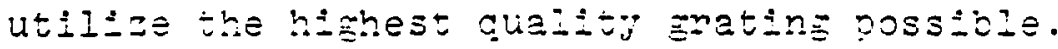


In previous sections, brief mention has been made of the Importance of the average internal thermal. energy of the sample in limiting the working resolution of a photolonization experiment. Specifically, if a significant number of neutral target molecules exist in excited vibrational and rotational states, the photoion yleld curve exhiblts "hot bands," threshold talling and loss of Ine structure definition. In the present context, "hot bands" refer to those features in a photolonization efflciency curve which arise from allowed transitions from excited vibrational levels of the molecular ground state. These features will occur at lower photon energies than those corresponding to direct ionization onsets from $\mathrm{v}^{\cdots}=0$. Structure of this type is particularly troublesome ior systems in which the $\mathrm{v}^{\prime \prime}=0 \rightarrow \mathrm{v}^{*}=0$ transition is weak. In such cases, the true adiabatic ionization potential may correspond to a poorly defined step well above the first appearance of photolons. Dibeler and co-workers have clearly demonstrated 75 the extent to which hot bands affect ionization thresholds for diatomic halogen molecules at room temperature. Since the energy separation of rotational states is often smalier than typical optical resolutions, Individual rotational "hot bands" are not commonly observed. Instead, the rotational energy of the sample approximates a Boltzmann distrioution with an average energy of $3 / 2 \mathrm{~km}$ 
(about $0.039 \mathrm{eV}$ at 298K) for polyatomic specles. This rotational envelope causes talling of the initial onset and an overall loss of ultimate resolution.

It is clearly advantageous to maximize the effective resolution of photolonization spectra by sampling molecules with low internal temperatures. As mentioned previously, the two established techniaues for doing this are (1) stralghtforward cooling of noncondensable gases and (2) supersonic nozzle beam sampling. The latter method w1ll be considered in detall in section II.C.

3. Appearance Potential Measurements of Fragmentation Processes

At sufficiently high photon energies, the photolonization mass spectrum of a polyatomic molecule typically includes several fragmentation products in addition to the parent 1on. These fragments can arise from such processes as direct dissociation to an fon pair or decomposition of the parent ion. The latter mechanism is observed most often. Experimental detection of this mechanism depends on the rate at which dissociation occurs. Fragment ion signals are seen directly only if decomposition takes place in the ionizer. On the other hand, fragment Ions which are produced after mass analysis contribute to 
the parent ion peak while dissoclation products which are formed in the acceleration and magnetic field regions are not observed at all. Those ions which decompose prior to mass analysis but after acceleration give rise to so-called "metastable ion" signals. A fragment ion from a metastable decomposition is detected at an apparent mass position of $\mathrm{m}^{*}$ as given by the expression

$$
\mathrm{m}^{*}=\frac{\left(\mathrm{m}_{\mathrm{f}}\right)^{2}}{\mathrm{~m}_{\mathrm{p}}}
$$

where $m_{f}$ and $m_{p}$ are the masses of the fragment ion and parent ion respectively. Since metasiabie ion peaks generally occur at non-integral mass values and are usually much broader than normal mass spectral features, transitions of this type are easily ldentified.

The fragmentation processes of very small polyatomic lons can be successfully described quantitatively in terms of theoretically and experimentally determined details of potential surfaces. This approach becomes Impractical for larger lons, however. For comolex systems, statistical theories provide a more accessible means of treating dissociation mechanisms. The most widely applied method in this regard is the guasi-equilibrium theory developed by Rosenstock and co-workers. 127-130 This theory is based upon a number of important assumptions. First, the time required for decomposition of the parent 
Ion is considered to be long with respect to the ionization and inftial internal excitation step. Second, dissociation is judged to be slow enough that the excitation energy in the reactant ion has time to be redistributed over all degrees of freedom. Finally, fragmentation is assumed to occur along competitive and sometimes consecutive unimolecular reaction paths where each decomposition process may be described in terms of a separate reaction coordinate wth an appropriate activation energy and activated complex. The first two assumptions are generally consistent with experimental observations ${ }^{131}$ and allow the ionic dissoclation mechanisms to be considered independentiy of the Initial excitation. In view of the low operating sample pressures typically employed in mass spectrometers, few ions expertence collisions with other molecules or ions before extraction from the ionizer. Thus, in effect, ionic fragmentation takes place in an isolated system at internal equilibrium. The final assumption represents a model which is essentialiy common to the absolute rate theory of Eyring, et. al. 132 as well as RRKM theory. ${ }^{133,134}$ Given the above assumptions, the microcanonical quasi-equilibrium theory rate expression may be written as given by Wahrhaftig 130

$$
k_{1}(E)=\frac{\sigma}{h} \frac{W_{1} \neq\left(E-\varepsilon_{1}\right)}{\rho(E)}
$$


where $k_{1}(E)$ is the reaction rate along reaction path $i$ with activation energy $\varepsilon_{1}$ as a function of the reactant Ion Internal energy $E, \sigma$ is the symmetry factor representing the number of 1dentical paths for the decomposition, $h$ is Planck's constant, $W_{1} \neq\left(E-\varepsilon_{1}\right)$ is the number of states of the activated complex lying between $\varepsilon_{1}$ and $E$, and $\rho(E)$ is the density of states for the parent ion. It must be briefly noted that other approaches to unimolecular : onic fragmentation theory have been proposed as well. $130,135,136$

\section{The quantities which are of primary}

Interest in a photolonization mass spectrometric study of dissoclative processes are the observed onsets of lonic fragmentation, commonly called appearance potentials. In particular, it is useful to obtain a measure of these thresholds for molecules in minimum internal energy states. The utility of direct ionization potentials in suitable thermodynamic cycles has been demonstrated previously. In a similar manner, appearance potentials can be used to deduce substantial amounts of thermochemical data. For Instance, the reaction

$$
A B+h \nu+A B^{+}+A^{+}+B+e^{-}
$$

may be characterlzed by the following thermochemical relations

$$
A P\left(A^{+}\right)=\Delta H_{f}\left(A^{+}\right)+\Delta H_{f}(B)-\Delta H_{f}(A B)
$$




$$
A P\left(A^{+}\right)=I P(A)-D(A B)
$$

where $A P\left(A^{+}\right)$is the appearance potential of the $A^{+}$fragment, IP(A) represents the ionization potential of the free radical $A, D(A B)$ is the bond dissociation energy of $A-B$ and $\Delta H_{f}\left(A^{+}\right), \Delta H_{f}(B)$ and $\Delta H_{f}(A B)$ represent the heats of formation of $A^{+}, B$ and $A B$ respectively. Frequently, properties such as $D(A B)$ and $\Delta H_{f}(A B)$ are known from other work. Thus, experimentally determined appearance potentials may permit the calculation of such relatively inaccessible thermodynamic values as $\Delta \mathrm{H}_{f}(B)$ or IP(A). Another example is provided by the cycle

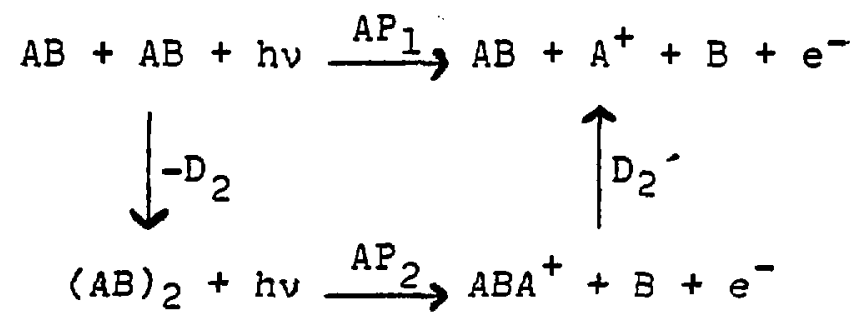

where $A P_{1}$ and $A P_{2}$ are the appearance potentials of $\mathrm{A}^{+}$and $\mathrm{ABA}^{+}$respectively and $\mathrm{D}_{2}$ and $\mathrm{D}_{2}$ ' represent the bond dissoclation energies of $(A B-A B)$ and $\left(A B-A^{+}\right)$. The two mechanisms giving rise to the formation of $\mathrm{A}^{+}$suggest the following relation:

$$
A P_{1}=-D_{2}+A P_{2}+D_{2} \cdot
$$

This relation is very similar to Equation (25). If $D_{2}$ is known or can be estimated, the direct measurement of $\mathrm{AP}_{1}$ and $\mathrm{AP}_{2}$ allows the calculation of the $\left(\mathrm{AB}-\mathrm{A}^{+}\right)$dissociation enersy. Ncreover, subtraction of Equation (32) from 
Equation (25) gives the interesting expression:

$$
\left(I P_{1}-I P_{2}\right)-\left(A P_{1}-A P_{2}\right)=D_{2}^{+}-D_{2} \cdot \text {. }
$$

This equation provides some measure of the influence of the $B$ substituent on the dimer ion bond. In favorable chemical systems, incorporation of appearance potential determinations into other suitable thermodynamic cycles can lead to calculations of solvation energies, proton affinities, electron affinities, etc.

In addition to their direct importance In thermochemical calculations, appearance potentiais may be used in determining ion-molecule reaction mechanisms and internal energies of reactive ions. ${ }^{137}$ The effect of 1sotopic substitution on the relative cross sections for fragment lons is also interesting. In contrast to the typlcally very small differences observed in the lonization potentials of 1sotopic parent specles, isotope effects In appearance potentials can be fairly large. If the appropriate force flelds are known, these effects can be related to the zero-point energy differences of the various constituents in the lonization and fragmentation processes. A particularly instructive study in this regard is the photoionization mass spectrometric experiment on $\mathrm{H}_{2} \mathrm{CO}$, HDCO and $D_{2} C O$ performed by Guyon, Chupka and Berkowitz. 138 Using the threshold law for direct photoionization processes and the quasi-equilibrium theory 
rate expression given in Equation (27), it is possible to derive a theoretical expression for the ionization efficlency of a primary fragment ion. ${ }^{131}$ For the purpose of this discussion, it is sufficient to point out that this expression predicts a falrly complicated relationship between the lonization efficlency and the energy of the Ionlzing medium. This prediction is generally borne out In actual appearance potential measurements. For the most part, fragment ion yleld curves are observed to rise gradually from threshold and rarely exhibit well differentiated fine structure features.

The determination of meaningful appearance potential values from experimental curves is a non-trivial task. Aside from various difficulties associated with the Instrumental sensitivities, at least five major factors have been identified 139 which can cause fragmentation thresholds to appear at higher photon energles than the proper thermochemical onsets. It is useful to restate these problems in some detail inasmuch as they illustrate many important physical aspects of decomposition processes.

(1) According to the quast-equilibrium theory, the rate of lonic dissociation is a function of the largely randomized total internal energy content of the parent 1on. It follows then that the thermochemical threshold for fragmentation corresponas to a given minimum 
excitation energy. However, it may be possible that the transition probability for forming the reactant ion at the appropriate internal energy is very small. Under these clrcumstances, the onset of dissociation will be observed at the lonizing energy necessary to excite the next highest accessible state in the parent ion. Since the Franck-Condon envelope is often narrow in the electronic ground state of the ion, this would frequently be the case if direct $A B\left(X, v^{-\infty}, j^{-\prime}\right)+A B^{+}\left(A, v^{-} ; f^{-}\right)$channels were the only means of populating higher lonic states. Fortunately, autoionization and multi-electron excitation mechanisms as well as transitions to higher electronic states are generally effective in providing the necessary thermal energy range. The possible occurrence of this problem can typlcally be inferred from the photolonization efflclency curve or photoelectron spectrum of the parent species.

(2) In reactions where transitions from exclted molecular or ionic electronic states are required in order for the ground state internal energy content of the ion to reach thermochemical fragmentation thresholds, selection rules may exist which nominally forbld the necessary transition. Because of the relatively long time perlods available for observable dissociation $\left(10^{-4}-10^{-5}\right.$ seconds), these rules rarely 
restrict the possibilities for decomposition, however. In other words, intersystem crossings generally can occur.

(3). If all reaction coordinates for a particular fragmentation pro ess contain potential barrlers, $E_{1}{ }^{*}$, the experimental appearance potential will be displaced from the thermochemlcal onset by the minlmum barrler height, $E_{m i n}^{*}$. In other words, Equation (29) becomes

$$
A P\left(A^{+}\right)=\Delta H_{f}\left(A^{+}\right)-\Delta H_{f}(B)-\Delta H_{f}(A B)+E_{\text {min }}^{*} .
$$

Experimental evidence indicates that $E^{*} \mathrm{~min}$ may range from zero to nearly one electron volt. 1,140 wh1le there are apparently no strict rules, $\mathrm{E}^{*}$ min is often very small for decompositions involving single bond ruptures. On the other hand, large barrler helghts are common for reactions involving rearrangement of bonds. Attemots have been made to develop a procedure whereby $E^{*} \mathrm{~min}$ may be deduced from the kinetic energy release in the fragmentation process. The various approaches which have been used are discussed by Rosenstock. ${ }^{1}$

(4) From the quasi-equilibrium rate expression, Equition (27), the minimum rate for dissociation may be written as

$$
k_{1, \min }=\frac{\sigma}{h \rho\left(\varepsilon_{i}\right)}
$$

where $\varepsilon_{1}$ represents the activation energy for the 
reaction. In order for a decomposition process to be readily observed in a typical mass spectrometer, a rate constant in the range of $10^{5}-10^{7} \mathrm{sec}^{-1}$ is necessary. If $k_{1, \text { min }}$ falls well below this range, fragment ion signals may not be seen until a significant amount of additional internal energy is deposited in the reactant 1on. This excess energy is commonly known as the kinetic shift. 69 In general, dissociation processes Which exhibit this effect may be identified by the occurrence of strong metastable peaks. Since lons of this type arise from slower decompositions than those which take place in the 1onizer, comparison of fragment Ion and "metastable 1on" appearance potentials may be expected to yleld at least part of the kinetic shift. While no certain experimental method exists as yet for measuring the remaining part of this shift, a relationship between this quantity and the kinetic energy release in metastable decompositions has been suggested.141,142 Ion-trapping techniques represent a potentially useful means of determining very nearly the full kinetic shift. Gordon and Reid have employed this method 143 to investigate the excess energies required for a few reactions. These measurements are in falr agreement with Vestal's quasi-equilibrium theory calculations ${ }^{13 I}$ which estimate the kinetic shift for a 
number of typical fragmentation mechanisms. Vestal's results indicate that the additional energy needed for a reaction rate of $10^{5}-10^{6} \mathrm{sec}^{-1}$ is often negligible. In contrast, kinetic shifts of up to $2 \mathrm{eV}$ are predicted for such processes as the loss of $\mathrm{H}$ atom from benzene and toluene lons.

(5) For many systems, a large number of competitive and consecutive ionic decomposition processes may occur within a few $\mathrm{eV}$ of the first lonization limit. Under these clrcumstances, it is often difficult to see many of the higher energy channels at the appropriate thermochemical thresholds. If reaction competition is strong, a less favored mechanism generally requires substantial energy in excess of the activation energy in order to produce detectable fragmentation. That is, large kinetic shifts are observed for these dissoclations. Moreover, the first decomposition in a consecutive process does not produce daughter ions and neutral fragments in specific energy states. Rather, these reaction products are typically formed with a wide distribution of excltation energy. As a result, appearance potential curves corresponding to successive fragmentation mechanisms exhibit poorly defined onsets, rising almost asymptotically from backsround. 
In contrast to the various factors which displace appearance potentials to higher energies, the distribution of excess internal energy in the sample molecules prior to ionization serves to lower the observed onsets. Since the rate of dissociation may be considered to be largely independent of particular excitation mechanisms (according to quasi-equilibrium theory), virtually all of the initial thermal energy In a parent molecule should be effective in ionic decomposition processes. Experimental fragment ion curves may then be expected to contain a low energy "tail" below the proper (OK) thermochemical threshold. In practice, this effect often cancels some of the errors introduced by the five problems mentioned above. The influence of sample thermal energy on appearance potential measurements has been carefully investigated.66-69 One empirical method of correcting for internal energy effects requires data collection at two or more temperatures followed by comparison of threshold differences with changes in internaliy stored energy calculated from heat capacity data. ${ }^{144}$ An alternative method is described by Chupka. 69 Once again, it should be noted that substantial decreases in the thermal energy of the molecules can be achieved by sampling from a supersonic nozzle expansion. This 
technique will greatly diminish, but not entirely eliminate, the shift in onsets.

In summation, many processes pertaining to the dissociation of exclted molecular ions may be studied using photolonization mass spectrometric methods. Appearance potentials, in particular, provide an important measure of the energetics of ionic decompositions. These values are perhaps most useful in determining various thermochemical properties of molecules, fons and free radicals. However, it is clear that a careful interpretation of experimental appearance potential data requires due consideration of numerous possible sources of error.

\section{Supersonic Molecular Beam Sampling}

The first recorded attempt to generate large numbers of molecules in straight line trajectories appears to be Dunoyer's sodtum beam work in 1911.145 Since that time, molecular beam techniques have been widely employed in experimental studies of the properties and behavior of atoms and molecules. Effusive or oven beam methods and supersonic nozzle beams are two particularly useful techniques which have been developed.

Beginning with Otto Stern's work in 1919, much of the attention of early investigators was given to the design and utilization of effusive beams. These 
techniques have consistently been found to be appropriate to various applications and numerous detailed reviews of these methods and results are avallable.146-152 Briefly stated, beam production systems of this type generally consist of a linear manifold of three chambers which communicate only through small apertures or slits. The oven or source chamber contains a gas at a temperature roughly defined by the temperature of the walls. If the pressure in this enclosure is maintained low enough that the mean free path of the gas particles exceeds the dlameter or width of the opening, the molecules escape freely through the aperture into the second cavity. This chamber typically serves to collimate the effusive flow so that the third cavity recelves a well defined beam for investigation or detection. In order to sustain the effusive beam, it is necessary to evacuate the second and third enclosures with suitable vacuum pumps. This assures that the mean free paths of the particles in the beam and in the background gas are longer than the dimensions of the apparatus. In effect, effusive beam methods establish a sampling regime which is characterized by a relatively collision-free unidirectional flow of molecules.

For all their advantages, oven beam techniques suffer from practicai limitations in intensity and 
energy. Intensity restrictions exist because of the mean free path considerations mentioned above. Thus, equivalent effusive beam pressures rarely exceed $10^{-6}-10^{-5}$ torr under typical vacuum conditions. Beam energy Iimitations arise from the lack of gas box construction materials able to withstand temperatures In excess of $3000 \mathrm{~K}$. Since source gas temperatures above this quantity are not feasible, the maximum attalnable average translational energy of molecules In a thermal oven beam corresponds to $2 \mathrm{kT}$ or about $0.5 \mathrm{eV}$. These intensity and energy problems substantially inhibit the applicability of effusive beam methods in experiments having inherently low signal-tonolse ratios and in studies of moderate energy collision processes.

The desire to increase beam intensities has encouraged the development of alternative'beam sources. One moderately successful technique consists of replaclng the single source chamber orffice with an array of parallel channels. This method was apparently first employed by Zacharias in 1953.148 Significant enhancement of beam intensities can be achleved using multichannel arrays, especially at lower net pumping speeds in the collimating chamber. 153 However, far more intense moiecular beams can be produced by substantially 
Increasing the source chamber pressure so that the conventional effusive flow fleld is transformed into a supersonic jet. An additional beam defining aperture is typicaj.Iy placed in the jet in order to meet the increased pumping requirements. This instrumental approach represents the basis of nozzle beam technology.

Supersonic molecular beams developed quite slowly in comparison to oven beam methods. The earliest supersonic jet experiments appear to have been performed by Johnson 154,155 in 1926-27. In these studies, a mercury atom beam was operated at source pressures such that the particle mean free path was much smalier than the narrowest dimension of the exit aperture. Cryogenic pumping was employed to handle the increased throughput. Consequentiy, an additional beam defining element and pumping chamber were not needed. Maximum intensities were observed to be in excess of 100 times larger than those expected for a conventional effusive beam. Another interesting early paper is Rodebush's review of "molecular rays" published in 1931.156 In this articis, a beam defining design is suggested which bears a striking resemblance to many sonic nozzle - conical skimmer arrangements used today. 
It remalned for Kantrowitz and Grey ${ }^{157}$ to reassert the powerful possibilities of supersonic nozzle beam techniques. The instrumental scheme proposed by these authors in 1951 included a converging-diverging (or Laval) source chamber nozzle and a conical skimmer serving as the entrance orlfice of the collimating cavity. A theoretical analysis anticipated several advantages to this design, including large increases in beam intensities. These predictjons were partially verified in the experimental companion paper of Kistiakowsky and Silchter ${ }^{158}$ and more fully confirmed by several later studies. 159-163 In this regard, the work of Becker and Bier 159 is particularly noteworthy in thas. these authors found that a free jet from a converging nozzle was at least as effective in producing intense molecular beams as a tallored expansion from a nozzle with a diverging exit section. A sizeable body of literature pertaining to the design, quantitative characterization and application of supersonic nozzle beams presently exists. These developments are adequately described in various review articles. 153,164-167

Supersonic jets begin as a continuum flow of gas issuing from the source chamber. However, as the gas expands into a region of low pressure, the particle 
density is rapidiy reduced, resulting in a transition from continuum flow to nearly free molecular flow. Consequently, nozzle sources are akin to oven beams in that they may be used to produce a relatively collisionless unidirectional stream of sample molecules. In contrast to effusive sources, a nozzle expansion results In directed mass motion of the gas upstream of the first beam defining orifice. This aperture then serves to extract the high intensity central portion of the jet. This precollimation effect markedly increases the beam flux luto the first collimation chamber. While actual beam performances generally fall well below predictions based on the formulas of Kantrowitz and Grey 157 and Parker et. al.,$^{168}$ equivalent beam pressures of $10^{-4}$ torr are commonly achleved.

In practice, the principal causes of nozzle beam intensity attenuation are background scattering phenomena and skimmer Interactions. The extent to which each of these effects contributes to molecular beam destruction largely depends on the nozzle-skimmer. distance. At large separations, beam intensities are reduced as a result of appreclable scattering of the free fet molecules by the background gas. As the nozzleskimmer distance is shortened, background scattering diminishes in effect and intensities pise. Eeam 
performances are observed to pass through a maximum and then fall off as further reductions in the nozzle. skimmer distance are made. At still smaller separations, beam intensities typically pass through a minimum and eventually begin to rise again. This functional dependence arises from increased scattering of the jet molecules at the skimmer as well as scattering from the nozzle exhaust chamber walls. Finally, at very small nozzle-skimmer spacings, the skimmer operates in continuum flow, resulting in a large throughput and generally overwhelming pumping speed demands.

Because of their importance in limiting maximum attainable beam intensities, numerous experimental and theoretical investigations have been made with the intent of characterizing and finding ways to circumvent background scattering and skimmer interference phenomena. ${ }^{169-173}$ In general, it has been found that the deleterious effect of backeround scattering is best minimized by decreasing the expansion chamber pressure. On the other hand, skimmer interactions are somewhat more difficult to control. In this regard, considerable attention has been given to improving beam performance by optimizing the slimmer geometry. 174-177 Unfortunately, Intensity enhancements which result from changes in skimmer shape are 
typlcally falrly small. 167 For molecular beams of condensable compounds, it is possible to virtually eliminate skimmer interference by cooling the nozzle exhaust chamber walls and skimmer cone to liquid nitrogen or liquid helium temperatures; however, a practical disadvantage of this method is the frequent plugging of the beam defining orifice which necessarily occurs. In the final analysis, complete removal of the skimmer is perhaps the most desirable and definitely the most straightforward solution, but this measure requires enormous pumping speeds in the test chambers. These considerations play a cruclal role in the vacuum and hardware features of supersonic nozzle beam sources. Depending on the particular application, most current Instrumental designs represent some type of compromise between high beam intensity and the two related factors of economy anc practicality.

Iil addition to the relatively high sample Intensities which may be achieved, nozzle beams possess several other well known characteristics:

(1) Since the sample molecules uncergo collisions in a supersonic expansion, extensive energy transfer between various degrees of freedom can take place. These transfer processes lead to a conversion of the initial internal and random translational energies 
of the molecules into directed mass motion. As a result, individual molecular velocities are closely grouped about a mean flow velocity which significantly exceeds that expected for an effusive beam. $157,160-163$ (2) As the jet expands isentropically into a vacuum, the rate of molecular collisions decreases rapidly. Energy transfer becomes correspondingly less probable as the transition from continuum to nearly free molecular flow proceeds. At various points In the expansion, the probabilities of further relaxation of the vibrational, rotational and random translational degrees of freedom become prohibitively snall. Consequently, the conversion of thermal energy into directed mass motion is typically substantial but not complete. The expected variation of internal and random translational temperatures as a function of distance along the expansion (as given by Anderson ${ }^{167}$ ) is shown in Figure 6. Final temperatures are determined by the number of collisions required for the respective relaxation processes as well as by such expansion conditions as nozzle source pressure, expanston chamber pressure, etc. In general, the vibrational relaxation process is completed most quickly, followed by the rotational and then translational degrees of freedom. 


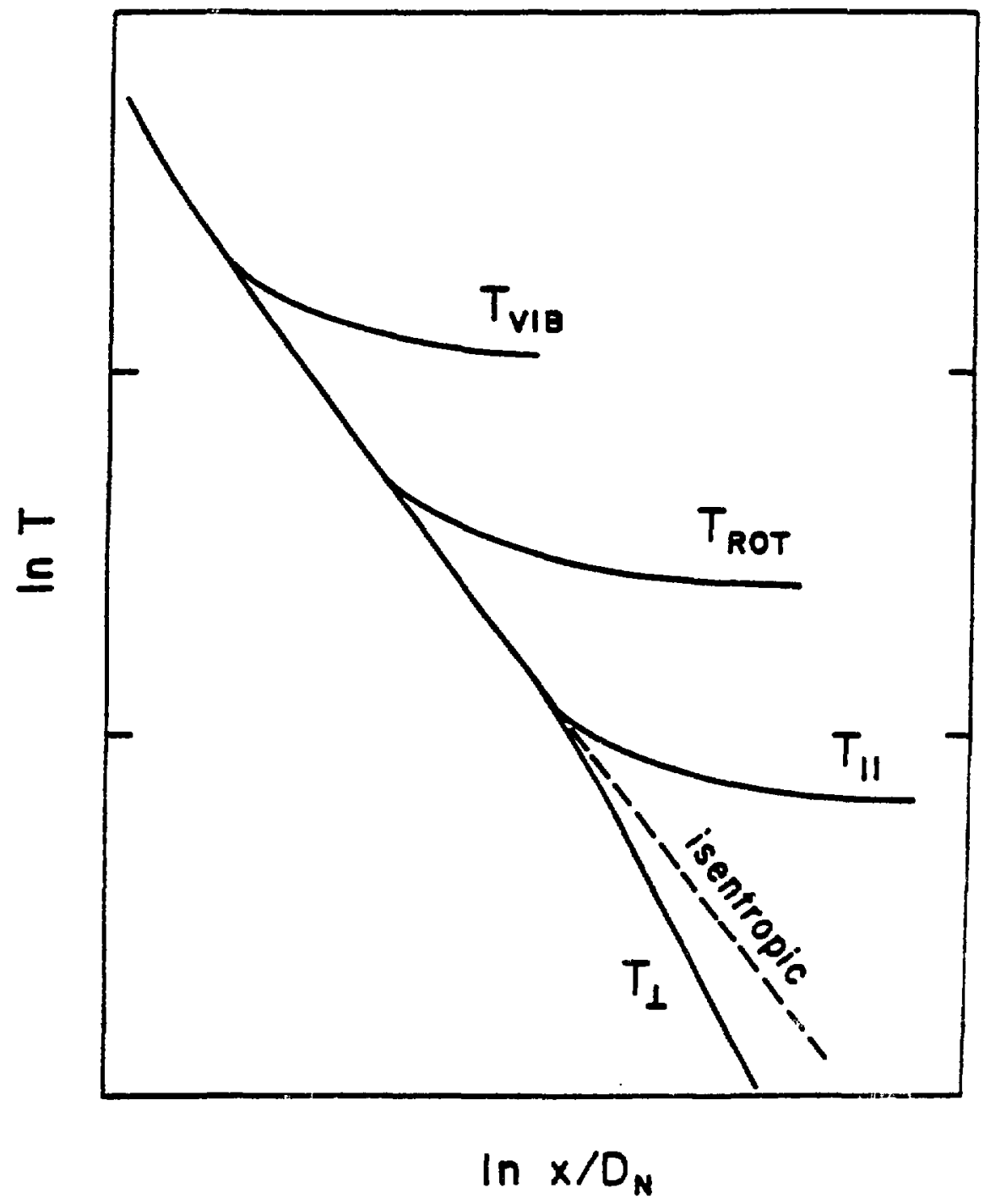

Figure 6. Varlation of Vibrational, Rotational
and Translational (Parallel and
Perpendicular to Expansion Axis)
Temperatures in a Free Jet as a
Function of Axial Distance, $x$, in
Nozzle Diameters, $D_{N}$ (from Ref. 167). 
(3) Depending, on various source conditions, supersonic expansions of gas mixtures can lead to preferential enhancement of the concentrations of one or more components along the beam axis. This mass separation erfect was apparently anticipated by DIrac 178 and was first reported by Tahourdin 179 in 1946. Under most clrcumstances, gas mixture beams are observed to be enriched in heavier specles along the centerline of the expansion. This phenomenon has come to be known. as a "positive enrichment" or "positive separation." Mass separation effects of this type arise from inter-。 actions of the gas mixture jet with the sampling.. hardware $e^{180}$ as well as from radial migration of the : heavier molecules toward the beam axis due to a pressure diffusion mechanism in the free expansion. 181-184 However, preferential enhancement of lighter components or "negative separation" can also occur. 185,186 The mechanism for this phenomenon appears to involve selective asplration of light background molecules through the shock wave structure of an underexpanded free jet. 178,187 since the background gas chlefly consists of molecules skimmed from the expansion, favorable source conditions will result in the observation of higher concentrations of the lighter species of a gas mixture downstream of the skimmer. It is clear that 
nozzle beams of mixed gases possess several advantageous features. It is obviously possible to produce intense beams of a given compound by suitably "seeding" the jet w1th another gas so that selective mass separation takes place. An important consequence of this behavior is that aerodynamic enrichment phenomena in flow regimes may be effectively utilized as a gas and isotope separation technique. 188 Finally, gas mixture jets provide a means to overcome the previously discussed energy IImitations which exist for oven beams. 153,I66 This feature arises from the fact that heavy molecules are accelerated by lighter species in a nozzle expansion such that the final velocity of a gas mixture beam corresponds to that velocity which would be expected for a unfform gas having a molecular welght equivalent to the concentration-welghted average of the various components in the mixture. Hence, the mean jet velocity of a dilute mixture of one or more heavy species in a light carrier gas will be very nearly equal to that if the pure carrier gas. By taking advantage of positive mass separation effects in tandem with this aerodynamic acceleration, falrly intense high energy beams of heavy components in mixed gases can be achleved. This method has been used to obtain supersonic nozzle expansions in the $0-37$ eV range. 189 
(4) Under appropriate expansion conditions, the low molecular temperatures produced in supersonic jets can lead to partial condensation of the sample gas. As a result, weakly bound cluster molecules can be produced in relatively large amounts. Full condensation does not occur because the rate of necessary three-body molecular collisions decreases rapidly as the expansion proceeds. At present, there exists no rigorous theory to quantitatively determine the extent of cluster formation for a given system. 190 However, the degree of observable condensation has been observed to be a function of such experimental conditions as the Inftial composition of the test gas, source pressure, source temperature, nozzle orifice diameter, expansion chamber background pressure and skimmer interaction effects. Considerable effort has been expended to arrive at empirical correlations of claster production with source parameters and various scaling laws for the kinetics of condensation in nozzle flows have been developed. 190-198 Precise correlations of this type clearly require accurate measurements of the relative concentrations of cluster species in the expansion. Unfortunately, these measurements are a non-trivial task. In particular, the mass spectrometric detection methods most often used for this purpose contain sub- 
stantial uncertainties due to the usual mass discrimination effects, as well as unknown varlations in lonization cross sections with cluster size and the largely indeterminable contributions to a given cluster ion signal from fragmentation of higher clusters. Also, It is necessary to account for any significant preexpansion equilibrium concentrations of weakly bound complexes. Nevertheless, several general trends are consistently observed in free jet studies. Definite cluster production increases often result from elevated source pressures, decreased source temperatures and larger nozzle openings. Important additional contributions from skimmer interaction effects ${ }^{199}$ are illustrated in Figure 7. Therefore, within the Iimitations of a given instrumental design, a number of parameters may be adjusted to optimize the concentrations of Individual cluster species; 1.e., dimers, trimers, tetramers, mixed clusters, etc.

Several of the features mentioned in the foregoing paragraphs promote supersonic nozzle beams as a unique and useful samping method for photolonization mass spectrometric investigations. Perhaps the most stralghtforward advantage of this technique is the facile control which can be exercised over the position and 


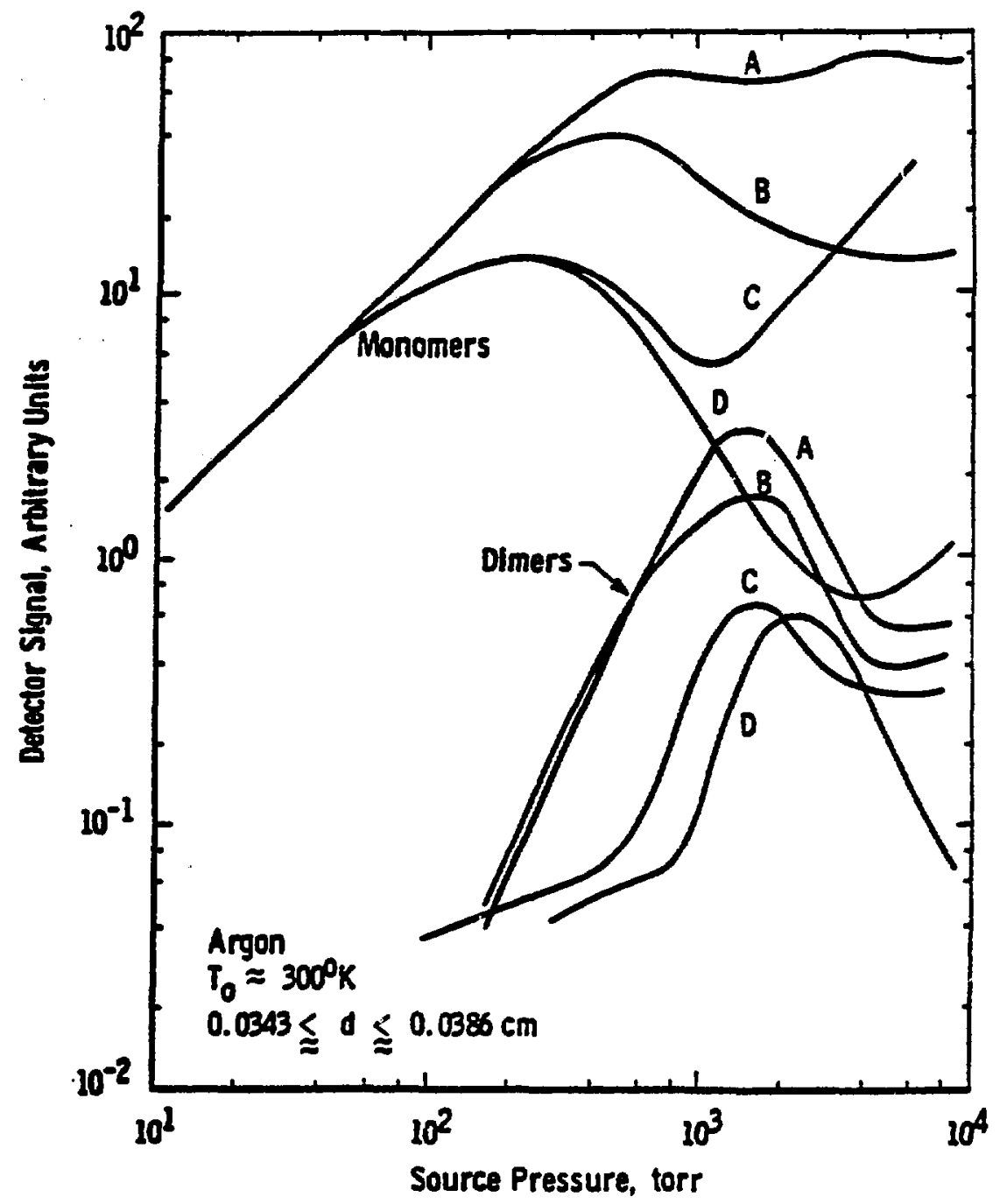

Figure 7.

Effect of Skimmer and End WaIl

Temperatures on Argon Monomer

and Dimer Beam Intensity:

$A=20 \mathrm{~K}$ Skimmer and End Wall,

$B=295 \mathrm{~K}$ SkImmer and $20 \mathrm{~K}$ End

Wall, $C=20 \mathrm{~K}$ SkImmer and

295K End Wall, $D=295 \mathrm{~K}$

SkImmer and End WaII

(from Ref. 199). 
concentration of the target gas. The former parameter is fixed by the instrumental geometry. At the same time, sample concentration is primarily determined by the varlous expansion conditions. Consequently, this factor may be held constant for hours at a time by carefully monitoring source pressure, source temperature, etc. Another fundamental advantage of molecular beams is that they provide a sampling regime which may be conveniently and alternately quenched and fully restored through the use of a beam flag. Hence, experimental background corrections are easily made. Needless to say, the relatively high intensity of supersonic jets is also a valuable asset in photolonization work, especlally in view of the fact that photon fluxes and photolonization cross sections are typically much smaller than corresponding values for electron impact methods. On the other hand, nozzle beams permst the realization of fairly large equivalent sample pressures $\left(10^{-5}-10^{-4}\right.$ torr) without unduly increasing the background pressure in the ionization region. Hence, a suitable vacuum may easily be maintained in the monochromator, test chamber and mass spectrometer. Under these conditions, it is advantageous to extract the photolons in a direction perpendicular to the molecular beam flow. 
By quickly drawing out the lons into a region of relatively low pressure, it is possible to substantially minimize the incldence of undesirable 1onmolecule reactions.

The characteristic occurrence of extensive energy transfer in supersonic expansions represents a distinct asset in photolonization studies. The principal advantage in this regard is the high effective resolution accruing to photolon yleld measurements from vibrational and rotational relaxation in free jets. This positive aspect of supersonic molecular beam sampling has been adequately discussed in previous sections. However, a related phenomenon which deserves additional consideration is the process of partial condensation. This by-product of low internal temperatures in nozzle beams is particularly useful in photolonization mass spectrometry in that it greatly facilitates the investigation of the energetics of interesting cluster molecules and their respective ions. To fully appreclate this contribution of supersonic molecular beam sampling, it is necessary to digress slightly by considering the characteristics and importance of weakly bound species.

A molecule may generally be regarded as weakly bound if it contains at least one kond which has a 
strength of $0.4 \mathrm{eV}$ or less. Most of these molecules may conveniently be classified as resulting from one of two categorles of interactions: (1) van der Waals forces and (2) the hydrogen bond. The term "van der Waals forces" is a collective designation which refers to a weakly attractive total intermolecular interaction corresponding to the complex interplay of several attractive and repulsive elements.200 A principal type of attraction in this interplay is the so-called London, or dispersion, energy which may be thought of as arising from statistically present instantaneous dipoles in atoms or molecules. The London energy is present in any collection of particles and this effect, along with exchange (Paul1) repulsion, largely accounts for the existence and equiliorium configuration of many cluster molecules. Rare gas cluster species like Ar2, $\mathrm{Kr}_{2}$ and $\mathrm{Xe}_{2}$ are essentially bound as a result of London forces alone while other attractive terms (chargetransfer stabilization, induction interactions, etc.) contribute in varying amounts to the formation of such clusters as ArClF and $\mathrm{C}_{6} \mathrm{H}_{6}-\mathrm{Cl}_{2} \cdot 200$ Hydrogen bonding, the second important class of weak intermolecular interactions, is a well known phenomenon which is cruclal to many interesting and valuable properties of water, 
ammonia and various organic compounds. This attraction princlpally occurs in molecules containing hydrogen and such electronegative constituents as nitrogen, oxygen or fluorine. A useful and falrly accurate qualitative plcture of hydrogen bonding is that $a \mathrm{H}$ atom which is covalently bound to a strongiy electronegative atom may experience a relatively small but significant electostatic attraction toward another region of high electron density. This picture is consistent in a simple fashion with the characteristically asymmetric hydrogen bond geometry $A-H---B$, where the $A-H$ covalent bond distance is much shorter than the separation between $H$ and $B$. In reality, there are at least four possible contributions (positive and negative) to hydrogen bond formation: electrostatic interaction, London-type van der Waals forces, exchange interaction and bond delocalization. 201 In any case this category of falrly weak bonding may be expected to be important in such cluster species as $\left(\mathrm{H}_{2} \mathrm{O}\right)_{2},\left(\mathrm{NH}_{3}\right)_{2}$ and $(\mathrm{HF})_{2}$.

As indicated above, one or more types of weak attractive forces are operative in any aggregation of atoms or molecules. Consequently, weakly bound molecules may be expected to be present in almost all gases, at least in trace quantities. In splte of the low concentrations in which cluster molecules are usually found 
in equilibrium systems, substantial evidence exists which suggests that these species are important in establishing many of the bulk properties of gases and are Influential in various other aspects of physical chemistry, including chemical kinetics, nucleation and condensation phenomena, energy transfer processes and spin relaxation. 200 For example, a crossed molecular beam study has convincingly demonstrated that van der Waals dimers particlpate strongly in the formation of certain interhalogens by exchange reactions.202 In this investigation, the following six-center reactions

$$
\begin{aligned}
& \left(\mathrm{Cl}_{2}\right)_{2}+\mathrm{Br}_{2}+\mathrm{Cl}_{2}+2 \mathrm{BrCl} \\
& \left(\mathrm{Cl}_{2}\right)_{2}+\mathrm{HI}+\mathrm{Cl}_{2}+\mathrm{HCl}+\mathrm{ICl}
\end{aligned}
$$

were obsepred to proceed easily at thermal collision energles (about $3 \mathrm{kcal} / \mathrm{mole}$ ) while reaction products for the straightforward four-center $\mathrm{Br}_{2}+\mathrm{Cl}_{2}$ mechanism were not observed at collision energies up to about 25 $\mathrm{kcal} / \mathrm{mole}$. Several other reactions involving weakly bound molecules are known as well.203-205 Another Interesting example is the postulated formation of small van der Wals clusters as the products of the first few addition reactions in the homogeneous nucleation of argon. 197 The following consecutive steps are suggested:

$$
\mathrm{Ar}+\mathrm{Ar}+\mathrm{Ar} \nsubseteq \mathrm{Ar}_{2}+\mathrm{Ar}
$$




$$
\begin{gathered}
\mathrm{Ar}_{2}+\mathrm{Ar}_{2}+\mathrm{Ar}+\mathrm{Ar}_{3} \\
\vdots \\
\mathrm{Ar}+\mathrm{Ar}_{\mathrm{n}}+\mathrm{Ar}_{\mathrm{n}}+1
\end{gathered}
$$

Weakly bound clusters are likely to play an equally significant role in the kinetics of other nucleation processes. 200 Because of the Importance of van der Waals and hydrogen-bonded molecules, numerous theoretical and experimental methods have been employed in order to determine tine structure and nroperties of these specles. These methods and results have been extensively reviewed. 200,206-208

Photolonization mass spectrometry in conjunction with molecular beam sampling is a powerful technique for studying weakly bound molecular and lonic complexes. The information which is provided by the measurement of cluster photolonization efficlency curves is much the same as in monomer studies. For instance, first and higher lonization potentials of cluster molecules may be obtained from the various thresholds of the appropriate photolon yleld curves. If well resolved autoionization structure is present, energy separations of exclted molecular Rydberg states may be determined. Other types of information include ionic vibrational frequencles, Franck-Condon factors for direct ionization and frazment ion appearance potentials. As indicated in 
Sections II.B.2 and II.B.3, important thermochemical data can be derived by introducing measured cluster Ionfzation and appearance potentials into appropriate thermodynamic cycles.

D. Experimental Design

The experimental techniques employed in photoionization mass spectrometric studies very much depend on the specific applications. However, a common denominator in virtually all of these applications is the need to overcome difficulties imposed by the relatively low monochromatic photon fluxes (e.g., compared to electron bombardment sources) currently obtainable. In particular, it is desirable to maximize the light throughput of the monochromator, the ion transmission and collection efficiencies of the mass spectrometer and the sensitivity of ion detection. Th1s discussion will emphasize those instrumental features which have been successfully employed in the measurement of photolonization efficiency curves. It is convenient to consider separately experimental techniques pertaining to (1) the light source-monochromator system, (2) the sampling source and ionizer, (3) the mass analyzer and (4) the ion detection system. Light Source - Monochromator The ionization potentials of the vast majorit: 
of falrly simple molecules fall in the energy range of 8-20 eV. As a consequence, vacuum ultraviolet light is required for most photolonization work. For this purpose, a wide variety of line emission and continuum light sources have been developed. 209 However, a Iiterature survey of contemporary photolonization studies would seem to indicate that there are at present three practical light. sources for the determination of photolon yleld curves. The rare gas continua excited In a capillary discharge 210 collectively represent the most widely employed type of non-discrete source. With helium as a lamp gas, useful intensities in the range 580-1100 A may be obtained. With argon, krypton and xenon, the spectral distributions cover the wavelengths 1050-1550, $1250-1800 \AA$ and $1480-2000 \AA$ respectively. Avallable intensities are generally falriy reasonable, except in the region of 950-1100 $\AA$. For most efficient results, the continua are excited in a repetitive discharge. Elther pulse-amplifying circuits or thyratrontriggered circuits may be used. With a pulsed discharge lamp, It has been estimated that about $10^{10}$ photons/secA may be obtained at 800 \& using the He Hopfield continuum.90 A second very useful source of vacuum ultraviolet light is the molecular hydrogen spectrum produced in a D. C. capillary discharge. Although the 
hydrogen spectrum below $1670 \AA$ does not qualify as a true continuum, this light source contains a series of densely packed lines which are quasi-continuous in the wavelength region 850-1670 $\AA$. This spectrum has been very carefully characterlzed by Samson, as shown in Figure 8.209 As indicated in this figure, avaliable photon intensities at typical resolutions range from about $5 \times 10^{8}$ photons $/ \mathrm{sec}$ to $2 \times 10^{10}$ photons $/ \mathrm{sec}$ at the Lyman a atomic line. Finally, synchrotron radiation is an excellent, but largely unavallable, continuous vacuum ultraviolet light source. This radiation arises from high energy electrons (or possibly other charged particles) undergoing centripetal acceleration in a magnetic fleld. At relativistic electron energies, the instantaneous distribution of emitted light is confined to a narrow cone in the plane of the particle trajectory. The axis of this cone polnts in the direction of the electron's motion. As a result, it is quite feasible to extract much of this light into a spectrograph or monochromator. In princlple, synchrotron radiation is an ideal if zht source Inasmuch as it has a smooth wavelength depencience extending from the soft $x$-ray region to the infrared. General discussions of this radiation source are available. 209,211-2i3 From photciznization experiments 


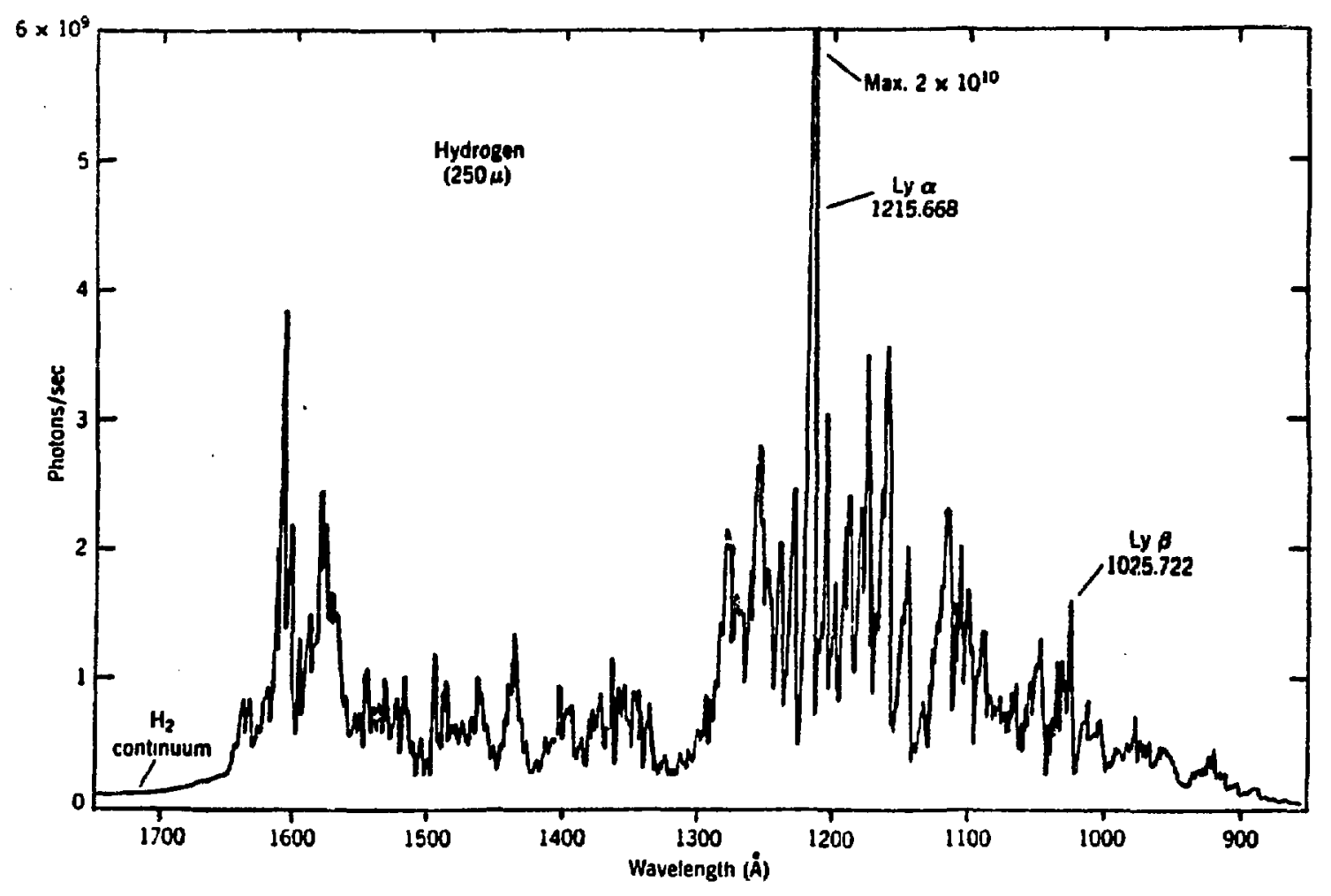

Figure 8. $\mathrm{H}_{2}$ Spectrum Between 850 and $1750 \AA$.

Lamp Pressure, 250u; D1scharge

Current, 400mA. Pt-Coated Grating,

1200 lines/mm. (from Ref. 209). 
performed at the University of Wisconsin, 79-81,91 it would appear that current synchrotron facilities are capable of providing vacuum ultraviolet photon intens1t1es that are somewhat h1gher than or at least equivalent to those obtainable with conventional discharge lamps. More ambitious electron storage ring projects, such as the one under development at Brookhaven National Laboratory, should result in even higher intensities.

A meaningful comparative analysis of the rare gas continua, the hydrogen many-Iine pseudo-continuum and synchrotron radiation necessarily entails numerous practical considerations. For instance, the advantages of the undeniably superior spectral characteristics of synchrotron radiation are substantially offset by the general unavaliablity of this photon source. In addition, careful precautions in instrumental design must be taken to avold contamination of the high vacuum (about $10^{-10}$ torr) required for this light source. 212 Because high photon intensities are produced over a very large energy range, undesired contributions from hizher order reflections from the grating must also be taken into account. In contrast, the many-Iine spectrum obtained from a molecular hydrogen D.C. capillary discharge is in prini:ne ciearly inferior for high 
resolution photolon yleld curve applications. As mentioned in Section II.3.2., the possible experimental artifacts arising from the use of a source containing semi-discrete lines must be consldered. Furthermore, photolonization studies must be restricted to the wavelength region of $900-1650 \AA$. However, the hydrogen spectrum offers the advantage of being by far the most conveniently obtained. Caplilary discharge lamps and appropriate regulated D.C. power supplies are both commercially available and falrly simple to operate. Since no suftable materials exist for light transmission below $1040 \AA$, it is best to be able to operate a monochromator without windows. Inasmuch as the $\mathrm{H}_{2}$ quasi-continuum can be intensely excited at lamp pressures as low as a few hundred microns, a hydrogen source may be easily coupled into a system of this type. In other words, a good vacuum may be maintained in the monochromator with a relatively modest differential pumping design. Moreover, a properiy operated lamp will work for many hours with very little souttering. The spectral quality associated with rare gas continua lies in between the smooth continuum of synchrotron radiation and the many-line hydrogen emission. In a similar manner, the experimental techniques for producing repetitive rape gas aischarges are more accessible 
than electron storage ring facilities, but somewhat more difficult than $\mathrm{H}_{2}$ D.C. discharge methods. Although they are falrly expensive, the necessary power supplies and pulsing equipment are commercially avallable. More Important, high lamp pressures (about 40-100 torr) are required for intensely exclted continua. For krypton and xenon, sealed lamps with windows are typically employed. A hellum lamp, on the other hand, must be operated windowless. Consequently, the monochromator differential pumping requirements are quite severe. The use of high purfty gases is advisable in these discharges as is the Incorporation of a back-pumping port at the lamp cathode. This latter procedure prevents the narrow monochromator entrance slit from being clogged by significant amounts of sputtering deposits.

From a practical instrumental standpoint, it Is clearly advantageous to perform photolonization studies with a vacuum ultraviolet monochromator in which selected and varlable light dispersion is accomplished without movement of the entrance and exit slit assemblies. Two commonly employed monochromator classes are the Seyz-Namioka type, 214-216 in which the wavelength scan is jrovided by simple rotation of a concave diffraction grating about a vertical axis throlith the grating enter and the near-normal Inci- 
dence type, ${ }^{217}$ in which the wavelength is varied by rotation and translation of the grating along the bisector of the angle subtended by the slits at the center of the grating. With regard to photoion yield curve experiments, the principal asset of the SeyaNamioka mounting is the wide angle (about $70^{\circ}$ ) between the entrance and exit arms. In comparison with the near-normal incidence type, this configuration permits considerably more freedom in apparatus design. Unfortunately, conventional Seya-Namioka monochromators are by no means equal to near-normal incidence mountings in terms of ultimate resolution and maximum dispersed light throughput. Iosses in these categories ailse primarily because of characteristically large astigmat1sm effects. Substantial improvement in the performance of the Seya-Namioka design can ke achieved through the use of curved silts. 218

For a given monochromator, the physical

properties of the grating which most affect the throughput of the instrument are the blaze angle, the surface coating and the density of grooves. The first property is determined in the manufacturing process 209 and does not require further discussion here. W1th regard to surface fllms, an approprlate coating should be chosen for the rezion of interest since the refler- 
tances of the most commonly used materials vary considerably over the vacuum ultraviolet wavelength range. 126 For work in the region of 1150-1500 , an aluminum-coated grating with a thin $\mathrm{MgF}_{2}$ overcoating appears to be the best choice. These materials provide reflectances as high as $80 \%$ between $1200-1500 \AA$. The reflectance properties of LiF are quite good (55-60\%) in the range of 1000-1200 $\AA$, but an overcoating of this substance is hygroscopic and must be protected from moisture. At wavelengths shorter than $900 \AA$, the use of gratings containing surface films of gold or platinum is advisable. At the same time, the groove density of a grating affects the throughput of a monochromator in that the resolution obtained with given entrance and exit slit widths is directly proportional to the number of rulings. That is, a grating with twice as many grooves permits approximately the same resolution at twice the slit size. On the other hand, if the lamp spectrum is constant as a function of wavelength, the intensity of the dispersed light leaving the exit arm is proportional to the square of the slit widths. Consequently, Increasing the groove density by a factor of two can result in as much as a fourfold enhancement in monochromatic photon intensity. It should be noted that practical limitaticns on the spacings in classically 
ruled diffraction gratings exist because of the tendency for ghosts and stray light levels to increase with groove density. This is apparently not the case for holographlcally recorded gratings ${ }^{126}$ and the production of very finely spaced optical components is possible through this technique.

Dispersed light from the grating is focused at the monochromator exit s.lit and then diverges. In order to effectively apply this monochromatic radiation to photolonization, it is necessary either to place the exit arm of the instrument as near the ionization region as possible or to refocus the diverging beam into the fonfzer by means of an appropriate mirror. Each of these methods has certain disadvantages. For examele, special insulating precautions to prevent arcing must be taken if the former technique is used in conjunction with an ion box maintained at high voltage. Also, physically incorporating the monochromator into the mass spectrometer test chamber makes a variable slit assembly somewhat inaccessible. From the standpolnt of practical design, it is much easier to position the monochromator outside the ionization region. Now, the use of a refocusing mirror clearly introduces an additional reflecting surface and therefore intensity losses. However, these losses can be minimfzed ir utilizing a grazing 
incidence optical configuration. This technique has been employed in the present study (see Section III.B.). The final aspect of a monochromatic photon production system is the process of light detection. This measurement is generally accomplished by using one of two established methods. In the first monitoring technique, the transmitted light is allowed to impinge on a window covered with a thin layer of fluorescent material. A photomultiplier is then used to determine the fluorescent intensity. Because of its relatively constant quantum efficiency at wavelengths below $1300 \AA$, the most commonly employed phosphor is sodium salicylate. Several other substances may also be used, including diphenylstilbene, tetraphenyl butadiene and terphenyl. An excellent discussion of the characteristics of various fluorescent materials is found in Samson. 209 The second monitoring method consists of a simple photoelectric detector having a nickel photocathode. The success of this technique rests on the fact that the photoelectric yield of a heat treated nickel cathode is nearly constant in the wavelength range of $473 \AA$ to $1000 \AA .219$

\section{Sampling System and Ionizer}

Sample introduction in ordinary mass spectrometry usually involues some pye of gas-handing 
system and a leak arrangement to the fonfzer. For photolonization efficiency curve measurements, it is particularly important to maintain a constant source pressure over substantial lengths of time. The need to minimize ion-molecule reactions and collisional removal of fons from the mass analyzer typically places an upper limit on the practical sample pressures which can be employed. Detalls of conventional sampling techniques may be found in any of numerous books on mass spectrometry. 220,221 The many advantages of supersonic molecular beam sampling methods in photoionlation mass spectrometry have been examined previously. Varlations in nozzle beam production systems are generally fairly minor and the sampling system described in Section III.A. closely follows standard instrumental design features for this technique.

The most distinctive structural characteristics of an ion source used in photoionization are the entrance and exit holes which provide for necessary passage of the monochromatic light through the ionization region to the photon monitor. With molecular beam sampling, a similar passageway (along the beam axis) must be included to avoid concentration bulld-up of the target gas. Otherwise, there is considerable instrumental and electrical desian freejom die to the many satisfing asoects o: a 
photon beam as an fonizing medium. In contrast to electron impact methods, large ion draw-out fields can be utilized without affecting the photon energy. Space charge effects are confined to the very small amount arising from the photolonization processes. Furthermore, the ablity to perform 1onization without the use of a hot fllament allows the fon box to be maintained at any reasonable desired temperature during the whole course of an experiment. For efficient ion collection, it is advisable to cross the light beam with the sample gas as near the ion exit port as possible. Also, 1 is desirable to include at least a crude electron gun to facilitate inftial tuning of the mass spectrometer, especially for those minor constituents giving rise to small photolon signals. FinalIy, it is important to guard against lonization from high energy photoelectrons originating outside the fon source. As mentioned previously, this problem can usually be eliminated by an approprlate set of collectors.

\section{Mass Analyzer}

In view of the low photolon signals tyoically encountered in photolonization efficiency curve determinations, a cruclal feature of any mass spectrometer employed for this purpose is hi in ion transmission. 
On the other hand, mass resolution requirements are generally falrly modest (e.g., about I amu in 100-400). Two mass analyzer types which have found wide application are quadrupole mass spectrometers and magnetic deflection instruments. The former type of analyzer has developed from the inftial demonstration that a quadrupole electric field can act as a variable bandpass mass filter. 222 Design criteria and equations of Ion inotion in this type of fleld are well characterlzed. 223 Excellent medium resolution quadrupole mass spectrometers are commercially avallable. These instruments are fairly compact and generally have very good transmission properties, Including a wide acceptance angle for ion trajectories. 224 Adequate ion transmission can also be achieved with otherwise conventional magnetic spectrometers equipped with special ion optical features. $54,225,226$ A particularly useful and elegantiy simple device in this regard is the electrostatic quadrupole lens system designed by Giese.227,228 Ion transmission efficiencies approachIng unity have been clalmed 55,140 for a normal-entry homogeneous magnetic-sector instrument using these focusing lenses.

Ion Detection.

In eary ghotoionization oxperiments, ion 
detection was usually accomplished by directly measuring the anode current of a multi-stage electron multiplier (e.g., with a vibrating reed electrometer). While this technique is perfectly adequate for conventional photolonization studies of major constituents in a sample gas, more sensitive detection methods are required for photoion yleld curve measurements of species which are present in low concentration. The development of pulse counting techniques represents a vitally important instrumental advance which has markedly increased the sensitivity of ion detection. In one technique, fons are allowed to impact the electron multiplier directly. Voltage pulses produced at the anode of the multiplier are typlcally amplifled, shaped and analyzed for height. The pulse height analysis (discriminator) steo serves to selectively pass signal pulses, eliminating many of the weaker dark current pulses which have passed through fewer multiplier stages. Finally, those pulses which are accepted in the discriminator stage are counted. Random fluctuations in the data may be reduced by employing longer counting times. The use of low photon energies in conjunction with the low monochromatic photon intensities generally obtainable frequently results in extremely small backround count rates. Consequently, under ârorabie conditions, signals as low az 
0.03-0.1 count/sec can be detected and reoroduced. A second very sensitive counting method involves the use of the Daiy scintillation detector. 229 Ions entering this detector are accelerated through some $30-40 \mathrm{keV}$ and Impinge on an aluminum-coated target which ejects several secondary electrons per lon. The number of electrons produced at each impact depencis on the mass of the incoming lons. The secondary electrons are then accelerated by the same electric fleld and strike an organic plastic scintillator covered with a very thin vacuum-deposited reflecting and conducting layer of aluminum. Electrons pass through the Al film to the plastic phosphor with negligible energy loss. The presence of the film allows the face of the scintillator to be grounded and provides for increased light collection efficiency. The light generated by the phosphor is detected by a photomultiolier which may be convenlently positioned outside the vacuum system. Voltage pulses are then analyzed and counted as before. 


\section{APPARATUS}

The apparatus used in this study consists of a moderate resolution mass spectrometer which has been modiffed to include a supersonic molecular beam sampling system and a photon Ionfzation source. The varlous instrumental design elements will be consldered first, followed by a description of the data collection process in a typical experiment.

A. Nozzle Beam Source and Iontzer

- The nozzle beam system and ionization region are shown in Figure 9. A supersonic molecular beam is obtalned by expanding a gas through a 0.004 " diameter nozzle orfifice at stagnation pressures of up to 2000 torr. The nozzle exhaust chamber is pumped by two 4" Stokes Model 150-I ring jet booster pumps which maintain a vacuum of about $10^{-3}$ torr. Forepumping is accomplished by two Welch $1397 \mathrm{~B}$ mechanlcal pumps. The nozzle was machined from nickel and mounted in a boron nitride support. The nozzle is also equipped with a bevel gear arrangement to permit translation of the orffice along the beam axis. This translation can be controlled from outside the vacuum system. The boron nitride is laced with small heating wires so that the assembiy can be operated above room temperature. The nozzle sas inlet 


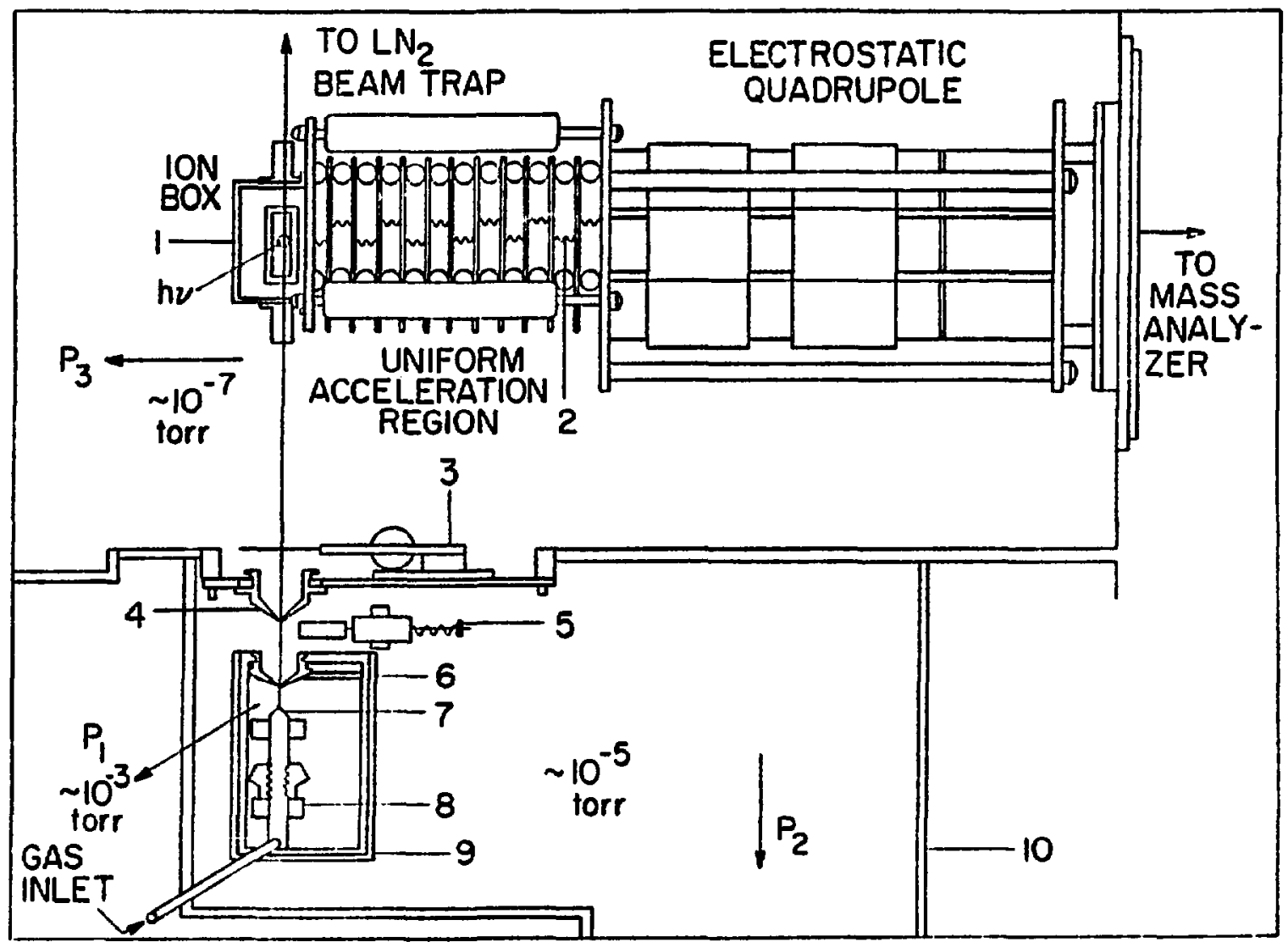

Ëure 9. Nozale Eeam Sampling and Ion Source: (1) Fon Box at +10 ht (see text), (2) $12 x$ is vis to Erovide uniform Accezeration of Ions over $10 \mathrm{k}:$, (3) Tuning, Eork Chopper, (4) Secondary Skimmer, (5) Electromagnetic Beam Flag, (5) Enimary Skimmen and Skimmer support, (7) Nozzle, (3) Nozzie Support and Bevel Gears for Nozzie Advance, (9) Wozzle Exhaust Chamben, (10) Second Differential Pumping Chamber: ( $\left.P_{I}\right) 575$ I1ters/sec (see text), $\left(P_{2}\right) 700$ Iiters.'sec (see text), ( $\left.P_{g}\right)$ I'to0 liiers) sec 6" Diffusion Pump. 
tube is connected to the external gas handing arrangement by flexible tubing coupled to a feedthrough in the expansion chamber wall. A simple three-way gas manifold allows the introduction of more than one compound if desired. For pure gases or gas mixtures, sample pressure is governed by metering valves in addition to the twostage regulators on the gas bottles. A fixed vapor pressure of liquid samples is maintalned by carefully controlling the source temperature. Stagnation pressures are monitored by a Texas Instruments precision pressure gauge.

The nigh intensity central portion of the free jet expansion is extracted with a $0.018^{\prime \prime}$ diameter conical skimmer into a second differential pumping region. This chamber is pumped by a 4" NRC Model ViS-4 diffusion pump to a pressure of less than $10^{-5}$ torr. The skimmer was machined from stainless steel to a length of $7 / 16^{\prime \prime}$ with external and internal semi-apex angles of $40^{\circ}$ and $30^{\circ}$, respectively. The expansion is further collimated by a $0.040^{\prime \prime}$ conical skimmer before entering the main chamber. An electromagnetic plunger, situated between the primary and secondary skimmers, serves an an effective beam flag. Also fabricated from stainless steel, the secondary collimator is 5/3" long and contains external and interior angles of $50^{\circ}$ and $45^{\circ}$, respectively. Downstream of this 
skimmer, the molecular beam oasses through a small box where lons may be produced elther by electron bombardment or by photon absorption. A Ifquid nitrogen-cooled surface is used to trap those molecules which escape Ionization. A Ifquid nitrogen-trapped $6 "$ CVC Model PMC-6B diffusion pump maintains the main chamber pressure at less than $3 \times 10^{-7}$ torr when the beam is running. A Welch $1397 \mathrm{~B}$ mechanlcal pump $(17.7 \mathrm{cfm})$ is used to back the diffusion pumps in both the Ionization chamber and the second differential pumping region.

The small ion box is gold-plated and contains entrance and exit ports for dispersed ultraviolet light, a tungsten fllament arrangement to provide electron bombardment capability, and an inner surface to which a small Ion repellin, voltage $(+0-20 \mathrm{~V})$ may be applied. Electron bombarament is controlled by a Model ER-1 emission regulator (IASL PN\$200426). In addition to regulating the emission current of $10-200 \mathrm{eV}$ electrons, this unit provides biasing voltages to the electron collector, fllament shield and ion repeller and also monitors the trap (collector) current. A bar magnet (not shown in Figure 9) is positioned directly in back of the Ion box to focus the electrons into the path of the molecular beam. When photolonization is used, the light beam and rolecuish beam oross at right anoles. In this 
mode, the emission regulator operates on a dummy load (a 5Y3GT full-wave vacuum rectifler) and mainly serves as a source of repeller voltage. In elther case, the ion box and emission regulator are designed to "float" at $+10 \mathrm{kV}$. Positive 1ons extracted from the box are uniformly accelerated (see Figure 9) by the $10 \mathrm{kV}$ potent1al into a pair of quadrupole lenses 227,228 which focus the ions Into a Iine Image at the mass spectrometer entrance slit. Lens parameters have been calculated from the formulas of Lu and Carr. ${ }^{228}$ variable voltages in the appropriate ranges (about $\pm 283 \mathrm{~V}$ and $\pm 204 \mathrm{~V}$ ) are provided by $a$ quadrupole lens blas power suppiy (LASL PN\#205346). In order to maximize the beam number density in the ionization region, it is necessary to align the nozzle orifice, the primary and secondary skimmers and the Ion box molecular beam entrance and exit slits such that all of the openings lie along the same axis. It proved to be convenient to use a portable He-Ne laser for this purpose. As a first step, the laser was mounted on a flange directly over the ion source. A level was used to approximately orient the laser beam along a vertical axis passing through the center of the ion box exit slit. In this configuration, the laser beam was observed to pass very nearly through the center of the narrower entrance silt also. It was possibie to obtair a suffictently good 
alignment by slightly widening the entrance slit with a f1le. Now, the chamber separator containing the secondary skimmer was designed with fairly wide slots In place of clearance holes for the mounting screws. Consequently, it was possible to significantly adjust the position of the skimmer hole. Th1s adjustment capablity enabled the laser beam to be directed through the collimating orifice. Fine adjustment was made by visually maximizing the observed laser beam reflection. At the same time, the nozzle, boron nitride block and primary skimmer were designed to be mounted together in a stainless steel support. A satisfactory alignment of the nozzle and skimmer orifices was confirmed by using another He-Ne laser. The assembly contalning the nozzle and skimmer was placed in the apparatus as aligned above and positioned such that a laser beam reflection could be seen on the nozzle tip. That 1s, it was possible to shine a laser through all five relevant openings. Final adjustment was made in accordance with the visual maximum of the spot on the nozzle tip.

B. Monochromatic Vacuum Jitraviolet Source The sallent experimental design features of the light source-monochromator system used in this study are illustrated in Figure 10. The many-line spectrum of $\mathrm{H}_{2}$ 


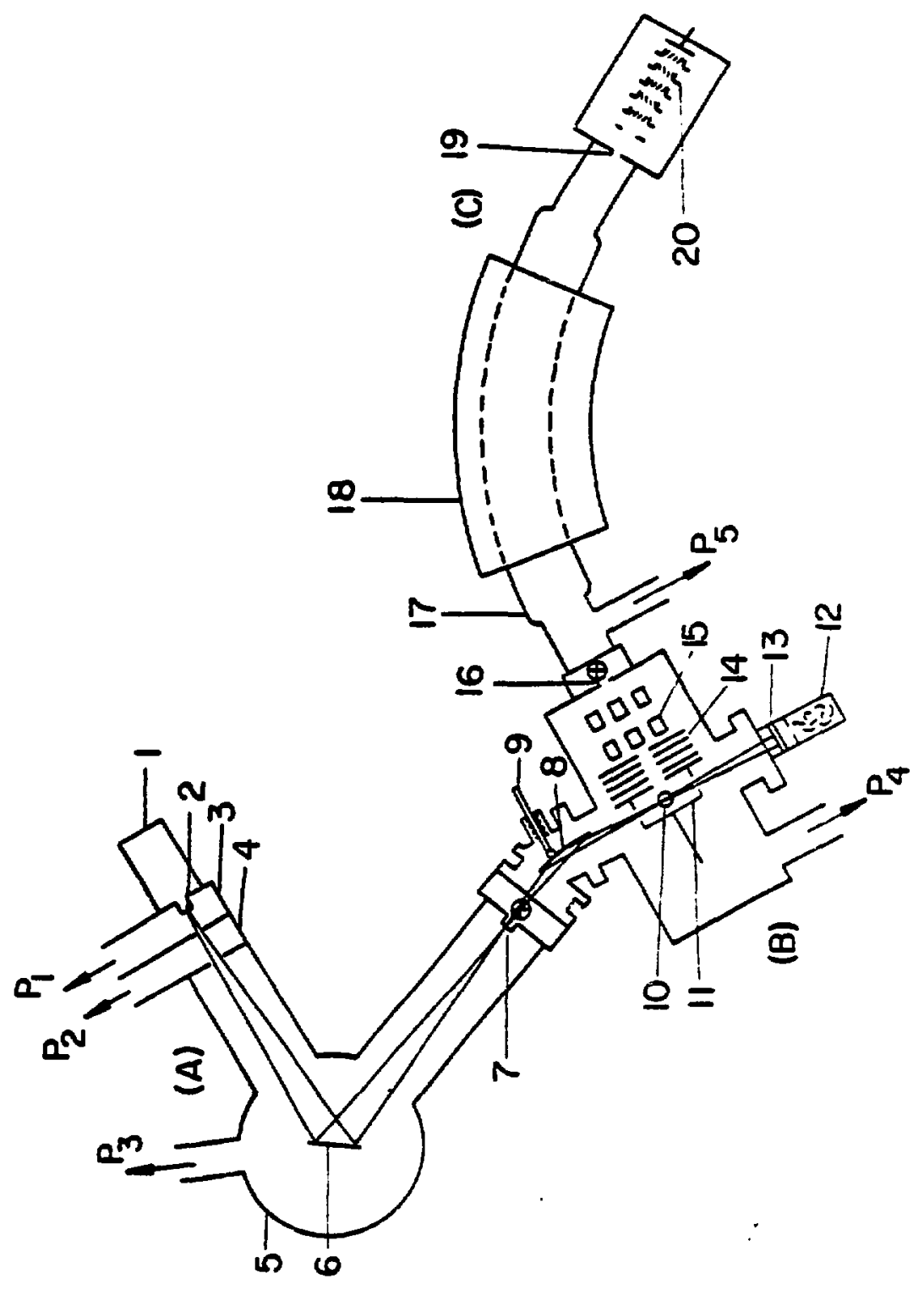


F1gure 10. Schematic Diagram of Photolonization Mass Spectrometer: (A) Light Source-Monochromator System, (B) Ionization Chamber, (C) Mass Analyzer and Detector; (I) McPherson Model 630 D.C. Capillary Discharge Lamp, (2) Monochromator Entrance SI1t, (3) First Differential Pumping Chamber, (4) Second Different1al Pumping Chamber, (5) 1/2-Meter Seya-Namioka Vacuum Ultraviolet Monochromator, (6) Grating, (7) Monochromator Exit Slit, (8) Grazing Incidence Alignment Mirror, (9) Micrometer for Mirror Adjustment, (10) Molecular Beam Crosses Photon Beam at Right Angles, (11) Ion Box, (12) Photomult1plier Tube, (13) Sodfum Salfcylate coated Window, (14) Uniform Acceleration Region, (15) Electrostatic Quadrupole, (16) Mass Spectrometer Entrance SI1t, (17) Drift Tube, (18) $60^{\circ}$-Sector Electromagnet, (19) Mass Spectrometer ExIt Sl1t, (20) Electron Multiplier; $\left(P_{I}\right) 47$ Ifters/sec Roots Blower, $\left(P_{2}\right) 700$ liters/sec 4" Diffusion Pump, $\left(P_{3}\right) 700$ ilters/sec 4" Diffusion Pump, $\left(P_{4}\right) 1400$ liters/sec 6" Diffusion Pump, $\left(P_{5}\right) 700$ liters/sec 4" Iffrusion Pump. 
produced by a McPherson Model 630 D.C. capillary. discharge lamp is used as a photon source. In view of the performance results of Cann, 230 the standard $6-\mathrm{mm}$ discharge cap1llary is replaced by one with a 3-mm bore. Exc1tation is provided by a McPherson Model 730 D.C. power supply. In order to extend the obtainable photon wavelength range below $1040 \AA$ (the transmission limit for lithlum fluoride), the light source-monochromator system is required to operate without windows. Consequently, the entrance arm of a Jarrell-Ash Model 78-650 1/2-meter Seya-Namioka vacuum ultraviolet monochromator has been modified to include two stages of differential pumping. These stages consist of chambers which are separated by slits just wide enough to pass all of the available light. In addition, the lamp is equipped with a plunger which is designed to seal against the entrance slit jaws of McPherson monochromators, limiting the $\mathrm{H}_{2}$ gas leak to an area of the slit width $\times 5 \mathrm{~mm}$. It was necessary to fabricate a special piunger in order to properly mate the McPherson lamp with the Jarrell-Ash instrument. The first differential pumoing region (monochromator entrance slit cavity) is evacuated by a small Leybold-Heraus R-152 Roots blower backed by a Welch $1397 \mathrm{~B}$ mechanical pump. The pump-out lead is wrapped with cooling coils and locsely packed with copper 
strips to inhibit warm oll vapor from rising into the monochromator. The entrance slit cavity communicates with the second differential pumping region through a $2-\mathrm{mm}$ wide opening. The vacuum in the second cavity is maintained by a 4" CVC Model PMC-4B diffusion pump equipped with a water-cooled baffle. Forepumping is again provided by a Welch $1397 \mathrm{~B}$ pump. Finally, a 6-7mm wide slit passes the light into the main monochromator chamber. This region is pumped by a liquid nitrogentrapped 4" CVC Modei PMC-4B diffusion pump. The mechanical pump employed for the lonization chamber (see Section III.A.) is used for this region also. Thus, $\mathrm{H}_{2}$ is allowed to flow through the lamp and is pumped out through the entrance slit by the system described above. With the lamp operated at $\mathrm{I}$ torr $\mathrm{H}_{2}$ and with the entrance slit at $200 \mu$, the normal operating pressure in the monochromator is $2.5 \times 10^{-5}$ torr.

The actual performance of the monochromator differential pumping system described above is very close to that predicted by simple pumping speed calculations. The necessary equations for these calculations may be found in Samson. 209 From the kinetic theory of gases, the molecular mean free path I is given by

$$
I=\frac{1}{n \pi d^{2} \sqrt{2}}
$$


where $d$ is the molecular diameter in centimeters and $n$ is the number of molecules per $\mathrm{cm}^{3}$. For hydrogen, $d$ is about $2.4 \times 10^{-3} \mathrm{~cm}$. A useful expression for $\mathrm{n}$ is $(\mathrm{P} \mu / \mathrm{T}) \cdot\left(9.7 \times 10^{15}\right)$ where $\mathrm{P} \mu$ is the pressure in microns and $T$ is the temperature in $K$. Therefore, the mean free path of $\mathrm{H}_{2}$ upstream of the monochromator entrance slit ( I torr lamp pressure) is approximately

$$
\begin{aligned}
L & =\left\{\sqrt{2} \cdot \pi\left(\frac{1000 \mu}{300 \mathrm{~K}}\right)\left(9.7 \times 10^{15}\right)\left(5.76 \times 10^{-16} \mathrm{~cm}^{2}\right)\right\}^{-1} \\
& =0.012 \mathrm{~cm} \\
& =120 \mu .
\end{aligned}
$$

That is, $L$ is very nearly as large as a typlcal entrance slit width, w. Moreover, in each differential pumping region, I may be expected to be larger than the width of the appropriate slits. Now, for $L>w$, the gas flow may be considered to be free molecular. Consequently, the pressure in each cavity of the vaculum system may be roughly estimated using the following molecular flow expression for the conductance, $C$ :

$$
C=3.6 \mathrm{~A}_{\mathrm{s}} \sqrt{\mathrm{T} / \mathrm{M}} \text { I1ters/second }
$$

where $A_{S}$ is the slit area in $\mathrm{cm}^{2}$ and $M$ is the molecuiar welght. If a $200 \mu$ entrance sitt is used, the conductance $c_{1}$ of the first slit is

$$
\begin{aligned}
c_{1} & =3.6(0.02 \times 0.5) \sqrt{30072.02} \\
& =0.439 \text { liters/second. }
\end{aligned}
$$


The area of the second slit is $0.2 \mathrm{~cm}$ (width) $\times 1.27 \mathrm{~cm}$ (height) $=0.254 \mathrm{~cm}^{2}$. The conductance $c_{2}$ is

$$
\begin{aligned}
c_{2} & =3.6(0.254) \sqrt{300 / 2.02} \\
& =11.14 \text { 11ters/second. }
\end{aligned}
$$

Finally, the area of the third slit is $0.7 \mathrm{~cm}$ (w1dth) $\mathrm{x}$ $1.27 \mathrm{~cm}$ (helght) $=0.889 \mathrm{~cm}^{2}$. The corresponding conductance $\mathrm{C}_{3}$ is then

$$
\begin{aligned}
c_{3} & =3.6(0.889) \sqrt{300 / 2.02} \\
& =39 \text { I1ters/second. }
\end{aligned}
$$

The pressure in the first differential chamber, $P_{1}$, is given by

$$
P_{1}=\frac{P_{0} C_{1}}{C_{1}+S_{1}}
$$

where $P_{0}$ is the lamp pressure and $S_{1}$ is the effective pumping speed in the chamber. The remalning pressures may also be calculated from analogous equations. Since each vacuum pump is equipped with some type of cooled baffle, the effective pumplng speeds $S_{1}, S_{2}$ and $S_{3}$ are significantly lower than the nominal maximum speeds. Reasonable estimates for these quantitles are $35 \mathrm{l} / \mathrm{sec}$, $350 \mathrm{I} / \mathrm{sec}$ and $350 \mathrm{I} / \mathrm{sec}$ respectively. Fence, at $I$ torr $\mathrm{H}_{2}$ in the lamp,

$$
\begin{aligned}
P_{1} & =(1 \text { torr }) \frac{0.439}{0.439+35} \\
& =1.2 \times 10^{-2} \text { torr. }
\end{aligned}
$$


Further,

$$
\begin{aligned}
P_{2} & =\left(1.2 \times 10^{-2} \text { torr }\right) \frac{11.14}{11.14+350} \\
& =3.7 \times 10^{-4} \text { torr. }
\end{aligned}
$$

Lastly, the pressure in the main chamber of the monochromator should be

$$
\begin{aligned}
P_{3} & =\left(3.7 \times 10^{-4} \text { torr }\right) \frac{39}{39+350} \\
& =3.7 \times 10^{-5} \text { torr. }
\end{aligned}
$$

Given the various assumptions, this calculated value is in excellent agreement with the monochromator operating pressures observed in practice.

The principal optical component is a $\mathrm{MgF}_{2}$ coated, 2400 IInes/mm Jobin-Yvon holographic grating blazed at $1200 \AA$. A large groove density is employed In order to increase the throughput of dispersed light as mentioned in Section II.D. For the most part, grating a.lignment was performed in accordance with the JarreliAsh monochromator instruction manual. Light dispersed from the grating is focused on the monochromator exit slit and refocused into the center of the lonizer by a platinum-coated $80^{\circ}$ angle of incidence alignment mirror. A metal plate containing an aperture for transmitting the light is placed between the alignment mirror and Ionizer and floated at $+30-50 \mathrm{~V}$ in order to collect photoelectrons arising from the mirror surface. The mirror desizn is based on calculations by Berlyn Brizner 
of Group M-5 at LASL. This optical component has a radius of curvature of about $1000 \mathrm{~mm}$ and is about $110 \mathrm{~mm}$ long, $35 \mathrm{~mm}$ high and $10 \mathrm{~mm}$ wide. An adjustable mirror mounting was fabricated so that a sultable focus could be achleved in the fonizer. Final alignment was accomplished by establishing the molecular beam axis with a He-Ne laser in the manner described in Section III.A. and adjusting the focusing mirror until a sharp line Image could be superimposed on the leser beam as observed on a plece of frosted glass. The astigmatism of this optical configuration is severe enough that some of the dispersed light impinges on the outside of the ion box. However, it is estimated that at least $60-80 \%$ of the photon beam is directed into the ion source. After passing through the ionizer, the dispersed light strikes a sodium salicylate coated window. Because of the low density (about $10^{-4}$ torr) of the sample and the characteristically small cross sections for photon absorption, the photon beam is essentially unattenuated by the molecular beam. The transmitted photon Intensity is determined by using an RCA 6199 photomultiplier to monitor the resulting sodium salicylate fluorescence. The quantum efflclency of a fresh coating of $\mathrm{NaC}_{7} \mathrm{H}_{5} \mathrm{O}_{3}$ is known to exhibit the following 
consistent behavior: a constant yleld from 400-1250 and a gradual monotonic increase of about $20 \%$ from $1250 \AA$ to $1500 \AA .23 I$ A picoammeter is used to measure the current output from the photomultiplier. Signals are typically one to two orders of magnitude higher than the photomultiplier dark current.

The lamp output is observed to vary signiflcantly with $\mathrm{H}_{2}$ pressure. At very low pressures $(<300 \mu)$, the discharge appears quite pale and measured Intensitles are unformly low. Upon raising the pressure from $300 \mu$ to about $700-1200 \mu$, a bright pink discharge is obtained and substantial increases in the molecular hydrogen peak intensities result. Above 1.2 torr, the discharge turns much more red and the atomic IInes (Iyman $\alpha$, Iyman $\beta$, etc.) are seen to be enharced while the molecular hydrogen spectrum diminishes in intensity. In the $\mathrm{H}_{2}$ pressure range of 7-10 torr, the lamp changes to bright pink once again with an attendant gain in the molecular hydrogen lines being observed. Further pressure increases produce strlations in the discharge capillary and Instability occurs. The most satisfactory operating regime in terms of the $\mathrm{H}_{2}$ load on the rest of the vacuum system as well as overall

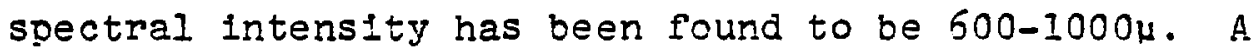


part of the $\mathrm{H}_{2}$ spectrum obtainable with the present apparatus is shown in Figure 11. With $200 \mu$ entrance and exit slits, the resolution is approximately $1.5 \AA$ or $12 \mathrm{meV}$ at $1200 \AA$. A comparison of this spectrum with Samson's results 209 in Figure 8 shows an excellent peak-to-peak correspondence in the 1160-1280 \& range. An estimate of the absolute photon intensity at a given wavelength may be calculated from the response characteristics of the type of photomultiplier used and from measured values of the absolute quantum efflciency of sodium salicylate. With $200 \mu$ slits, the current output from the photomultiplier at Lyman $\alpha$ is approximately $10^{-5}$ ampere at $600 \mathrm{~V}$. The typical sensitivity of RCA 6199 photomultipliers at $600 \mathrm{~V}$ is 0.7 ampere/lumen for 4400 \& light. ${ }^{232}$ This quantity is sultable for the above photomultiplier signal since $4400 \AA$ corresponds closely to the fluorescent emission maximum of $\mathrm{NaC}_{7} \mathrm{H}_{5} \mathrm{O}_{3}$ at $4200 \AA .209$ Now,

$$
(0.7 \text { ampere } / \text { lumen }) \cdot(804 \text { Iumen } / \text { watt })=563 \frac{\text { amperes }}{\text { watt }} \text {. }
$$

Therefore,

$$
\left(10^{-5} \text { ampere }\right) \cdot\left(\frac{1}{563} \frac{\text { watt }}{\text { ampere }}\right)=1.78 \times 10^{-8} \text { watt. }
$$

For $4400 \&$ photons,

$$
\begin{aligned}
E & =h v=\left(6.626 \times 10^{-34} \mathrm{joule} \cdot \mathrm{sec}\right) \cdot\left(\frac{3 \times 10^{10} \mathrm{~cm} / \mathrm{sec}}{4.4 \times 10^{-5} \mathrm{~cm}}\right) \\
& =4.52 \times 10^{-19} \text { joule/photon. }
\end{aligned}
$$




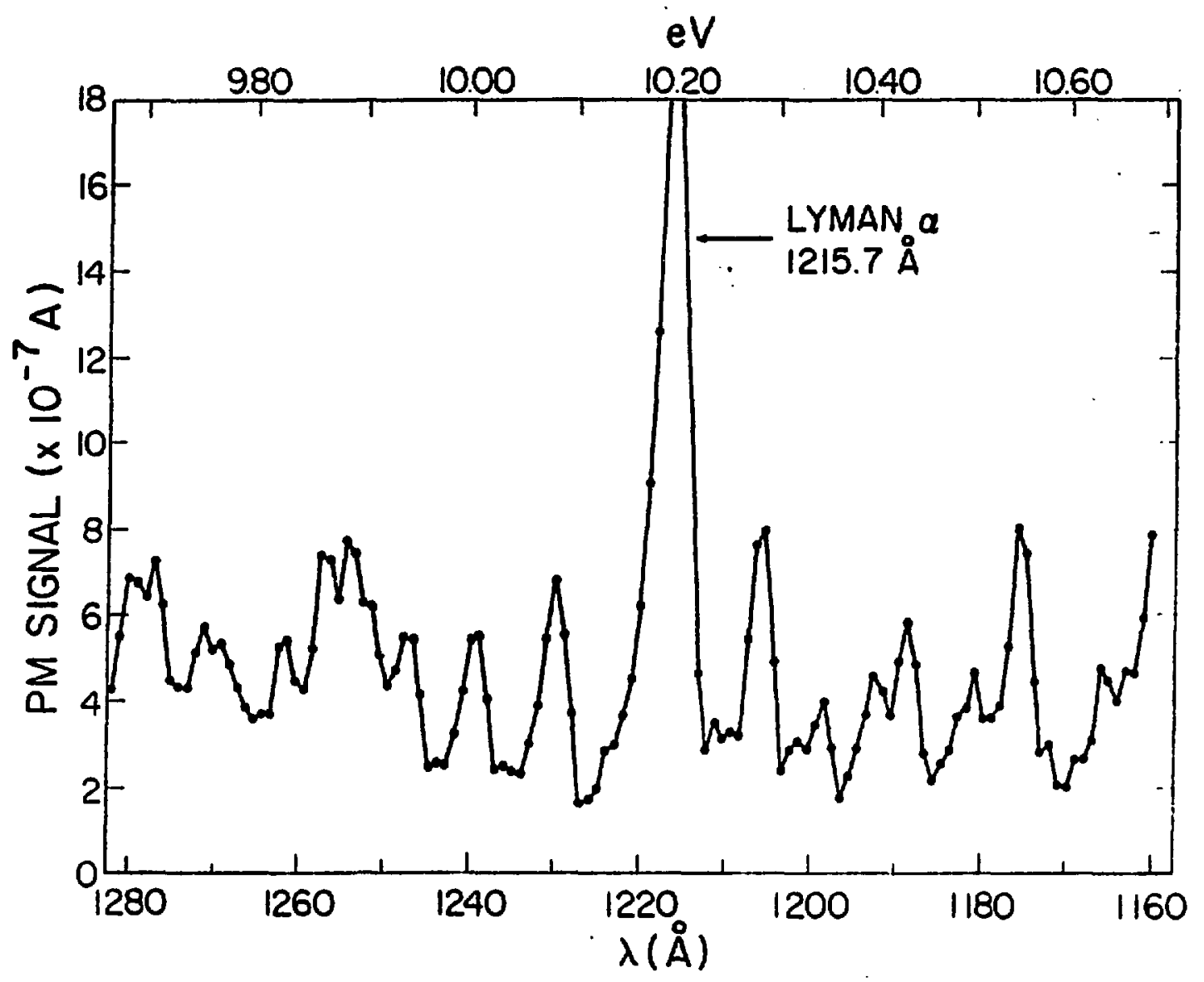

Figure 11.

$\mathrm{H}_{2}$ Spectrum Between 1160 and $1280 \AA$.

Lamp Pressure, 1000u; Discharge

Current, $300 \mathrm{~mA}$. MgF 2 - Coated

Grating, 2400 lines/mm; 200

Entrance and Exit Slits. 
Hence, the estimated photon intensity, $I_{f}$, from the sodium salicylate fluorescence may be calculated as follows :

$$
\begin{aligned}
I_{f} & =\frac{1.78 \times 10^{-8} \text { joule } / \mathrm{sec}}{4.52 \times 10^{-19} \text { joule } / \text { photon }} \\
& =3.9 \times 10^{10} \text { photons } / \mathrm{sec} .
\end{aligned}
$$

The absolute quantum efficlency of $\mathrm{NaC}_{7} \mathrm{H}_{5} \mathrm{O}_{3}$ appears to be about 65 percent. 209 As a result, the above value indicates that the Lyman $\alpha$ photon intensity, $I_{\alpha}$, striking the detector window is

$$
\begin{aligned}
I_{\alpha} & =\left(3.9 \times 10^{10} \text { photons } / \mathrm{sec}\right) \cdot \frac{1}{(.65)} \\
& =6.1 \times 10^{10} \text { photons } / \mathrm{sec} .
\end{aligned}
$$

A reasonable range for the Lyman $\alpha$ photon intensity may be established by repeating the above calculations with the reported values for minimum and maximum sensitivity for RCA 6199 phototubes. At $600 \mathrm{~V}$, these numbers are 0.16 ampere/Iumen and 4.5 amperes/Iumen respectively. 232 The corresponding estimates of maximum, $I_{\alpha, \max }$, and minimum, $I_{\alpha, m i n}$, Lyman a intensities are

$$
\begin{aligned}
& I_{\alpha, \max }=2.7 \times 10^{11} \text { photons } / \mathrm{sec} . \\
& I_{\alpha, \min }=9.4 \times 10^{9} \text { photons } / \mathrm{sec} .
\end{aligned}
$$

From Flgure 11, it is evident that the photomultiplier current output for much of the $\mathrm{H}_{2}$ spectrum lies within a factor of two of $4 \times 10^{-7}$ ampere or about $x 25$ below that at Iyman $\alpha$. Using the value for typlcal sensitivity of 
an RCA 6199 photomultiplier, a similar calculation to those above indicates that typical photon intensities are approximately $2.5 \times 10^{9}$ photons/sec. Thus, it is apparent that the overall spectral intensity obtained with the present apparatus is roughly equal to that reported by Samson (see Figure 8).209

\section{Mass Analysis and Ion Detection}

Tine basic configuration of the mass spectrometer and detector is shown in Figure 10. Mass analysis Is accomplished by a $60^{\circ}$-sector electromagnet (LASL PN\#149614) with a $17.750 "$ radius of curvature. This instrument was developed by Silvio Balestrini at Los Alamos for bomb cloud sample analysis and was inftially designed for moderate to high resolution ( 1 amu in 1000). In the present experimental arrangement, the drift tube entrance and exit slits are opened wide enough to maximize ion transmission while maintailing sufficlent resolution to cleanly separate I amu in $\therefore 0$. The magnet current is deliverad by a KEPCO Model JQE 100-5 power supply which has been set up for constant current operation by the addition of an external sensing resistor and an external current control resistor. The current output of the power supply is determined and regulated by a magnet scan unit (Model SCU-2, LASL PN\#205435). Initfal alignment of the instrument was 
achieved by various optical and mechanical methods. Final alignment was obtalned by lonlzing a beam of $\mathrm{Kr}$ atoms and tuning the magnet to the $84 \mathrm{Kr}^{+}$signal. This peak could be unambiguously identifled from the relative natural abundances of the isotopes of krypton. The magnet was shifted to adjust the radius of curvature, $r$, of ${ }^{84} \mathrm{Kr}^{+}$until the mass spectrometer equation for this Ion was satisfied. For this instrument, the mass spectrometer equation of motion is

$$
\mathrm{m} / \mathrm{e}=9.8067 \times 10^{-2} \frac{\mathrm{H}^{2}}{\mathrm{~V}}
$$

where $m$ is the ion mass in atomic mass units, $e$ is the number of electronic charges per ion, $H$ is the magnetic fleld strength in gauss and $V$ is the potential drop in volts that each ion experfences (very nearly the applied fon accelerating voltage plus the repeller voltage). This relation may be derived from the fundamental expression

$$
\frac{\mathrm{mv}^{2}}{\mathrm{r}}=\mathrm{Hev}
$$

which equates the centrifugal force of the ion $\left(\frac{m v^{2}}{r}\right)$ with the deflecting force (Hev) which acts on an ion in a magnetic field. In this expression, $m$ is the mass in grams, $H$ is in esu, e is in esu, $v$ is the fon velocity in centimeters per second and $r$ is in centimeters. By rearrangement, Equation (59) becomes

$$
v=\frac{\text { Her }}{M} \text {. }
$$


During acceleration through a potentlal of $V$ (in esu), an Ion gains kinetic energy $e V$. Provided that this energy is large compared to the initial energy of the Ion,

$$
\mathrm{eV}=\frac{\mathrm{mv} \mathrm{v}^{2}}{\mathrm{r}}=\frac{\mathrm{H}^{2} \mathrm{e}^{2} \mathrm{r}^{2}}{2 \mathrm{~m}}
$$

or

$$
m / e=\frac{H^{2} r^{2}}{2 V} .
$$

In oider to obtain $H$ in gauss, it is recessary to divide by $c^{2}$, where $c$ is the velocity of light in centimeters per second. $\checkmark$ in practical volts may be used if this expression is multiplied by 299.793. To convert from grams to amu, Avogadro's number must be included in the numerator and e ir. electronic charges may be obtalned by dividing the expression by $2.082 \times 10^{9}$. Hence

$$
\frac{m}{e}=\frac{H^{2} r^{2}}{2 V} \cdot \frac{\left(6.023 \times 10^{23}\right)\left(2.99793 \times 10^{2}\right)}{\left(2.082 \times 10^{9}\right)\left(2.998 \times 10^{10}\right)^{2}} \cdot
$$

That is,

$$
\frac{m}{e}=4.825 \times 10^{-5} \mathrm{r}^{2} \frac{\mathrm{H}^{2}}{\mathrm{~V}} .
$$

The corresponding relation for $r$ in inches is

$$
\frac{m}{e}=3.113 \times 10^{-4} \mathrm{r}^{2} \frac{\mathrm{H}^{2}}{\mathrm{~V}} \text {. }
$$

Substituting the value for the radius of curvature of the present instrument gives Equation (58).

In the alignment procedure, the applied ion accelerating voltage was measured by using a voltage 
divider and a digital multimeter. At the same time, $H$ was determined by a Harvey-Wells Model FC-502 NMR field control unit and a Hewlett-Packard Model 5300A measuring system coupled with a Model 5302A frequency counter. H In gauss may be calculated from the straight Ine relationship between the magnetic field strength and the frequency of proton resonance ( $4257.76 \mathrm{~Hz}$ /gauss). This instrumentation was retained for mass identification purposes during the whole course of the present investigation.

W1th electron bombardment, sufflcient numbers of mass selected ions are transmitted to permit detection by means of a Faraday cup arrangement or a mult1-stage electron multiplier. The current output from these devices is passed to a Keithley Model 417 picoammeter. The gain of the EMI 9642/2B 18-stage photomultiplier used In the present study has been measured as a function of negative potential at the cathode and is given in Table I. A mu metal magnetic shield is kept around the multiplier at all times. In the photoionization mode, pulse counting is performed using a PAR Model 1120 amplifier-discriminator and a PAR Model 1112 photon counter. It has proved to be necessary to employ a $20 \mathrm{db}$ attenuator between the output of the electron multiplier and the amplifier-discriminator in order to eliminate a 
Table I. Gain of EMI 9642/2B Electron Multiplier

as a Function of Cathode Potential

\begin{tabular}{|c|c|c|c|}
\hline Voltage Apolled & Ga1n & Voltage Apolied & Ga1n \\
\hline-600 & .200 & -2300 & $7.47 \times 10^{5}$ \\
\hline-700 & .986 & -2400 & $1.13 \times 10^{6}$ \\
\hline-800 & 4.26 & -2500 & $1.75 \times 10^{6}$ \\
\hline-900 & $1.73 \times 10^{1}$ & -2600 & $2.65 \times 10^{6}$ \\
\hline-1000 & $5.33 \times 10^{1}$ & -2700 & $3.97 \times 10^{6}$ \\
\hline-1100 & $1.66 \times 10^{2}$ & -2800 & $5.77 \times 10^{6}$ \\
\hline-1200 & $4.26 \times 10^{2}$ & -2900 & $3.42 \times 10^{6}$ \\
\hline-1300 & $1.08 \times 10^{3}$ & -3000 & $1.23 \times 10^{7}$ \\
\hline-2400 & $2.66 \times 10^{3}$ & -3100 & $1.68 \times 10^{7}$ \\
\hline-1500 & $5.87 \times 10^{3}$ & -3200 & $2.34 \times 10^{7}$ \\
\hline-1600 & $1.23 \times 10^{4}$ & -3300 & $3.14 \times 10^{7}$ \\
\hline-1700 & $2.41 \times 10^{4}$ & -3400 & $4.14 \times 10^{7}$ \\
\hline-1800 & $4.68 \times 10^{4}$ & -3500 & $5.28 \times 10^{7}$ \\
\hline-1900 & $8.70 \times 10^{4}$ & -3600 & $6.71 \times 10^{7}$ \\
\hline-2000 & $1.61 \times 10^{5}$ & -3700 & $3.29 \times 10^{7}$ \\
\hline-2100 & $2.74 \times 10^{5}$ & -3800 & $=.03 \times 10^{8}$ \\
\hline-2200 & $4.54 \times 10^{5}$ & -3000 & $1.23 \times: 0^{3}$ \\
\hline
\end{tabular}


troublesome background count rate ( $80-200$ counts/sec) arlsing from electronic noise. With this technique, extremely low background signals result at the expense of some $60 \%$ of the genulne signal.

It need hardly be stated that it is extremely Important to operate the mass spectrometer and detector under high vacuum conditions. A drift tube and detection chamber pressure of less than $2 \times 10^{-7}$ torr is maintained by a 4" CVC Model PMC-4 diffusion pump equipped with a Freon-cooled trap. Forepumping is accomplished by a Welch 1405 mechanlcal pump.

\section{Apparatus Performance Calculations}

The performance of the molecular beam photoIonization mass spectrometer described in the previous sections has been characterized by a number of experiments and calculations. Included in these results are estimates of beam number density (equivalent sample pressure), Ionization and collection efflclencles for electron Impact and Ionization and collection efficiencles for photolonization.

A molecular beam of kryoton was used to calculate a typlcal equivalent sample pressure for the present system. As a first step, the ionization chamber diffusion pump was throttled to permit the ion current measuremert for quasi-static sample. zrior to sample 
Introduction, the main chamber pressure was observed to be $3.4 \times 10^{-7}$ torr. With a nozzle source pressure of 38 torr $\mathrm{Kr}$ and the beam flag in, the pressure in this cavity rose to $1.0 \times 10^{-6}$ torr. In other words, approximately $6.6 \times 10^{-7}$ torr $\mathrm{Kr}$ was added to the system as measured by a standard ion gauge. Near static conditions were confirmed by the fact that opening and closing the beam flag had only a minor effect on the Ionization chamber pressure. With the flag closed, the current output of the electron multiplier at $1500 \mathrm{~V}$ due to ${ }^{84} \mathrm{Kr}^{+}$was $1.56 \times 10^{-9}$ amperes. Now, the natural abundance of ${ }^{84} \mathrm{Kr}$ is $56.9 \%$ and the concentrations of $\mathrm{Kr}_{\mathrm{n}}$ may be expected to be negliglble under these sample conditions. Thus, the pressure rise due to ${ }^{84} \mathrm{Kr}$ was $\left(6.6 \times 10^{-7}\right.$ torr $) \cdot(.569)$ or $3.76 \times 10^{-7}$ torr. This number must be multiplied by .538 to account for ion gauge callbration. 233 Consequently, the true pressure rise due to ${ }^{84} \mathrm{Kr}$ was $\left(3.76 \times 10^{-7}\right.$ torr $) \cdot(.538)$ or $2.02 \times 10^{-7}$ torr. The corresponding concentration of ${ }^{84} \mathrm{Kr}$ in molecules per $\mathrm{cm}^{3}$ may be estimated from Loschmidt's number, $n_{0}$. That is,

$$
n_{0}=2.687 \times 10^{19} \text { molecules } / \mathrm{cm}^{3} \text { at } 760 \mathrm{~mm} \mathrm{Hg} \text {. }
$$

Hence, the background concentration of ${ }^{84} \mathrm{Kr},\left[{ }^{84} \mathrm{Kr}\right]_{\mathrm{b}}$, 
from this experiment is given by

$$
\begin{aligned}
{\left[^{84} \mathrm{Kr}\right]_{\mathrm{b}} } & =\left(2.687 \times 10^{19} \frac{\text { molecules }}{\mathrm{cm}^{3}}\right) \frac{2.02 \times 10^{-7} \mathrm{~mm} \mathrm{Hg}}{.760 \times 10^{3} \mathrm{~mm} \mathrm{Hg}} \\
& =7.14 \times 10^{9} \text { molecules } / \mathrm{cm}^{3} .
\end{aligned}
$$

Assuming that the mass spectrometer collection efficiency Is the same for both free jet and quasi-static samples, it is possible to derive the $\mathrm{Kr}$ nozzle beam number density by measuring the appropriate electron multiplier signal for $84 \mathrm{Kr}^{+}$. With identical electron gun conditions, the maximum $84 \mathrm{Kr}^{+}$picoammeter current reading as a function of nozzle source pressure was $7.5 \times 10^{-11}$ ampere using the Faraday cup or $4.4 \times 10^{-7}$ ampere from the electron multiplier at $1500 \mathrm{~V}$. This reading was observed at a stagnation pressure of 108 torr $\mathrm{Kr}$. The ${ }^{84} \mathrm{Kr}$ molecular beam number density, $\left[{ }^{84} \mathrm{Kr}\right]_{\mathrm{m}}$, may then be calculated from the relation

$$
\begin{aligned}
{\left[^{84} \mathrm{Kr}\right]_{\mathrm{m}} } & =\left[{ }^{84} \mathrm{Kr}\right]_{\mathrm{b}} \cdot \frac{4.4 \times 10^{-7} \text { ampere }}{1.56 \times 10^{-9} \text { ampere }} \\
& =2.01 \times 10^{12} \text { molecules } / \mathrm{cm}^{3} .
\end{aligned}
$$

The corresponding equivalent sample pressure for ${ }^{84} \mathrm{Kr}, \mathrm{P}_{84} \mathrm{Kr}$, is given by

$$
\begin{aligned}
& \mathrm{P}_{84 \mathrm{Kr}}=\left(2.01 \times 10^{12 \mathrm{molecules}} \mathrm{cm}^{3}\right) \cdot \frac{760 \mathrm{~mm} \mathrm{Hg}}{2.687 \times 10^{19} \frac{\mathrm{molecules}}{\mathrm{cm}^{3}}} \\
& =5.69 \times 10^{-5} \text { torr. }
\end{aligned}
$$


The total monomer equivalent sample pressure, $P_{K r}$, is

$$
\begin{aligned}
P_{\mathrm{Kr}} & =\frac{5.69 \times 10^{-5} \text { torr }}{0.569} \\
& =1.0 \times 10^{-4} \text { torr. }
\end{aligned}
$$

Under similar source conditions, fon signals for other compounds do not vary greatly from those mentioned above. Therefore, sample pressures of about $10^{-4}$ torr may be considered typical for the present experimental design.

Using the ${ }^{84} \mathrm{Kr}$ beam number density in confunction with the measured electron trap current and various other constants for the ionization process, the electron bombardment ionization efficiency, I.E. (IB), and mass spectrometer collection efficiency, C.E. (EB), may be estimated. A reasonable value for the $\mathrm{Kr}$ monomer beam velocity is $5 \times 10^{4} \mathrm{~cm} / \mathrm{sec} .{ }^{234}$ Hence, the ${ }^{84} \mathrm{Kr}$ beam flux $\Phi_{84} \mathrm{Kr}$, may be calculated as follows:

$$
\begin{aligned}
\Phi_{84 \mathrm{Kr}} & =\left(2.01 \times 10^{12} \frac{\text { molecules }}{\mathrm{cm}^{3}}\right)\left(5 \times 10^{4} \mathrm{~cm} / \mathrm{sec}\right) \\
& =1.0 \times 10^{17} \frac{\text { molecules }}{\mathrm{cm}^{2} . \mathrm{sec}} .
\end{aligned}
$$

The current output of the ionizer electron collector in the above determinations was $1 \mathrm{~mA}$ or $6.24 \times 10^{15} \frac{\text { electrons. }}{\text { sec. }}$ The latter quantity is taken to be the number of electrons avallable for ionization per second, $I_{e}$. An estimate of the ionization cross section, $\sigma_{e}$, for $\mathrm{Kr}$ by $50 \mathrm{eV}$ electron 
Impact may be derived from the data of Beran and Kevan. 235 By IInear Interpolation, $\sigma_{e}$ is approximately $4.6 \times 10^{-16} \mathrm{~cm}^{2}$. Assuming that the beam area, A, facing the electron flow is $0.1 \mathrm{~cm}^{2}$ and also that the electrons are effective for Ionization over a path length, $d$, of $1 \mathrm{~cm}$, the number of lons produced per second, $\mathrm{N}_{8} \dot{4}_{\mathrm{Kr}}^{+}$, may be calculated as follows :

$$
\begin{aligned}
\mathrm{N}_{8} \stackrel{4}{\mathrm{Kr}}_{\mathrm{r}} & =I_{\mathrm{e}}\left[{ }^{84} \mathrm{Kr}\right]_{\mathrm{m}} \sigma_{e} \mathrm{~d} \\
& =\left(6.24 \times 10^{15} \mathrm{sec}^{-1}\right) \cdot\left(2.01 \times 10^{12} \mathrm{~cm}^{-3}\right) \cdot\left(4.6 \times 10^{-16} \mathrm{~cm}^{2}\right) \cdot(1 \mathrm{~cm}) \\
& =5.77 \times 10^{12} \mathrm{sec}^{-1} .
\end{aligned}
$$

As mentioned previously, the observed ${ }^{84} \mathrm{Kr}{ }^{+}$ion current was $7.5 \times 10^{-11}$ amperes. Thus, the ${ }^{84} \mathrm{Kr}^{+}$detection rate, $\mathrm{D}_{84}^{+} \mathrm{Kr}$, is equal to $4.68 \times 10^{8} \mathrm{sec}^{-1}$. From these quantities, the net efficiency, N.E. (EB), as well as I.E. (EB) and C.E. (EB) may be computed:

$$
\begin{aligned}
& \mathrm{N} . \mathrm{E} \cdot(\mathrm{EB})=\frac{\mathrm{D}_{8}^{+}}{\mathrm{A} \cdot \Phi \mathrm{Kr}}=\frac{4.68 \times 10^{8} \mathrm{sec}^{-1}}{\left(0.1 \mathrm{~cm}^{2}\right)\left(10^{17} \mathrm{~cm}^{-2} \mathrm{sec}^{-1}\right)} \\
& =4.68 \times 10^{-8} \\
& I . E .(E B)=\frac{N^{\$} 84 \mathrm{Kr}}{A \cdot \Phi 84 \mathrm{Kr}}=\frac{5.77 \times 10^{12} \mathrm{sec}^{-1}}{\left(0.1 \mathrm{~cm}^{2}\right)\left(10^{17} \mathrm{~cm}^{-2} \mathrm{sec}^{-1}\right)} \\
& =5.77 \times 10^{-4} \\
& \text { C.E. }(E B)=\frac{N \cdot E \cdot(E B)}{I \cdot E \cdot(E B)}=\frac{D^{+} 4_{\mathrm{Kr}}}{\mathrm{N}_{84}^{+} \mathrm{Kr}} \\
& =8.11 \times \geq 0^{-5} \text {. }
\end{aligned}
$$


Because of the many assumptions involved in these calculations, the true values for N.E. (EB), I.E. (EB) and C.E. (EB) may. differ substantially from the numbers above. In fact, it seems almost certain that the mass spectrometer collection efficiency is higher than $8 \times 10^{-5}$ since the observed mass peaks are quite sharp when the instrument is operated at high resolution. Moreover, further opening of the drift tube slits produces only a very small enhancement of the signal intensity (i.e., there are probably negligible losses at the sIIts).

It is interesting to compare the foregoing electron impact results for $84 \mathrm{Kr}$ with similar calculations for the photolonization of acetone at Lyman $\alpha$. For these computations, it is necessary to make several preliminary assumptions and estimates. Since the beam velocity of $\mathrm{CH}_{3} \mathrm{COCH}_{3}$ was not measured in the present study, a value of $5 \times 10^{4} \mathrm{~cm} / \mathrm{sec}$ will be employed once again. AIso, a specific measurement of acetone equivalent sample pressure was not performed; however, a comparison of electron bombardment ion signals for $\mathrm{CH}_{3} \mathrm{COCH}_{3}{ }^{+}$and ${ }^{84} \mathrm{Kr}^{+}$indicates that this parameter IIkeIy falls in the range of $10^{-5}-10^{-4}$ torr. The beam volume which interacts with the lonizing medium is taken to be the same as before $\left(0.1 \mathrm{~cm}^{3}\right)$. The 1215.7 photon 
intensity, $I_{\alpha}$, has been calculated previousiy (see Equation (55) in Section III.B.). Finally, it is necessary to establish a reasonable value for the photoionization cross section of acetone, $\sigma_{A C}$, at Lyman $\alpha$. Beran et. al. have determined that $\sigma_{A c}$ is 3.13 times larger than the cross section for NO at 11.6-11.8 ev. ${ }^{236}$ Now, the photolonization cross section for NO in this energy range is apparently about $3 \times 10^{-18} \mathrm{~cm}^{2} .12$ consequently, $\sigma_{\text {Ac }}$ at $11.6-11.8 \mathrm{eV}$ is approximately $9.4 \times 10^{-18} \mathrm{~cm}^{2}$. In view of the fact that the photoion yield curve of acetone rises slightly in going from this region to Lyman $\alpha, 23710^{-17} \mathrm{~cm}^{2}$ is an acceptable $\sigma_{A c}$ for these calculations.

Assuming an equivalent acetone sample pressure, ${ }^{2} \mathrm{Ac}$, of $10^{-4}$ torr, the $\mathrm{CH}_{3} \mathrm{COCH}_{3}$ beam number density, [AC], may be calculated as follows:

$$
\begin{aligned}
{[A] c] } & =P_{A c} \cdot \frac{2.687 \times 10^{19} \text { molecules } / \mathrm{cm}^{3}}{.760 \times 10^{3} \mathrm{~mm} \mathrm{Hg}} \\
& =3.5 \times 10^{12} \mathrm{molecules} / \mathrm{cm}^{3} .
\end{aligned}
$$

The acetone beam flux, $\Phi_{A C}$, is then estimated by the relation

$$
\begin{aligned}
\Phi_{A C} & =\left(3.5 \times 10^{12} \frac{\text { molecules }}{\mathrm{cm}^{3}}\right) \cdot\left(5 \times 10^{4} \frac{\mathrm{cm}}{\mathrm{sec}}\right) \\
& =1.75 \times 10^{17} \frac{\text { molecules }}{\mathrm{cm} 2 \mathrm{sec}} .
\end{aligned}
$$


The number of acetone ions produced per second, $\mathrm{N}_{\mathrm{Ac}}^{+}$, may be computed from the following expression:

$$
\begin{aligned}
N_{A C}^{+} & =I_{\alpha}[A C] \sigma_{A C}{ }^{\alpha} \\
& =\left(6.1 \times 10^{10} \mathrm{sec}^{-1}\right) \cdot\left(3.5 \times 10^{12} \mathrm{~cm}^{-3}\right) \cdot\left(10^{-17} \mathrm{~cm}^{2}\right) \cdot(1 \mathrm{~cm}) \\
& =2.1 \times 10^{6} \mathrm{sec}^{-1} .
\end{aligned}
$$

The $\mathrm{CH}_{3} \mathrm{COCH}_{3}{ }^{+}$signal for the oresent aoparatus is typically 400 counts/second at Lyman $\alpha$. However, oniy $40 \%$ of the genuine ion count is detected when the $20 \mathrm{db}$ attenuator is employed in the data collection electronics. Hence, the appropriate acetone ion detection rate, $\mathrm{D}_{\mathrm{Ac}}^{+}$, Is $1000 \mathrm{sec}^{-1}$. The net photoionization efficiency N.E. (hv), and the ionization and collection efficlencies, I.E. (hv) and C.E. (hv), may be calculated as before:

$$
\begin{aligned}
\text { N.E. }(h v) & =\frac{D_{A C}^{+}}{A \cdot \Phi_{A C}}=\frac{10^{3} \mathrm{sec}^{-1}}{\left(0.1 \mathrm{~cm}^{2}\right) \cdot\left(1.75 \times 1017 \mathrm{~cm}^{-2} \mathrm{sec}^{-1}\right)} \\
& =5.7 \times 10^{-14}
\end{aligned}
$$

I.E. $(h v)=\frac{N_{A c}^{+}}{A \cdot \Phi_{A C}}=\frac{2.1 \times 10^{6} \mathrm{sec}^{-1}}{\left(0.1 \mathrm{~cm}^{2}\right) \cdot\left(1.75 \times 10^{-17} \mathrm{~cm}^{-2} \mathrm{sec}^{-1}\right)}$

$$
=1.2 \times 10^{-10}
$$

$$
\begin{aligned}
C . E .(h v) & =\frac{N \cdot E \cdot(h v)}{I \cdot E \cdot(h y)}=\frac{D_{A C}^{+}}{N_{A C}^{+}} \\
& =4.8 \times 10^{-4} .
\end{aligned}
$$

If $P_{A C}$ is taken to be $10^{-5}$ torr, C.E. (hv) is equal to $4.8 \times 10^{-3}$. In any case, the estimated mass spectrometer collection epiciency por photoionization is considerajiy 
higher than the ${ }^{84} \mathrm{Kr}$ electron bombardment result. Nevertheless, this value is stili far below the fon transmission efflclencies clalmed by Chupka and Berkowttz 55,140 for a similar instrument. The reason for this discrepancy is not readily apparent.

\section{E. Data Collection Procedures}

The vacuum pumps on the nozzle exhaust chamber and second differential molecular beam pumping chamber are not equipped with Ilquid nitrogen traps and are operated only during the course of an experimental run. Consequentiy, the first step in preparing the apparatus for a photolonization efficiency curve measurement is to activate these pumps. Once the system achieves a sultable vacuum, the sample compound may be introduced into the gas mantfold and expanded through the nozzle aperture. This gas flow is regulated according to the approximate stagnation pressure required and at least 15-30 minutes are provided for the expansion to stabilize. Also, a flow of $\mathrm{H}_{2}$ lamp gas is inftiated such that a discharge capiliary pressure of $600-1000 \mu$ is obtalned. Improved lamp stability has been found to result from operating the discharge for as much as one hour before beginning the experiment. Typlcal power supply conditions are $1500 \mathrm{~V}$ and $450 \mathrm{~mA}$. At the same time, the remaining electronic Instruments are turned on and allowed to varm up. A 
schematic representation of much of the operating and data collection instrumentation is given in Figure 12. The magnet is roughly tuned to the desired $\mathrm{m} / \mathrm{e}$ position using the NMR field control unit and frequency counter arrangement described in section III.C. Electron bombardment or photolonization at an intense spectral line (e.g., Lyman $\alpha, 1160 \AA$, etc.) Is used to 1dentify and maximize the mass peak corresponding to the specie of interest. Ion transmission is optimized by adfusting the repeller voltage (controlied by the Model ER-I emission regulator) as well as the voltages applied to the dalr of quadrubole lenses. When fine tuning of the ion siznal is accomplished, the resonance pattern displayed by the MMR field control unit is centered on the oscliloscope scale. This pattern is carefully monitored in order to detect and prevent a mass drift.

The photolonization efficiency is determined $b y$ the ratio of ion signal to photon signal as a function 0 :? wavelength (or ohoton energy). At each point, mass resolved ion counts are accumulated for a soesified Deriod of time and the total count is recorded. The chotomultiplier current reading, which is proportional to the monochromatic light intensity, is also recorded. Voltages of about $-3500 \mathrm{~V}$ and $-600 \mathrm{~V}$ are typlcally applied to the 


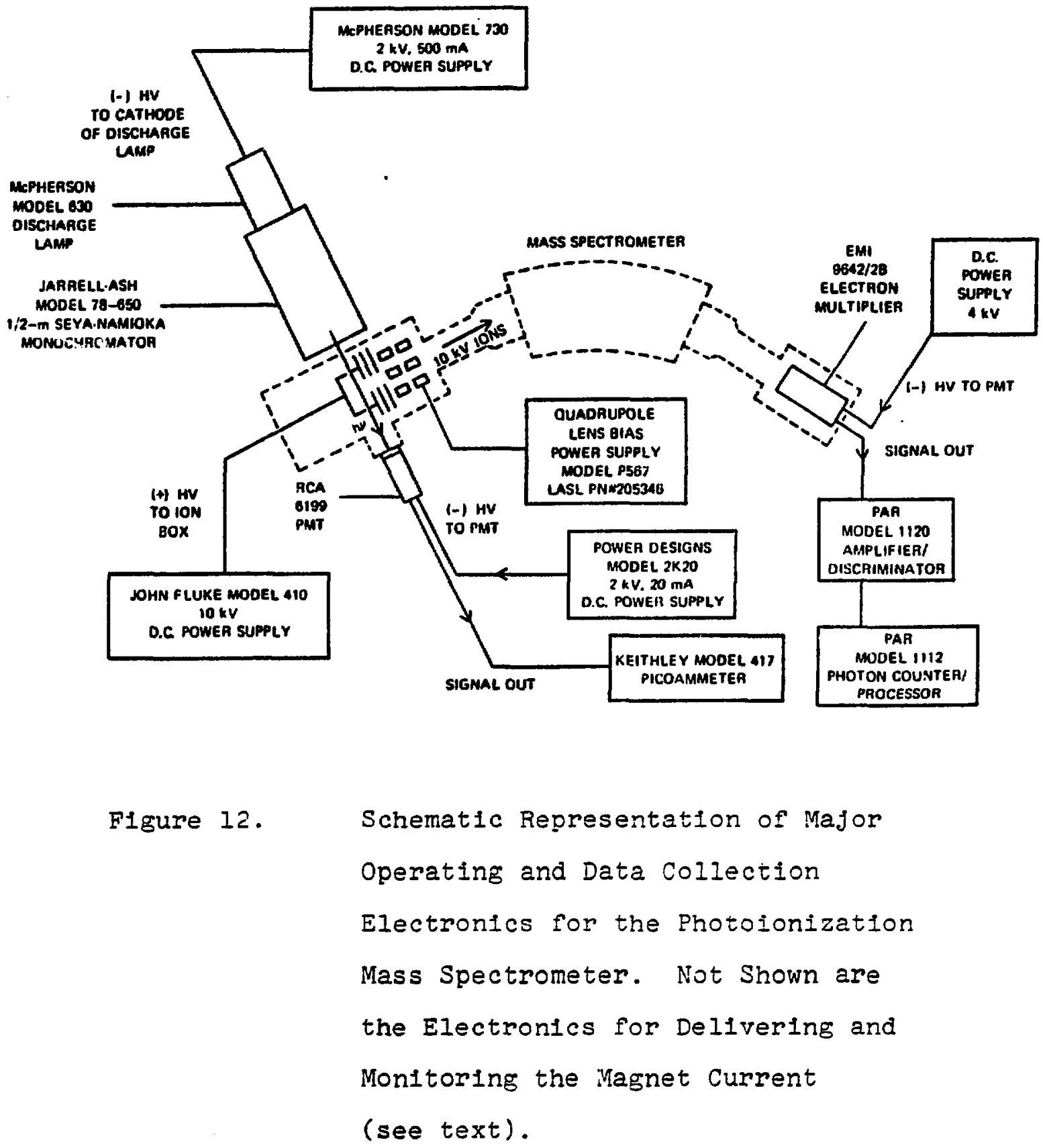


electron multiplier and RCA 6199 photomultiplier tube, respectively. The lamp intensity has been found to remain stable to $\pm 2 \%$ at each wavelength. The $\mathrm{H}_{2}$ spectrum is scanned by manually advancing the monochromator. In the present study, data has generally been taken at $1-2.5 \AA$ intervals. Inasmuch as a 2400 lines/mm grating is used in confunction with a grating drive mechanism designed for 1180 Iines $/ \mathrm{mm}$, it is necessary to establish wavelength calibration with the known hydrogen atom and molecule emission lines. 209,238 It is important to make background corrections in both the photoion and photon signals. Fortunately, these effects are quite small. A particularly low lon counting background rate can be achleved if all ion gauge fllaments are turned off. Under these conditions, the ion count with the molecular beam flagged is typically about 0.02-0.1 count/sec. Thus, reproducible ion signals of 0.03-0.05 count/sec are detectable. Background counts are accumulated after every flve or six data points in order to carefully monitor this factor. In addition, a reference data point is occasionally repeated to check for small changes in beam intensity, collection efficiency, etc. Since a holographically recorded diffraction grating is used, stray light levels are expected to be substan- 
tially reduced.126 Consequently, the photon signal correction should very nearly be the photomultiplier dark current. As mentioned previously, it is possible to reproduce in substantial deta1l the $\mathrm{H}_{2}$ spectrum given by Samson. 209 


\section{RESULTS AND DISCUSSION}

A. Preliminary Observations with Electron Impact Prior to the full assembly and optimization of the monochromatic vacuum ultraviolet photon source, a considerable number of parent, cluster and fragment ions were seen using electron bombardment on various compounds in molecular beams. As Indicated in Section III.D., measurements on rare gas specles were made in order to test and characterize the sampling system and mass spectrometer. In this regard, the observed similarity of the nozzle stagnation pressure dependence of rare gas monomer and dimer ion signal intensities in the present study as compared to the room temperature skimmer and endwall results of Bailey et. al. 199 (see Figure 7) is of particular interest. A number of other compounds were studied with the object of determining the feasibility of their investigation by photolonization. A summary of the ions detected and additional relevant comments are given in Table II.

B. Photoionization of Acetone and Acetone-d 6 The application of high resolution molecular beam-photolonization mass spectrometry to complex organic molecules remains a largely unexplored possibility. 
Table II. Summary of Electron Bombardment Results

for Various Compounds in Molecular Beams

Molecular Beam Constiluents

Argon, Krypton, Xenon

Annonia

Carbon Dloxide

Acetone

Proptonaldehyde

Gyclopentane

(j)ygen

Water

Nitric oxide and carbon Dioxide

Carbon Dloxide and Water
Ions Observed

$\mathrm{Kr}^{+}, \mathrm{Kr}_{2}^{+}, \mathrm{Xe}^{+}, \mathrm{Xe}_{2}^{+}$,

$A r^{+}, \mathrm{Ar}_{2}^{+}, \mathrm{KrAr}^{+}, \mathrm{XeAr}^{+}$

$\mathrm{NI}_{3}^{+}, \mathrm{NH}_{4}^{+}, \mathrm{NH}_{2}^{+}$

$\left(\mathrm{NII}_{3}\right)_{2}^{+},\left(\mathrm{NII}_{3}\right)_{3}^{+}$.

$\left(\mathrm{NII}_{3}\right)_{n} \mathrm{H}^{+}, n=2-15$

$\mathrm{CO}_{2}{ }^{+},\left(\mathrm{CO}_{2}\right)_{n}{ }^{+}, \mathrm{n}=2-7$

$\mathrm{Cll}_{3} \mathrm{COCH}_{3}^{+}, \mathrm{Cll}_{3} \mathrm{CO}^{+}$

$\left(\mathrm{Cll}_{3} \operatorname{coch}_{3}\right)_{n}^{+}, n=1-6$

$\left(\mathrm{CH}_{3} \mathrm{COCH}_{3}\right) \mathrm{H}^{+}, \mathrm{n}=1-6$

$\mathrm{CH}_{3} \mathrm{CH}_{2} \mathrm{CllO}^{+}$and fragments

$\mathrm{C}_{5} \mathrm{H}_{10}+$ and fragments.

$\mathrm{O}_{2}^{+},\left(\mathrm{o}_{2}\right)_{2}^{+}$

$\mathrm{H}_{2} \mathrm{O}^{+}, \mathrm{Oll}^{+},\left(\mathrm{H}_{2} \mathrm{O}\right)_{n}^{+}, \mathrm{n}=2-5$

$\left.\left(H_{2}\right)_{n}\right)^{+}, n=2-5$

$\mathrm{NO}^{+}, \mathrm{CO}_{2}^{+},(\mathrm{NO})_{2}^{+}, \mathrm{NO}^{+} \cdot \mathrm{CO}_{2}$,

$\left(\mathrm{CO}_{2}\right)_{2}^{+},\left(\mathrm{CO}_{2}\right)_{3}^{+}$

$\mathrm{H}_{2} \mathrm{O}^{+}, \mathrm{H}_{3} \mathrm{O}^{+},\left(\mathrm{II}_{2} \mathrm{O}\right)_{2}^{+},\left(\mathrm{II}_{2} \mathrm{O}\right)_{2} \mathrm{H}^{+}$,

$\mathrm{CO}_{2}^{+},\left(\mathrm{II}_{2} \mathrm{O}\right)_{3}^{+},\left(\mathrm{II}_{2} \mathrm{O}\right)_{3} \mathrm{H}^{+}$,

$\mathrm{Co} \cdot \mathrm{I}_{2} \cdot \mathrm{H}_{2} \mathrm{O}^{+}, \mathrm{CO} \mathrm{O}_{2} \cdot \mathrm{H}_{3} \mathrm{O}^{+},\left(\mathrm{CO}_{2}\right)_{2}{ }^{+}$

\section{Comments}

Source pressure dependence of monomer and dimer algnal intensity similar to warm skimmer results of reference 199.

Extens I ve fragmentation: $\left(\mathrm{NH}_{3}\right)_{\mathrm{n}-1} \mathrm{H}^{+}$ slgnal Intensities $\times 25-50$

larger than those for $\left(\mathrm{NH}_{3}\right)_{n}$ wth

100-140 eV electron impace.

See Section IV.B.

Extensive fragmentation; no dimer Ions seen.

Substantial fragmentation; no dimer lons seen.

Particularly large signal correspond1uge to $\left(\mathrm{H}_{2} \mathrm{O}\right)_{3} \mathrm{Il}^{+}$observed.

Mixed dimer signal small.

M1xed dimer signals small. 
Except for monomer and cluster studies of ethylene 82 and smaller alcohols, 91,92 this method has generally been confined to experiments involving parent molecules with five atoms or less. ${ }^{79-81,83-90}$ Departing from this trend, the present study is in part concerned with the photolonization of several species produced In supersonic nozzle expansions of acetone and acetone- $d_{6}$ vapor. As the simplest aliphatic ketone, the lonization and dissoclative properties of acetone are of significant interest as being representative of a slzeable homologous class of substances. Consequently, this compound has been extensively studied by various methods including electron impact, 239-254 photoionization, $12,36,45,50,58,237,255-257$ photoelectron spectroscopy, 258-271 photoelectron-photolon colncldence spectroscopy, 272,273 vacuum ultraviolet absorption spectroscopy, ${ }^{11}$ Penning Lonization, 274,275 charge transfer spectra 276 and electron energy loss spectroscopy. 277 The more recent of these experiments have produced fairly consistent firs and higher Ionlzation potential values for the parent species and appearance potential values for the acetyl fragment ion. Ittle additional ionic state information has been determined, however. 
Using nozzle beam sampling techniques, the photolonization efficiency curves for $\mathrm{CH}_{3} \mathrm{COCH}_{3}^{+}$and $\mathrm{CH}_{3} \mathrm{CO}^{+}$have been measured more precisely in order to obtain such data as low energy ionic vibrational frequencles, Franck-Condon factors for direct Ionization and effects of thermal relaxation on threshold values. Photolon yleld curves have been determined for $\mathrm{CD}_{3} \mathrm{COCD}_{3}{ }^{+}$and $\mathrm{CD}_{3} \mathrm{CO}^{+}$as well. In addition, photolonization mass spectrometric measurements of $\left(\mathrm{CD}_{3} \mathrm{COCD}_{3}\right)_{2}^{+},\left(\mathrm{CH}_{3} \mathrm{COCH}_{3}\right) \mathrm{n}^{+}, \mathrm{n}=2-4$ and $\left(\mathrm{CH}_{3} \mathrm{COCH}_{3}\right) \cdot \mathrm{CH}_{3} \mathrm{CO}^{+}$ are reported.

\section{Experimental}

For the photolonization study of acetone, an Intense supersonic expansion was produced by seeding $\mathrm{CH}_{3} \mathrm{COCH}_{3}$ vepor at $20-25^{\circ} \mathrm{C}$ in $\mathrm{H}_{2}$. Total stagnation pressures were varled between 350-750 torr in orcier to maximize the concentration of siven components in separate experiments. Inasmuch as some enhancement of cluster ion Intensities relative to the parent could be achieved by increasing the beam stagnation pressure, the ratio of $\mathrm{H}_{2}$ to acetone used was generally higher for the polymer studies. However, the functional dependence of cluster fon signal and $\mathrm{H}_{2}$ source pressure was not found to be particularly strong. Considerable are was taken to maintain a coristant acetore source 
temperature and total stagnation pressure throughout each run. Because mass analysis was employed during the whole course of the present study, Mallinclcrodt Spectrar grade acetone (99.5\% minimum purity) was used without further purification; 99.5 atom \% $D$ acetone-d 6 , obtained from Aldrich Chemical Co., Inc., was used directly for the isotope work.

Two different monochromator entrance and exit slit widths were used, depending upon the intensity of the specie investigated. Operating conditions for the discharge lamp were typically 0.3 torr $H_{2}$ pressure and $450 \mathrm{~mA}$ discharge current. The acetone and acetone-d 6 monomer studies were performed at an estimated resolution of $1.5 \&$ corresponding to the use of $200 \mu$ slits. Counts were collected for 500-600 seconds per data point at approximately $I$ intervals. Counting rates in the region $1240-1280 \AA$ varied from about 15 counts/second to approximately 0.5 count/second at threshold (at Lyman $\alpha$, counting rates could be varied from 200 counts/second to 650 counts/second, depending upon lamp pressure and nozzle expansion conditions). For cluster and fragment Ion studies, $500 \mu$ monochromator entrance and exit slits were used, resulting in a resolution of approximately $3.75 \AA$ or $30 \mathrm{met}$ at $1200 \AA$. Readings were taken at 1.5-2.5 A intervals fon 500-300 seconds per data point. 
Typlcal counting rates ranged from 0.03 count/second to 5 counts/second. In each run, a reference polnt was repeated periodically to account for any drift in th: mass peak, co+lection efficlency, etc.

\section{Results}

A partial mass spectrum for an acetone-i. 2 molecular beam ( 600 torr total stagnation pressure) was obtained using $100 \mathrm{eV}$ electron bombardment. Signals corresponding to acetone monomer and cluster lons were observed at $\mathrm{m} / \mathrm{e} 58,116,174,232,290$, and 348 . Fragment lons at $\mathrm{m} / \mathrm{e} 43$ and 101 were seen in concentrations $\times 3-\times 4$ those of the monomer and dimer ion, respectively. In addition, small peaks corresponding to lons of the form $\left(\mathrm{CH}_{3} \mathrm{COCH}_{3}\right)_{\mathrm{n}^{\mathrm{H}^{+}}}, \mathrm{n}=1-6$, were observed at $\mathrm{m} / \mathrm{e} 59,117,175,233,291$, and 349. These lons presumably orlfinate from high energy fragmentation of cluster spectes or fon-molecule reactions involving some of the numerous parent, cluster, and fragment ions iormed; 1.e., $\left(\mathrm{CH}_{3} \mathrm{COCH}_{3}\right)_{\mathrm{n}}^{+},\left(\mathrm{CH}_{3} \mathrm{COCH}_{3}\right)_{\mathrm{n}} \cdot \mathrm{CH}_{3} \mathrm{CO}^{+}, \mathrm{CH}_{3}^{+}$, $\mathrm{H}_{2}^{+}, \mathrm{H}^{+}$, etc. Protonated acetone and acetone cluster fons have been previously reported in field ionization 278 and lon-molecule reaction $279-285$ studies.

In contrast, the photolonization mass spectrum for the acetone-H $\mathrm{H}_{2}$ beam was markedly less complex in the 
region 1100-1300 A. At 500 torr total stagnation pressure, ions of the form $\left(\mathrm{CH}_{3} \mathrm{COCH}_{3}\right)_{n}{ }^{+}, n=1-4$, were observed at Lyman $\alpha$ in the approximate ratio 100:40:5:3. Signals at $\mathrm{m} / \mathrm{e} 59,117,175$, etc. were seen only in those concentrations which would be expected on the basis of natural isotopic abundances of ${ }^{13} \mathrm{C}$ and $\mathrm{D}$. At $1160 \AA$, the $\mathrm{CH}_{3} \mathrm{CO}^{+}$and $\left(\mathrm{CH}_{3} \mathrm{COCH}_{3}\right) \cdot \mathrm{CH}_{3} \mathrm{CO}^{+}$fons (m/e 43 and 101) could be seen in very low concentrations. The relative simpliclty of the photolorization spectra was taken as strong evidence that only simple ionization and fragmentation processes occur. 284

Photoion yleld curves obtained in the present work are shown in Flgures 13-20. With the exception of the acetone tetramer and acetcne-d $d_{6}$ dimer, each ion was investigated at least twice. The spectra were found to be reproducible in substantial detail. The measured ionlzation potentials (IP) and appearance potentials (AP) of the various species are given in Table III, along with Ionic vibrational structure information which has been derived from the experimental curves.

\section{Discussion}

a. Acetone and acetone-do monomer ions The photolonization efficiency curves for acetone and acetone-de parent lons are characterized 


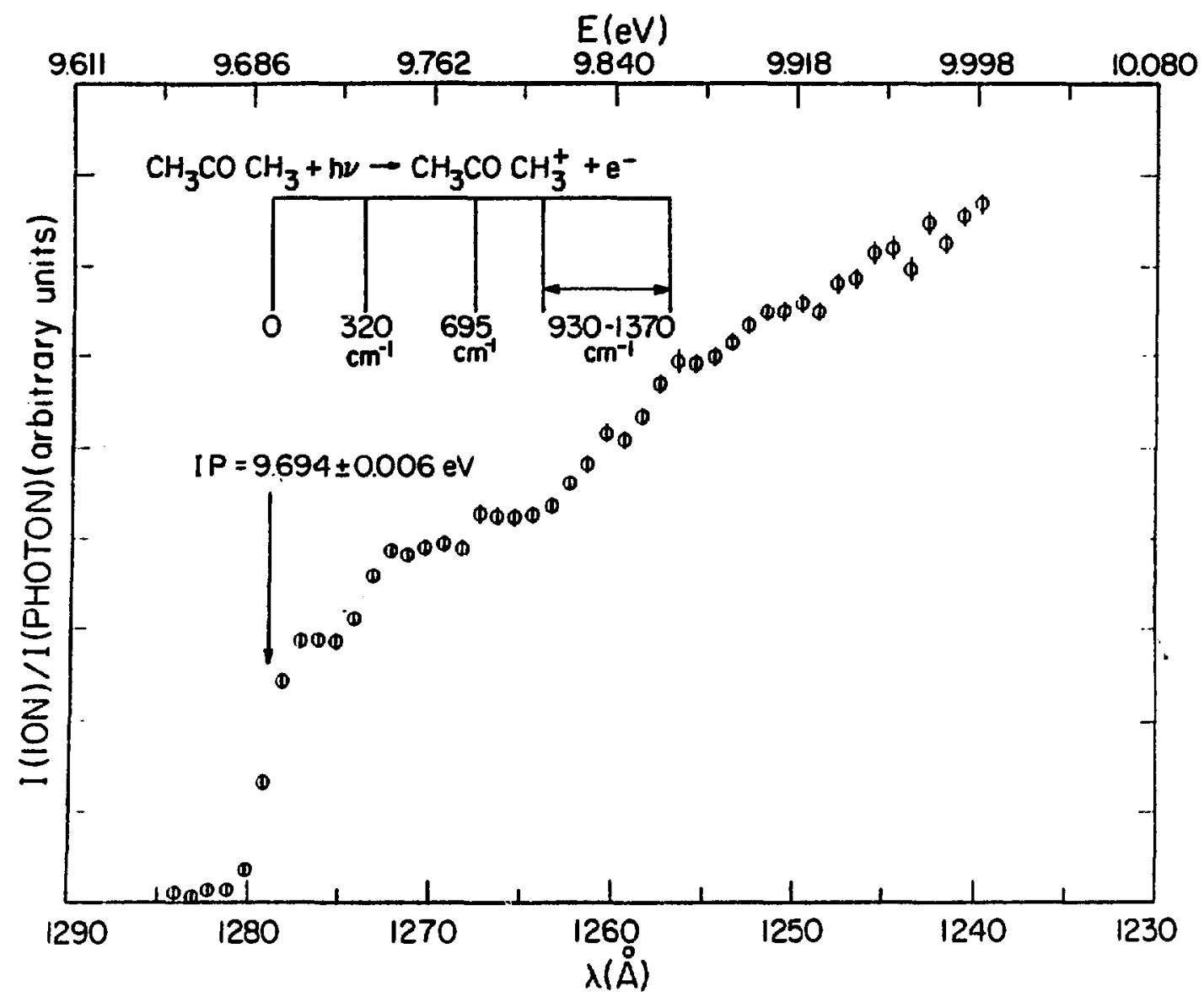

F1gure 13.

Photoion Yleld Curve for $\left(\mathrm{CH}_{3} \mathrm{COCH}_{3}\right)^{+}$ from 1240 to $1285 \stackrel{\circ}{\text {. }}$. 


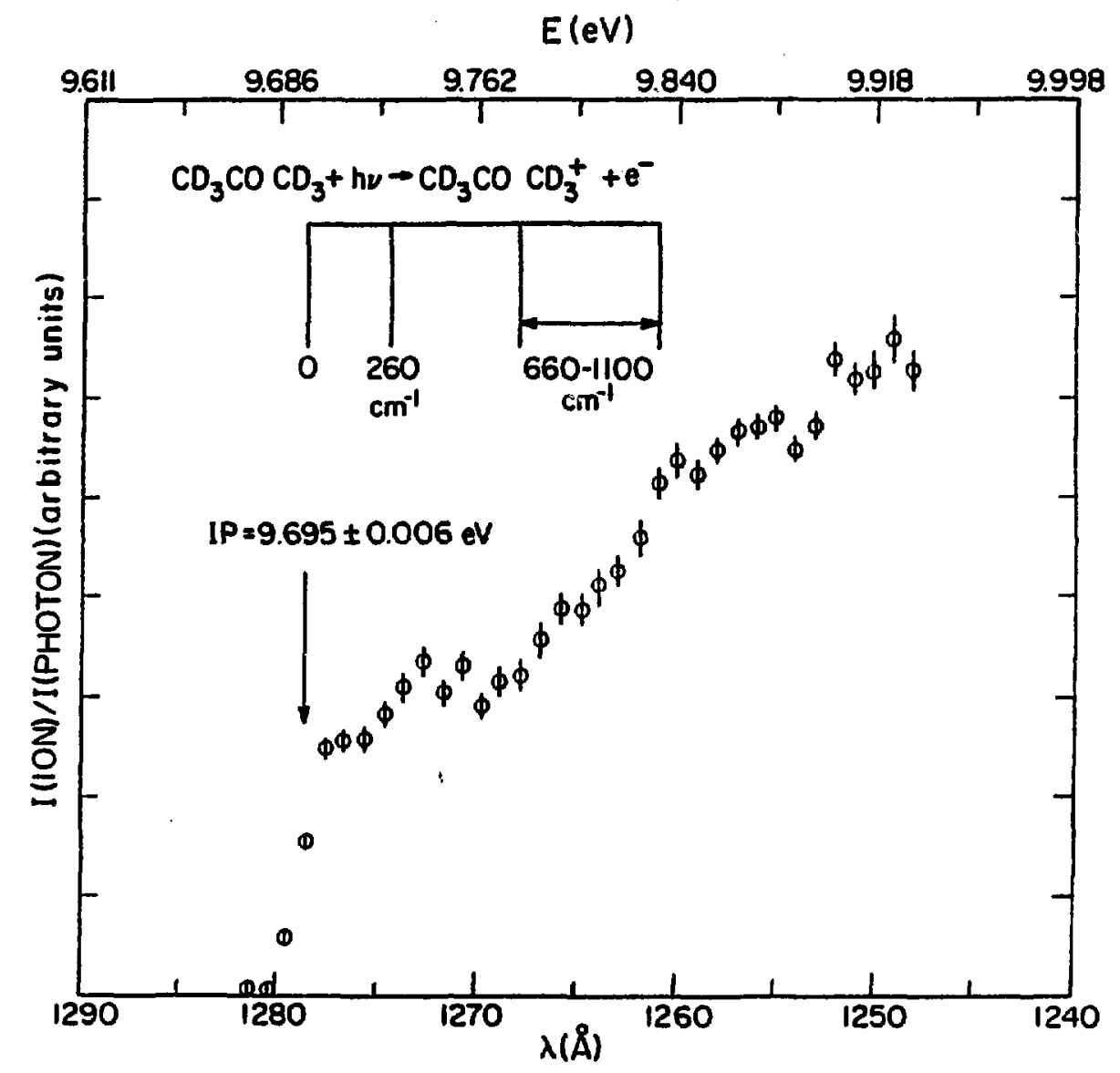

Figure 14.

Photolon Yield Curve for $\left(\mathrm{CD}_{3} \mathrm{COCD}_{3}\right)^{+}$ from 1248 to $1282 \AA$. 


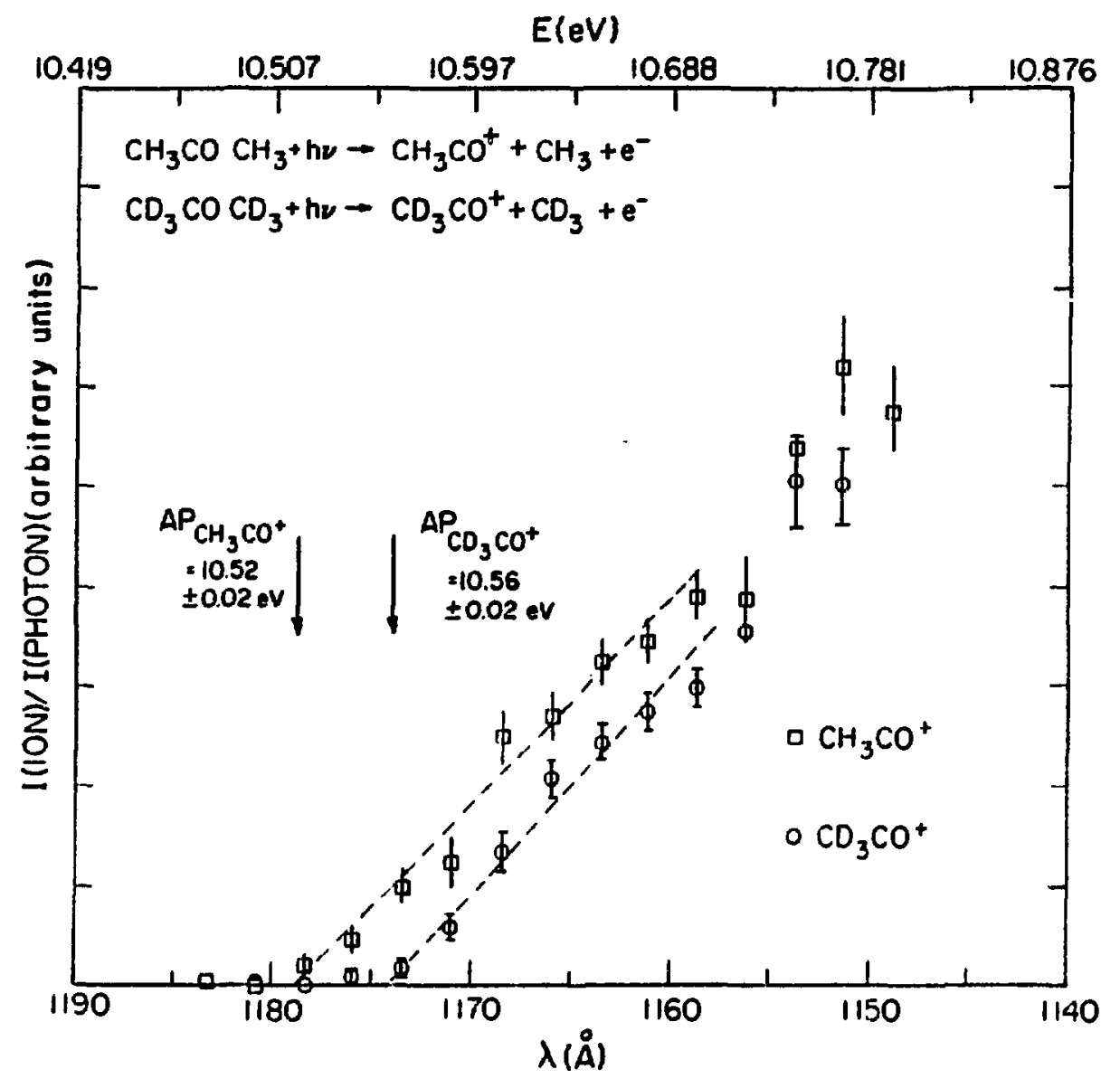

Figure 15. Appearance Potential Curves for $\mathrm{CH}_{3} \mathrm{CO}^{+}$
and $\mathrm{CD}_{3} \mathrm{CO}^{+}$from 1148 to $1183 \AA$. 


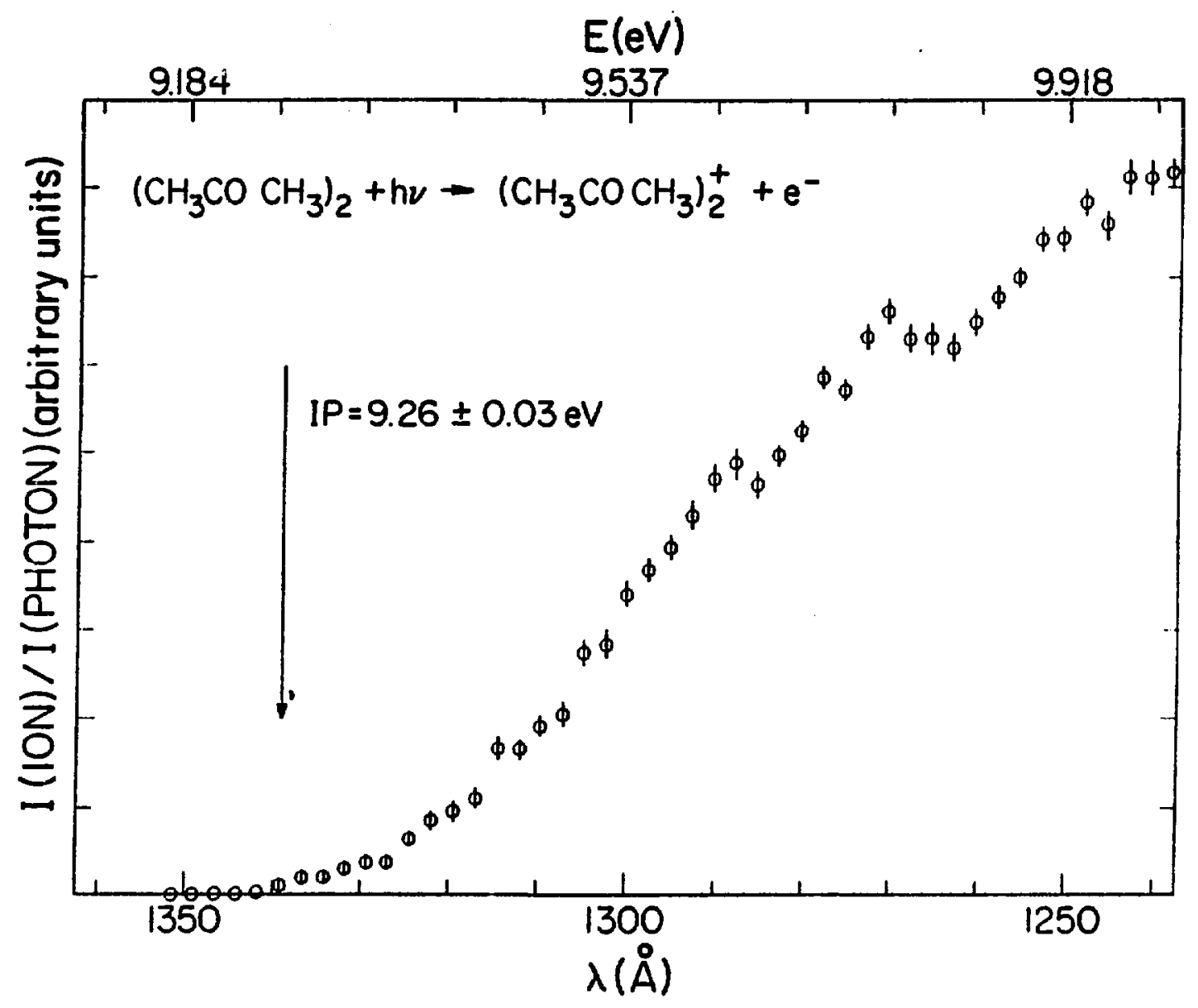

F1gure 16. Photolon Yield Curve for $\left(\mathrm{CH}_{3} \mathrm{COCH}_{3}\right)_{2}^{+}$ from 1239 to $1351 \AA$. 
$E(e V)$

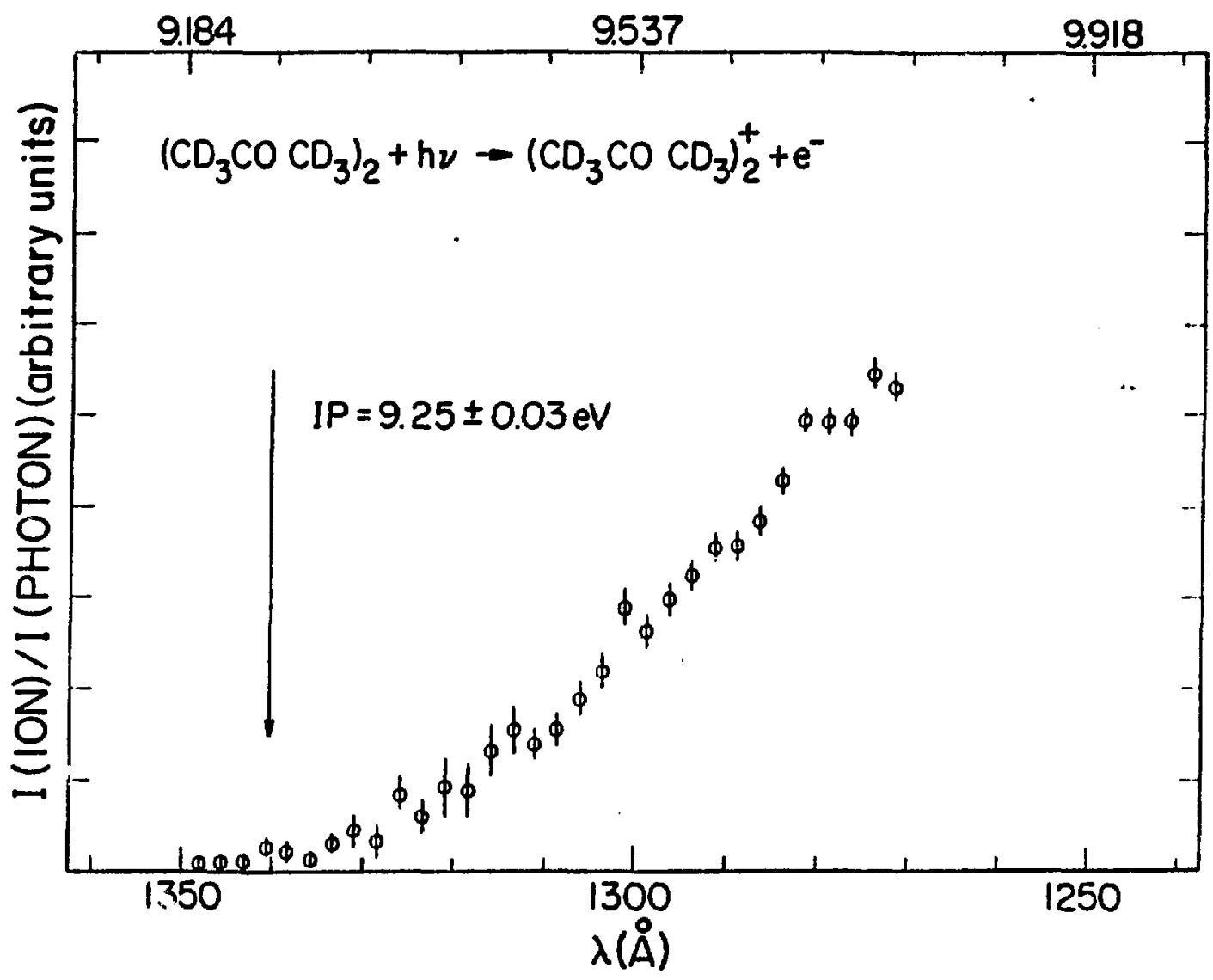

Figure 17.

Photoion Yleld Curve for $\left(C D_{3} C O C D_{3}\right)_{2}{ }^{+}$

from 1270 to 1349 \&. 


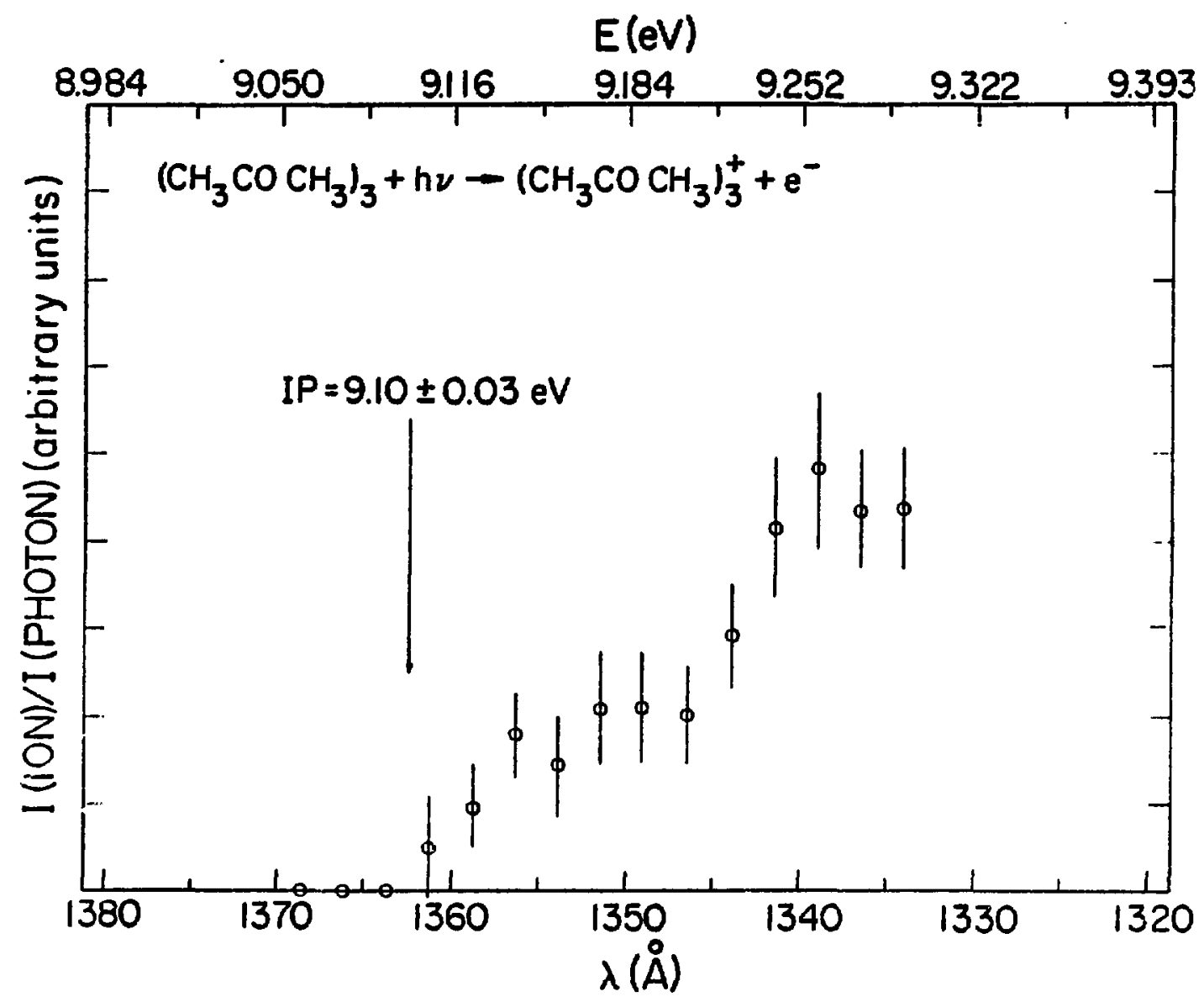

Figure 18.

$$
\begin{aligned}
& \text { Photoion yeld Curve for }\left(\mathrm{CH}_{3} \mathrm{COCH}_{3}\right)^{+} \\
& \text {from } 1334 \text { to } 1369 \AA .
\end{aligned}
$$




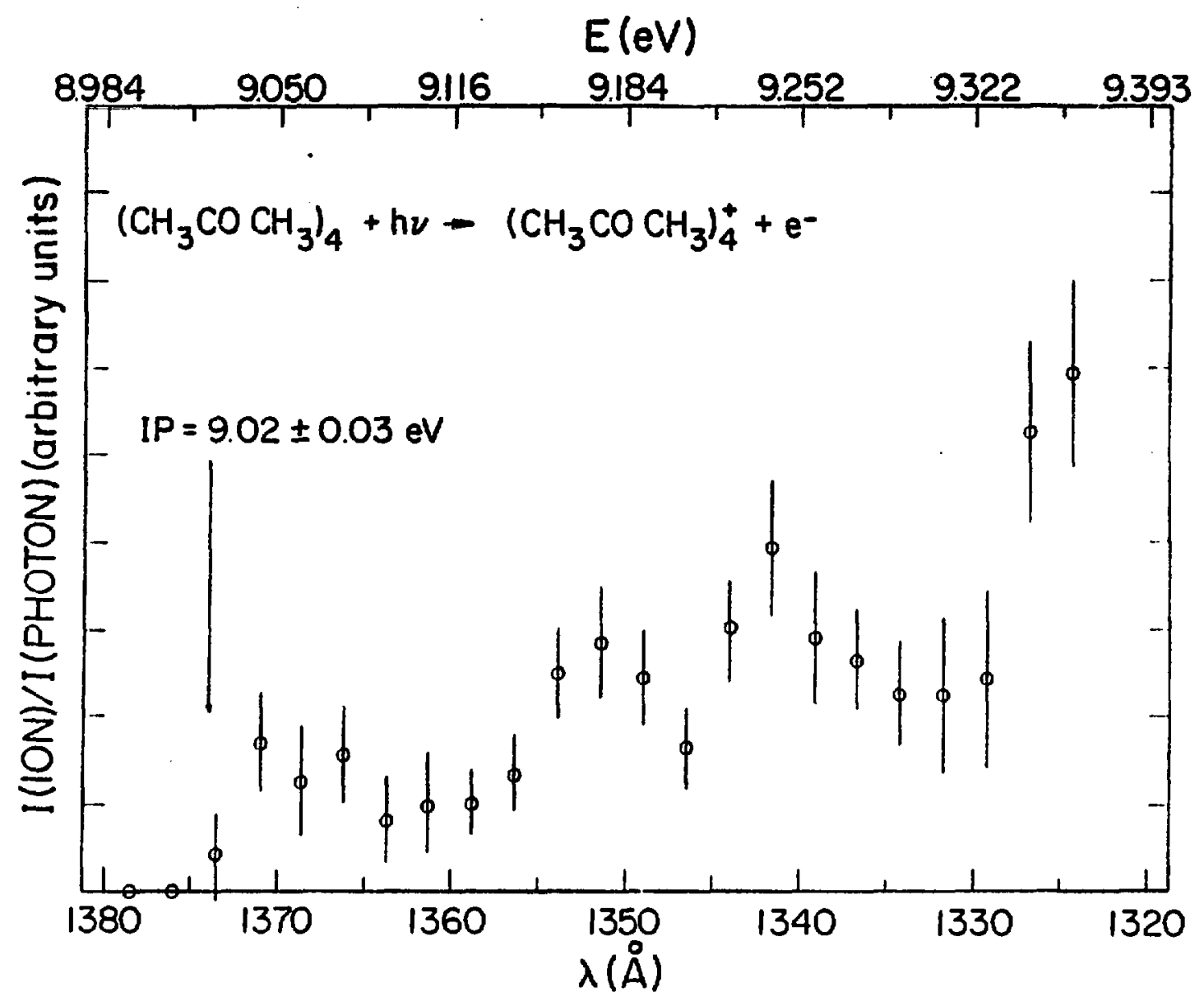

Figure 19. Photoion Yield Curve for $\left(\mathrm{CH}_{3} \mathrm{COCH}_{3}\right)_{4}+$ from 1324 to $1379 \AA$. 


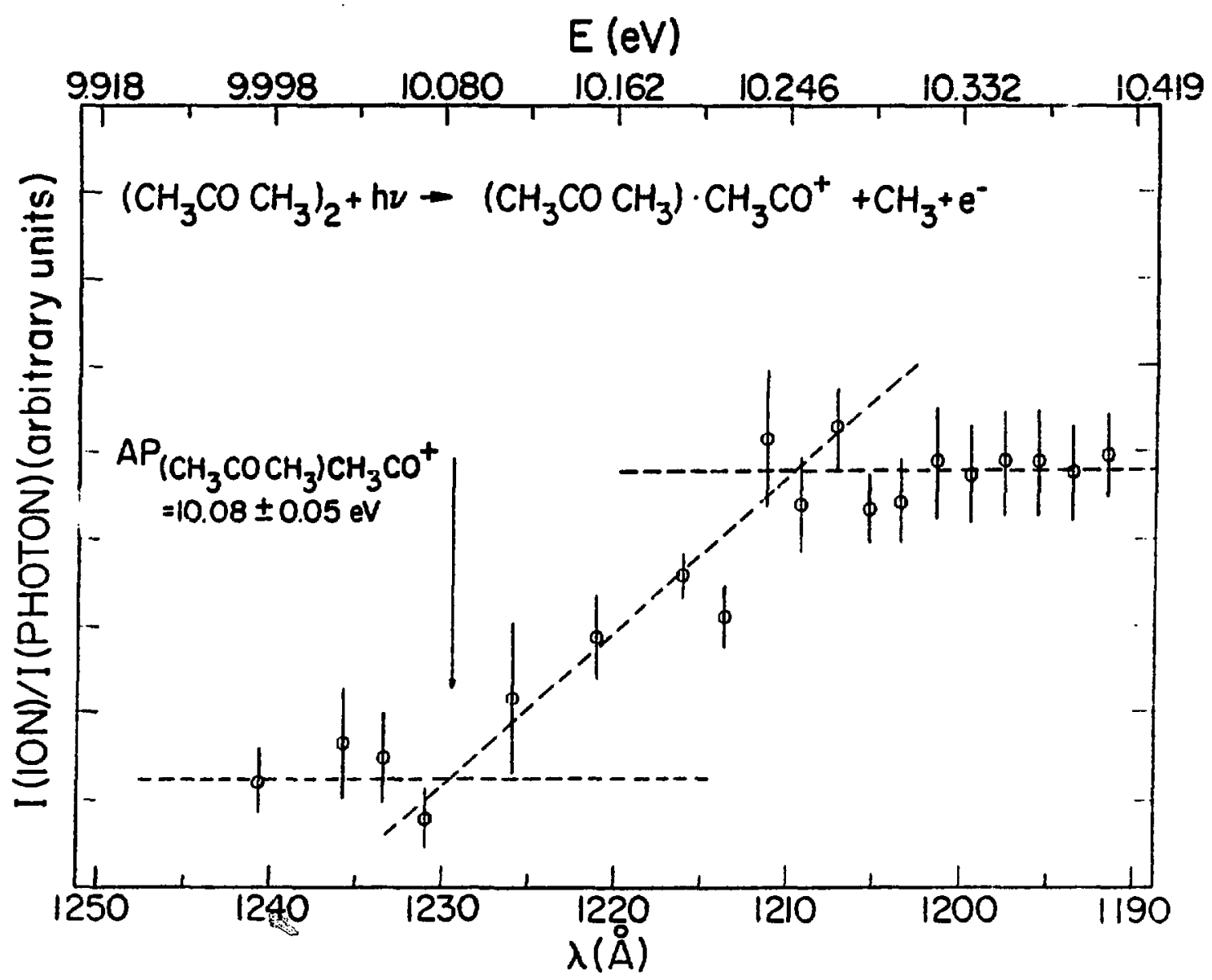

Figure 20.

Appearance Potential Curve for

$\left(\mathrm{CH}_{3} \mathrm{COCH}_{3}\right) \cdot \mathrm{CH}_{3} \mathrm{CO}^{+}$from 1190 to

$1241 \AA$. 
Table III. Summary of Energetic Information for

Acetone and Aceione- $\mathrm{d}_{6}$ Parent,

Fragment and Cluster Ions

Ion1zation or Appearance Potential (eV)

\begin{tabular}{|c|c|c|c|c|c|}
\hline Ion & m/e & Ihls Work & PI literature ${ }^{a}$ & PES Literature & $\begin{array}{c}\text { Ionic } \\
\text { vibrational } \\
\text { structure } \\
\end{array}$ \\
\hline $\mathrm{CH}_{3} \mathrm{COCH}_{3}^{+}$ & 58 & $9.694 \pm 0.006$ & $\begin{array}{l}9.71 \pm 0.01^{237} \\
9.69 \pm 0.01^{45}\end{array}$ & $\begin{array}{l}9.709 \pm 0.005^{258} \\
9.70 \pm 0.01^{261} \\
9.72 \pm 0.01^{266}\end{array}$ & $\begin{array}{l}320 \pm 50 \mathrm{~cm}^{-1} \\
695 \pm 50 \mathrm{~cm}^{-1} \\
930-1370 \mathrm{~cm}^{-1}\end{array}$ \\
\hline $\mathrm{CD}_{3} \mathrm{COCD}_{3}^{+}$ & 64 & $9.695 \pm 0.006$ & & & $\begin{array}{l}260 \pm 50 \mathrm{~cm}^{-1} \\
660-1100 \mathrm{~cm}^{-1}\end{array}$ \\
\hline
\end{tabular}

$\begin{array}{lll}\mathrm{CH}_{3} \mathrm{CO}^{+} & 43 & 10.52 \pm 0.02 \\ & & \\ & & \\ \mathrm{CD}_{3} \mathrm{CO}^{+} & 46 & 10.56 \pm 0.02 \\ \left(\mathrm{CH}_{3} \mathrm{COCH}_{3}\right)_{2}^{+} & 116 \cdot & 9.26 \pm 0.03 \\ \left(\mathrm{CD}_{3} \mathrm{COCD}_{3}\right)_{2}^{+} & 128 & 9.25 \pm 0.03 \\ \left(\mathrm{CH}_{3} \mathrm{COCH}_{3}\right)_{3}^{+} & 174 & 9.10 \pm 0.03 \\ \left.\left(\mathrm{CH}_{3} \mathrm{COCH}\right)_{3}\right)_{4}^{+} & 232 & 9.02 \pm 0.03 \\ \left(\mathrm{CHI}_{3} \mathrm{COCH}_{3}\right) \cdot \mathrm{ClI}_{3} \mathrm{CO}^{+} & 101 & 10.08 \pm 0.05\end{array}$

a. Selected photolonsation literature values.

b. Selected photoelectron sptecroscopy literature values.

c. 'Wls value determired by addlur, the calculated internal energy of acetone at room tenperature

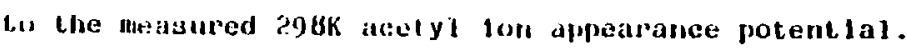


by sharp thresholds and distinct steplike structure at higher energles. The well-resolved fine structure and the apparent absence of hot band effects are ilrect results of the efflclent thermal relaxation achleved by sampling from a supersonic nozzle expansion. With1n the estimated resolution of $1.5 \AA$, the observed first 1onization onsets are very nearly step functions. This result wculd indicate that the rotational envelope for acetone has been narrowed to approximately a width of $12 \mathrm{meV}$ or less through supersonic gas expansion; i.e., the rotational temperature of the sample is $\approx 100 \mathrm{~K}$. Rotational temperatures of $\leq 118 \mathrm{~K}$ have been achieved in previous nozzle beam photolonfzation studies.79,90 Similarly, vibrational temperatures are apparently lowered to the extent that significant population of states occurs only within a narrow energy band above the ground state. The measured ionization potential of $\mathrm{CH}_{3} \mathrm{COCH}_{3}$ is in good agreement with previous photoionization and photoelectron spectroscopy results (see Table III). Ionization probably occurs at a $2 p$ lone-pair electron site on the oxygen atom, 286 resulting in a large $v^{\prime=}=0$ to $v^{\prime}=0$ onset. 36 In addition, the observed photolon yleld curve exhiblts several fine structure features within $0.2 \mathrm{eV}$ of threshold, including two abrupt steps at $320 \mathrm{~cm}^{-1}$ and $595 \mathrm{~cm}^{-i}$ and a broad rise from 
about $930-1370 \mathrm{~cm}^{-1}$. The estimated uncertainty of these values is $\pm 50 \mathrm{~cm}^{-1}$. Inasmuch as identical structure was not seen at the appropriate energles in a crude photoIonization efficiency curve determination of diethyl ether (IP $=9.55-9.60 \mathrm{eV}$ ), It may be concluded that these steps are not experimental artifacts arising from stray light or the type of photon source used. Rather, these features presumably occur because sufficlent excess energy is avaliable to excite higher vibrational levels of the 1on. Removal of a nonbonding electron should have a minimal effect on many low energy vibrational frequencles of the molecule since these frequently correspond to structural deformations. Consequentiy, it should be possible to correlate the observed lonic transitions with known low energy vibrations in acetone. 287,288

Steps corresponding to measured methyl group torsional frequencies in neutral acetone $v_{12}=$ $105.3 \mathrm{~cm}^{-1}$ and $\nu_{24}=108.4 \mathrm{~cm}^{-1}$ are not seen $1 \mathrm{n}$ this work. Since the ionic torsional modes are not totaly symmetric (assuming $\mathrm{CH}_{3} \mathrm{COCH}_{3}$ retains $\mathrm{C}_{2 \mathrm{v}}$ symmetry uoon ionization), vibrational selection rules indicate that transitions to these levels from the neutral acetone ground state are symmetry forbidden. 26,93 Also, it is quite likely that the target molecules do not experlence sufficient ribratianal relaxation duntrg the expansion 
to permit observation of these very low energy transitions. Indeed, these frequencles lie nearly at the Iimit of the experimental optical resolution. The onset of $320 \mathrm{~cm}^{-1}$ appears to correspond with the $A_{1}$ symmetric C-C-C deformation mode $\left(v_{8}\right)$, to which is assigned the $385 \mathrm{~cm}^{-1}$ band in the vibrational spectra of the neutral molecule. This ionic transition was tentatively 1dentifled in a previous photoelectron spectroscopy study. 256 Several normal modes could conceivably give rise to the observed feature at $695 \mathrm{~cm}^{-1}$, including the symetric C-C stretch $\left(v_{7}\right)$ and two out-of-plane skeletal fundamentals $\left(v_{19}, v_{23}\right)$. In neutral acetone, these modes occur at $777 \mathrm{~cm}^{-1}, 530 \mathrm{~cm}^{-1}$ and $484 \mathrm{~cm}^{-1}$, respectively. Since the out-of-plane vibrations involve the carbonyl group directly, these frequencies could be substantially altered upon ionization. To a lesser extent, a shift is expected for the $\mathrm{C}-\mathrm{C}$ stretch as well. Within the estimated resolution limits, a possible contribution from $2 v_{8}$ must also be considered. A specific assignment for this transition is not attempted; however, the vibrational selection rules favor the $A_{1}$ symmetric modes $\left(\nu_{7}, 2 \nu_{8}\right)$. In previous studies, an ionic vibrational feature has been observed at $1200 \mathrm{~cm}^{-1}$ which has been assigned to either the c-0 stretch 237 or the methyl group symmetric deformation mode.26E In the present work, this feature 
appears significantly broader than the lower energy transitions. It seems likely that the manifold at 930-1370 $\mathrm{cm}^{-1}$ arises from several unresolved vibrational excltations, possibly including the $\mathrm{CH}_{3}$ asymmetric and symmetric deformation modes $\left(v_{4}, v_{5}, v_{15}, v_{16}, v_{21}\right)$, the $\mathrm{CH}_{3}$ rocking modes $\left(v_{6}, v_{11}, v_{18}, v_{22}\right)$ and the asymmetric $C-C$ stretch $\left(\nu_{17}\right)$. A broad peak centered at about $1300 \mathrm{~cm}^{-1}$ in the acetone electron energy loss spectrum has been interpreted in a similar fashion. 277 Again, the $A_{1}$ symmetric modes $\left(\nu_{4}, \nu_{5}, v_{6}\right)$ are savored by the selection rules. At energies above $1400 \mathrm{~cm}^{-1}$ from threshold, the photoion yleld curve becomes very complex. This is due principaliy to the increasing superposition of ionization continua and the onset of dissociative processes. The occurrence of autoionization at $0.1-0.2 \mathrm{eV}$ above threshold has also been suggested. 259 Within stated error limits, the measured ionization potential of $\mathrm{CD}_{3} \mathrm{COCD}_{3}$ is identical to that for the acetone parent (see Table III). FIne structure in the acetone-d6 photolonization curve consists of a transition at $260 \mathrm{~cm}^{-1}$ and a broad rise from about $660-1100 \mathrm{~cm}^{-1}$. In the vibrational spectra of the neutral molecule, perdeutero substitution has the effect of reducing $\nu_{3}$ by about $65 \mathrm{~cm}^{-1}$ and lowering the 
$\mathrm{CH}_{3}$ rocking and deformation modes by $160-400 \mathrm{~cm}^{-1} \cdot 288$ A reduction in $\nu_{7}$ and $\nu_{I 7}$ by about $100 \mathrm{~cm}^{-1}$ and $200 \mathrm{~cm}^{-1}$ respectively is also observed. These results favor the assignment of the $260 \mathrm{~cm}^{-1}$ mode to $v_{8}$ and the .1gher energy manifold to a composite of $C-C$ stretches $\left(v_{7}, v_{17}\right)$ and methyl group excitations. Vibrational selection rules apply as in the case of $\mathrm{CH}_{3} \mathrm{COCH}_{3}$.

In view of the aforementioned wavelength dependence of sodlum salicylate flourescence quantum efficiency between 1250-1500 $\AA$ (see Section III.B.), care must be exercised in interpreting the parent ion efficiency curves over a wide energy range; however, this effect is undoubtedly smali $(<0.5 \%)$ for individual features occurring in less than $30 \mathrm{meV}$. Consequently, it should be possible to derive falriy accurate relative Franck-Condon factors from the step helghts of the observed structure near threshold. For $\mathrm{CH}_{3} \mathrm{COCH}_{3}{ }^{+}$, the measured relative transition probabilities for the first three onsets are 1.00:0.35:0.12. The relative values for the first two steps in the $C_{3} \mathrm{COCD}_{3}{ }^{+}$ curve are about 1,00:0.25. It must be emphasized tiat the inftial thresholds may include some contribution by unresolved transitions to (and from) the low energy methyl group torsional modes. 


$$
\begin{aligned}
& \text { b. } \mathrm{CH}_{3} \mathrm{CO}^{+} \text {and } \mathrm{CD}_{3} \mathrm{CO}^{+} \text {fragment fors } \\
& \text { Appearance potential curves for }
\end{aligned}
$$

acetyl fragment fons from acetone and acetone- $d_{6}$ are shown in Figure 15. The measured threshold value of $10.52 \pm 0.02 \mathrm{eV}$ for $\mathrm{CH}_{3} \mathrm{CO}^{+}$is $0.1-0.2 \mathrm{eV}$ higher than previous room temperature determinations (see Table III) and is, in fact, $0.07 \mathrm{eV}$ higher than the calculated $\mathrm{OK}$ value of Murad and Inghram. 255 The relatively high appearance potentlal and the near absence of curvature at onset provide evidence of substantial thermal relaxation of the acetone parent molecules in the present work. In view of the apparent uncertainty in the earlier measurements, 237,255,256 the present determination strongly suggests that the $O K$ appearance potential is likely $10.54 \mathrm{eV}$ or higher.

Murad and Inghram have used their extrapolated OK acetyl ion appearance potential of $10.45 \mathrm{eV}$ to derive several thermodynamic quantities.255,256 In these derivations, the kinetic shift in the fragmentation of acetone to form $\mathrm{CH}_{3} \mathrm{CO}^{+}$was estimated to be $0.25 \mathrm{eV}$. Subsequent calculations based on quasi-equilibrium theory Indicate that this value is Iikely to be $0.01 \mathrm{eV}$ or less. 131 since the present study favors an appearance potent1al as much as $0.1 \mathrm{eV}$ higher than $10.45 \mathrm{eV}$, a 
cumulative error of up to $0.35 \mathrm{eV}$ for the deduced thermochemical information may have resulted from the low threshold value and overestimated kinetic shift. These thermodynamic quantities are not recalculated since there is no certain means of determining or estimating the OK appearance potential with the apparatus as constructed.

The observed threshold for the dissociation of acetone- $d_{6}$ to form $\mathrm{CD}_{3} \mathrm{CO}^{+}$is $10.56 \pm 0.02 \mathrm{eV}$. This represents a secondary isotope effect in the appearance potential of about $0.04 \mathrm{eV}$ upon perdeuteration. This effect was clearly observed in three separate measurements of the $\mathrm{CH}_{3} \mathrm{CO}^{+}$and $\mathrm{CD}_{3} \mathrm{CO}^{+}$onsets. By comparison, a primary isotope effect of $0.092 \mathrm{eV}$ has been seen in the formation of $\mathrm{HCO}^{+}$and $\mathrm{DCO}^{+}$from $\mathrm{H}_{2} \mathrm{CO}$ and $D_{2} \mathrm{CO}$ respectively. 138

\section{c. Acetone and acetone- $\mathrm{d}_{6}$ cluster fons} The photoionization efficiency curve for $\left(\mathrm{CH}_{3} \mathrm{COCH}_{3}\right)_{2}^{+}$is shown in Figure 16. In contrast to the sharp thresholds obtained in the monomer work, the photolon yield of the dimer exhibits a weak orset and generally increases slowly as a function of excess energy. This behavior has been observed in previous cluster studies $83,84,90$ and is best explained by the possibility of a substantial change in the poitential enersy surface 
upon 1onization. For example, Muliken estimates a $1.55 \&$ reduction in the equilibrium location of the potentlal well minimum for $\mathrm{Xe}_{2}+\mathrm{Xe}_{2}{ }^{+} .289$ Under these c1rcumstances, the Franck-Condon factor for direct Ionlzation may be quite small (see Figure 2). Indeed, the possibility exists that the adiabatic transition w11l not be seen above background. 83 Within the sensitivity of the present experiment, the ionization potential of $\left(\mathrm{CH}_{3} \mathrm{COCH}_{3}\right)_{2}$ is observed at $9.26 \pm 0.03 \mathrm{eV}$. Much of the fine structure in the dimer ion curve is reproducible; however, specific assignments are not attempted in view of the low resolution employed in this measurement, the obvious complexity in the vibrational structure of this system, and the unknown contribution. to the photolon yield above threshold due to fragmentation of higher clusters. 83 Because of the ratios of dimer ions to higher cluster ions observed in the present work (see Section IV.B.2.), the latter effect is expected to be small.

The Ionization potential determination for $\left(\mathrm{CH}_{3} \mathrm{COCH}_{3}\right)_{2}$ permits the calculation of the dimen ion binding energy by consideration of the following reactions:

$$
\mathrm{CH}_{3} \mathrm{COCH}_{3}+\mathrm{CH}_{3} \mathrm{COCH}_{3}-\mathrm{CH}_{3} \mathrm{COCH}_{3}^{+}+\mathrm{CH}_{3} \mathrm{COCH}_{3}+e^{-}
$$




$$
\begin{aligned}
\mathrm{CH}_{3} \mathrm{COCH}_{3}+\mathrm{CH}_{3} \mathrm{COCH}_{3} & \rightarrow\left(\mathrm{CH}_{3} \mathrm{COCH}_{3}\right)_{2} \\
\left(\mathrm{CH}_{3} \mathrm{COCH}_{3}\right)_{2} & +\left(\mathrm{CH}_{3} \mathrm{COCH}_{3}\right)_{2}^{+}+e^{-} \\
\left(\mathrm{CH}_{3} \mathrm{COCH}_{3}\right)_{2}^{+}+\mathrm{e}^{-} & +\mathrm{CH}_{3} \mathrm{COCH}_{3}^{+}+\mathrm{CH}_{3} \mathrm{COCH}_{3}+e^{-} .
\end{aligned}
$$

The sum of the enthalpies for (83), (84) and (85)

equals the observed monomer lonization potential of $9.694 \pm 0.006 \mathrm{eV}$. No experimental value for the neutral dimer binding energy has been reported. However, an estimate of this quantity may be mace using known second virial coefficient data. Braun and Lefdecker have calculated the water dimer binding energy 290 (BE) by assuming an fdeal two component (monomer and bound dimer) mixture of $\mathrm{H}_{2} \mathrm{O}$ vapor for $\mathrm{K} \mathrm{T}<<\mathrm{BE}$. Under similar assumptions, the energy of formation $\Delta u(T)$ for Reaction (83) at fixed values of $T$ and $P$ is given by

$$
\frac{d}{d\left(\frac{l}{T}\right)} \text { in }\left[b_{0}-B_{2}(T)\right]=\frac{\langle\Delta u(T)\rangle}{R}
$$

where $b_{0}$ is the so-called "excluded volume" of the gas, $B_{2}(T)$ are the experimental second $v i r i a l$ coefficlents and $\langle\Delta u(T)\rangle$ is averaged over ail internal states of the dimer. As $T$ nears $O K,\langle\Delta u(T)\rangle$ approaches the binding energy of the dimeric species. Hence, by plotting the avallable $\ln \left[b_{0}-B_{2}(T)\right]$ data for acetone as a function of $1 / T$, the binding energy may be estimated from the low temperature slope of Equation (86). This procedure has 
been applied to several reported sets of $\bar{E}_{2}(T)$ data ${ }^{292-295}$ as shown in Table IV. Sriefly, calculated $B E$ values in the range $1.6-2.4 \mathrm{kcal} / \mathrm{mole}$ have been obtalned whlle the data of Anderson et. al. ${ }^{296}$ indicate a substantially higher value. Taking the average of these determinations, a reasonable estimate for the acetone dimer binding energy is about $2.4 \mathrm{kcal} / \mathrm{mole}$ or $0.104 \mathrm{eV}$. Using this value, Reactions $(82)-(85)$ give a dimer lon binding energy of $0.538 \mathrm{ev}$ or $12.4 \mathrm{kcal} / \mathrm{mole}$. The uncertainty in this quantity is probably about $\pm 1 \mathrm{kcal} / \mathrm{mole}$. In view of the possiblifty that the dimer adiabatic Ionization potential is not cbserved. $12.4 \mathrm{kcal} / \mathrm{mole}$ must be considered a lower bound. The photolon yield curve for $\left(\mathrm{CD}_{3} \mathrm{COCD}_{3}\right)_{2}^{+}$ is shown in Figure 17 . Within the resolution of the present work, the measured lonization potential of the acetone- $d_{6}$ dimer is identical to that of $\left(\mathrm{CH}_{3} \mathrm{COCH}_{3}\right)_{2}$. The $\left(\mathrm{CH}_{3} \mathrm{COCH}_{3}\right)_{2}{ }^{+}$and $\left(\mathrm{CD}_{3} \mathrm{COCD}_{3}\right)_{2}{ }^{+}$curves seem to exhibit considerable similarity in their fine structure, suggesting that the observed transitions are falrly insensitive to isotopic substitution. For reasons previousiy stated, specific assignments are not attempted.

Flgures 18 and 19 contain the photolonization efficiency curves near threshold for $\left(\mathrm{CH}_{3} \mathrm{COCH}_{3}\right)_{3}{ }^{+}$ and $\left(\mathrm{CH}_{3} \mathrm{COCH}_{3}\right)_{4}{ }^{+}$. Ionization potentials for 
Table IT. Salculated Acetore Dimer Esnding

A. Reference 292 .

$$
\text { Energles from } \Xi_{2} \text { (T) Data. }
$$

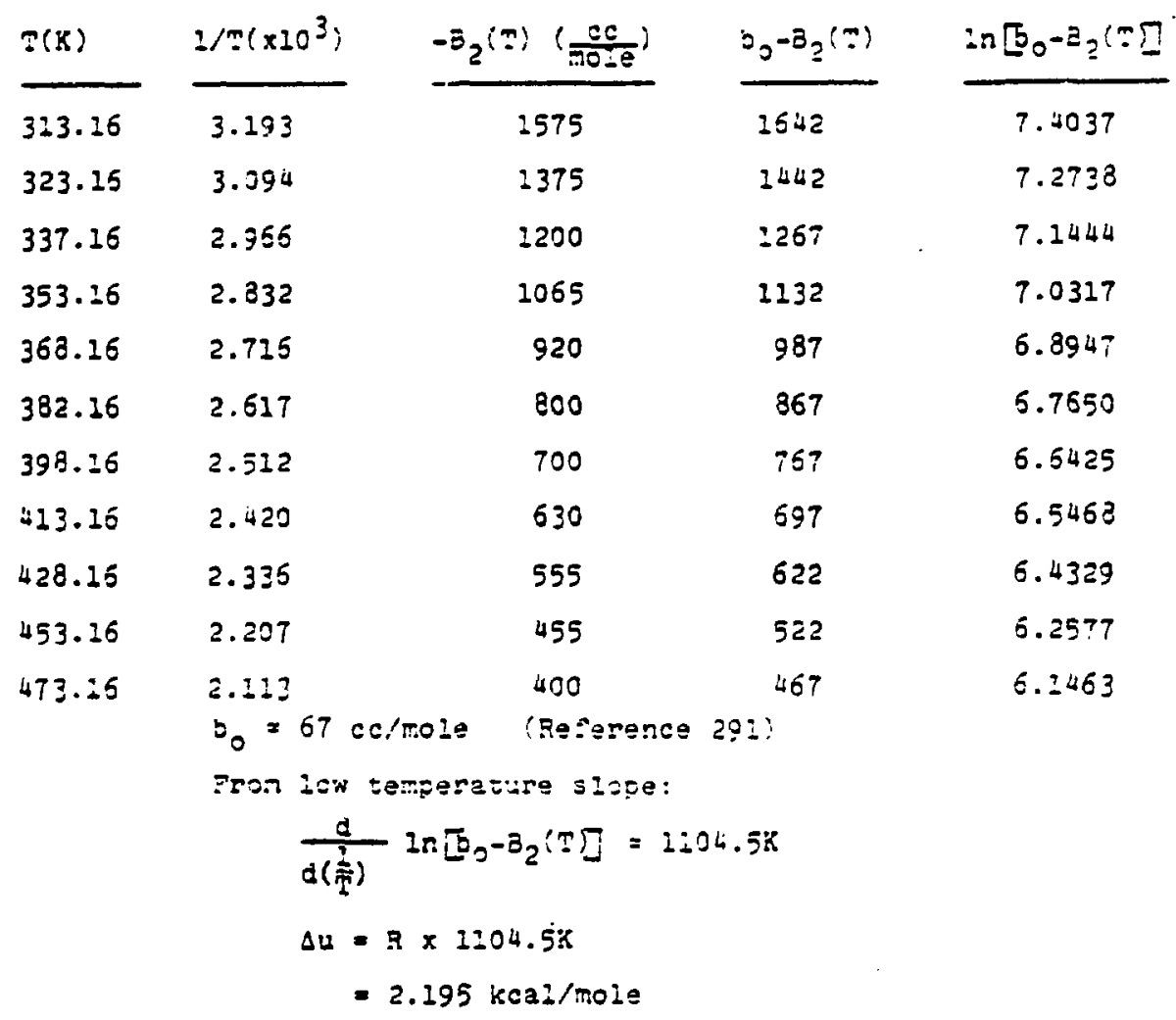

3. Reference 293 .

\begin{tabular}{|c|c|c|c|c|}
\hline$=(x)$ & $1 /=\left(x \geq 0^{3}\right)$ & $-B_{2}(\pi)\left(\frac{n}{\pi+3}\right)$ & $b_{0}-B_{2}(z)$ & $\therefore\left[E_{0}-a_{2} ;=\square\right.$ \\
\hline 300 & 3.333 & $2000 \pm 200$ & $2067 \pm 220$ & $7.5321-7.5339-7.72 \leq 2$ \\
\hline 310 & 3.226 & $1730 \pm 100$ & $1797 \pm 102$ & $7 .+366-7.4939-7.3050$ \\
\hline 320 & 3.125 & $1520 \pm 50$ & $2587 \pm 50$ & $7.3376-7.35: E-7 .+E 0 z$ \\
\hline 330 & 3.030 & $1350 \pm 50$ & $1417 \pm 50$ & $7.2204-7.3563-7.2922$ \\
\hline 340 & 2.941 & $2200 \pm 50$ & $2257 \pm 50$ & $7.2041-7.2444-7.2832$ \\
\hline 350 & 2.357 & $=080 \pm 50$ & $2: 47 \pm 50$ & $7.0003-7.0453-7.23-2$ \\
\hline 360 & $\begin{array}{l}2.779 \\
b_{0}=67 \mathrm{cc} /\end{array}$ & $\begin{array}{l}960 \pm j 0 \\
\text { e. (Reference }\end{array}$ & $\begin{array}{l}2027 \pm 50 \\
\text { 1) }\end{array}$ & $5.334 E-\varepsilon .9345-6.98 i 9$ \\
\hline
\end{tabular}$$
\frac{d}{d\left(\frac{T}{T}\right)} \ln \left[5_{0}-5_{2}(T)=2215.3 \mathrm{~K}\right.
$$$$
\Delta u=2.425 \mathrm{kcal} / \mathrm{mele}
$$ 
S. Reference 234 .

\begin{tabular}{|c|c|c|c|c|}
\hline$T(K)$ & $\left.2 / 2 ! \times 10^{3}\right)$ & $-E_{2}(T)\left(\frac{a}{x-5}\right)$ & $b_{g}-\equiv_{a}(m)$ & $\therefore=\bar{z}_{0}-\bar{a}_{2}(-]$ \\
\hline 291.7 & $3 .+29$ & 2075.9 & 2142.9 & 7.5699 \\
\hline 319.4 & 3.131 & 2544.1 & 1711.1 & 7.4449 \\
\hline 329.5 & 3.035 & 1527.3 & 2594.3 & 7.3742 \\
\hline 335.0 & 2.985 & 1406.3 & 1473.3 & $i .2953$ \\
\hline 341.4 & 2.929 & 1342.2 & 1409.2 & 7.2508 \\
\hline 351.2 & 2.847 & 1257.5 & 1324.5 & 7.2388 \\
\hline 361.2 & 2.769 & 1133.6 & 1200.6 & 7.0905 \\
\hline 368.4 & 2.714 & 965.4 & 1032.4 & 6.9396 \\
\hline 385.0 & 2.597 & 353.9 & 920.9 & 6.3254 \\
\hline 405.6 & $\begin{array}{l}2.465 \\
b_{0}=67\end{array}$ & $\begin{array}{l}650.5 \\
\text { (Reserenc }\end{array}$ & $232)^{717.6}$ & 6.5759 \\
\hline
\end{tabular}

From low temperature slope:

$$
\begin{aligned}
& \frac{d}{d\left(\frac{1}{2}\right)} \ln \left[5_{0}-a_{2}(2]=323.2 \mathrm{~K}\right. \\
& \Delta u=1.626 \mathrm{kcal} / \mathrm{mole}
\end{aligned}
$$

\begin{tabular}{|c|c|c|c|c|}
\hline$T(K)$ & $1 / T\left(\times 10^{3}\right)$ & $-a_{2}(T)\left(\frac{c c}{d \sigma-a}\right)$ & $b_{0}-\bar{s}_{2}(\pi)$ & $\ln \left[\sigma_{0}-a_{2}(\pi]\right.$ \\
\hline 323.16 & 3.094 & 1560 & 1627 & 7.3945 \\
\hline 343.16 & 2.314 & 1280 & 1347 & 7.2056 \\
\hline 363.15 & 2.754 & 1040 & 2107 & 7.0094 \\
\hline 383.16 & 2.620 & 850 & 927 & $6.381:$ \\
\hline 403.16 & $\begin{array}{l}2.420 \\
b_{0}=67\end{array}$ & $\begin{array}{l}700 \\
\text { (:eferene }\end{array}$ & $91)^{757}$ & $6.6+25$ \\
\hline
\end{tabular}

D. Reference 295.

From Low temperasure slope:

$$
\begin{aligned}
& \frac{d}{d\left(\frac{1}{T}\right)} \ln \left[5_{0}-z_{2}(5)\right]=1298.2 \mathrm{x} \\
& \Delta u=2.381 \mathrm{kcal} / \mathrm{role}
\end{aligned}
$$

E. Reference 296 .

$$
\Delta u=\Delta H_{2}=-3.9 \mathrm{kcal} / \mathrm{mole}
$$


these species are observed at $9.10 \pm 0.03 \mathrm{eV}$ and $9.02 \pm 0.03 \mathrm{eV}$ respectively. Again, it is not certain that these values represent the adiabatic transitions. The extremely low count rates $(0.03-0.1$ count/sec) In these measurements prevent any useful analysis of the fine structure.

With positive ion energetic information avaliable for polymers up to the tetramer, it becomes possible to investigate the relationship between cluster slze and bulk properties. For example, it has been observed that the lonization potential for an alkall metal aggregate contalning as few as three or four atoms approaches the calculated effective work iunction of a continuous metal drop of the same size.61,297,298 In contrast, the 1onization potentials for $(\mathrm{Hg})_{\mathrm{n}}$ do not fall rapidly toward the work function with increasing $n .299$ For compounds such as acetone, results of this type are likely to be of signiflcant interest in that they have a direct bearing on nucleation and condensation phenomena and possibly even on liquid structure.

In the present study, a smooth and systematic decrease in ionization potential with increasing acetone cluster size is observed. As indicated in Figure 21, the measured 1onization energles for $\left(\mathrm{CH}_{3} \mathrm{COCH}_{3}\right)_{\mathrm{n}}$ fall squarely on a straight line when plotted as a function of $1 / n$. It is interesting to compare this functional 


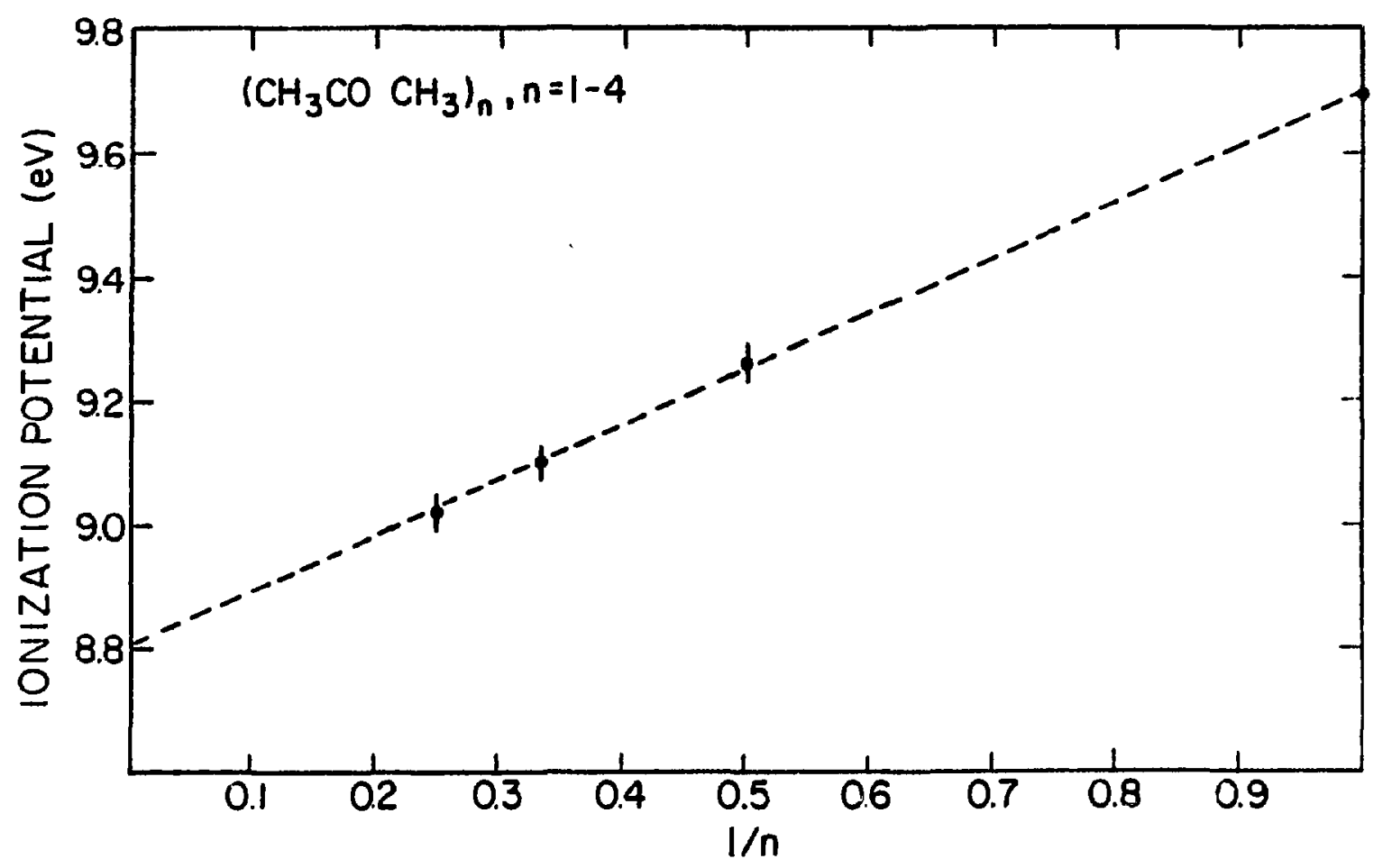

F1gure 21. Plot of the Observed Ionization

Energies for $\left(\mathrm{CH}_{3} \mathrm{COCH}_{3}\right)_{n}, \mathrm{n}=1-4$,

as a Function of $1 / n$. 
dependence to that which would be expected on the basis of a simple independent systems model 300 for the cluster Ion system. Using a straightforward perturbation approach, the energy levels for the indlvidual monomerlc units of a linear cluster ion are considered to undergo splitting due to weak nearest neighbor interactions. Long-range interactions are set equal to zero. The much smaller splittings due to the relatively weak Interactions in the neutral cluster are ignored. Under these assumptions, the secular equation describing the degenerate manifold for cluster ion size $n$ should be as follows :

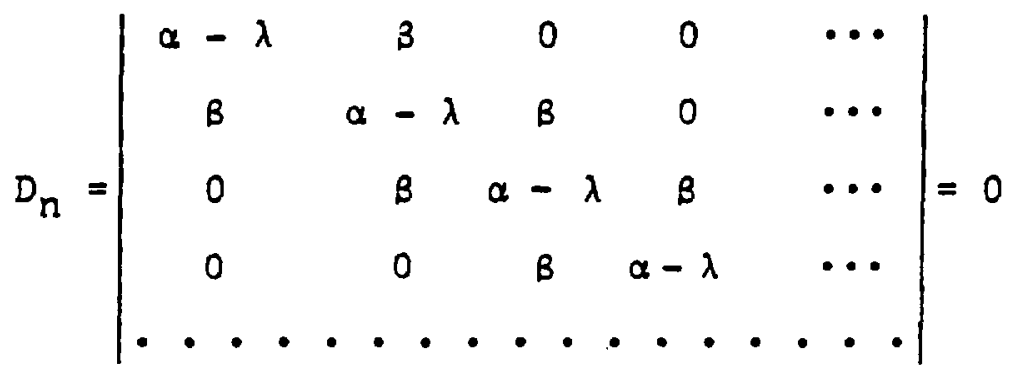

where $\alpha$ represents the monomer ionization potential and $\beta$ Is the nearest ne1ghbor 1nteraction parameter. It can be shown that the minimum transition energy $\lambda=\alpha-2 B \cos \left(\frac{\pi}{n+I}\right)$. To the extent that the above model accurately represents a cluster ion system, this implies a linear decrease of the ionization potential as a function of $\cos \left(\frac{\pi}{n+1}\right)$. Sinse cos $\left(\frac{\pi}{n+1}\right)$ is very nearly a linear function of $I / n$ for $n=2-10$, this model produces a.good correlation to our data for the first few members of 
$\left(\mathrm{CH}_{3} \mathrm{COCH}_{3}\right)_{n}$. Of course, this analysis does not establish a specific structure for the acetone cluster ions; however, it is intriguing that a satisfactory correlation of Ionization potential with cluster size can be achieved using this simple picture.

If the ionization energies for $\left(\mathrm{CH}_{3} \mathrm{COCH}_{3}\right)_{n}$, $\mathrm{n}>4$, continue to decrease IInearly as a function of $1 / n$, Flgure 21 predicts a bulk fonlzation potential of about $8.8 \mathrm{ev}$. A value of $8.86 \mathrm{eV}$ is calculated from a plot of ionization potential vs cos $\left(\frac{\pi}{n+1}\right)$. This sugsests that the Ionization energy of acetone is reduced by about $0.85 \mathrm{eT}$ upon condensation. In view of the uncertainty as to whether our measured cluster fon thresholds correspond to adiabatic transitions, $0.85 \mathrm{eV}$ is probably a lower bound. This value compares favorably to relaxation shifts of $1.0-1.65 \mathrm{eV}$ recently observed in the ultraviolet photoemission spectra of seven gases condensed on a $\mathrm{MoS}_{2}$ substrate. 301,302

The photolonization efficiency curve for the final ion investigated in acetone molecular beams, $\left(\mathrm{CH}_{3} \mathrm{COCH}_{3}\right) \cdot \mathrm{CH}_{3} \mathrm{CO}^{+}$, is shown in Figure 20 . Despite the very low count rate for this specle $(0.03-0.3$ count/sec), a definite onset is seen at $10.08 \pm 0.05 \mathrm{eV}$. This is taken to be the appearance potentlal for the process

$$
\left(\mathrm{CH}_{3} \mathrm{CCCH}_{3}\right)_{2} \rightarrow\left(\mathrm{CH}_{3} \mathrm{COCH}_{3}\right) \cdot \mathrm{CH}_{3} \mathrm{CO}^{+}+\mathrm{CH}_{3}+\mathrm{e}^{-} \text {. }
$$


The origin of the very few counts observed between $1230-1240 \AA$ is not certain. The desolvation energy, $\Delta \mathrm{H}_{\mathrm{S}}$, of $\left(\mathrm{CH}_{3} \mathrm{COCH}_{3}\right) \cdot \mathrm{CH}_{3} \mathrm{CO}^{+}$can be estimated from the enthalpies of Reactions (83), (38) and the following:

$$
\begin{gathered}
\mathrm{CH}_{3} \mathrm{COCH}_{3}+\mathrm{CH}_{3} \mathrm{COCH}_{3}+\mathrm{CH}_{3} \mathrm{COCH}_{3}+\mathrm{CH}_{3} \mathrm{CO}^{+}+\mathrm{CH}_{3}+e^{-} \\
\left(\mathrm{CH}_{3} \mathrm{COCH}_{3}\right) \cdot \mathrm{CH}_{3} \mathrm{CO}^{+}+\mathrm{CH}_{3}+\mathrm{e}^{-}+\mathrm{CH}_{3} \mathrm{COCH}_{3}+\mathrm{CH}_{3} \mathrm{CO}^{+} \\
+\mathrm{CH}_{3}+e^{-} .
\end{gathered}
$$

Using the experimental appearance potentials for (88) and (89) and $-0.104 \mathrm{eV}$ for the enthaipy of (83) as before, $\Delta \mathrm{H}_{\mathrm{S}}$ is calculated to be $0.544 \mathrm{eV}$ or $12.5 \mathrm{kcal} / \mathrm{mole}$. The similarlty between this value and the dimer ion binding energy calculated from Reactions (82) - (85) would seem to suggest that the methyl groups in acetone play a minimal role in the bonding of cluster lons.

\section{Preliminary Photoionization Experiments on Carbon Disulfide}

As a simple triatomic inorganic molecule, $\mathrm{CS}_{2}$ may be expected to exhibit slgnificantly different ionization and fragmentation proparties than those seen in complex organic compounds sucin as acetone. These properties, especially the first and higher ionization. potentials, have in fact been widely studied by various techniques including vacuum ultraviolet absorption spectroscopy, 303, 324 chototonization, $45,122,305$ photoelectron spectroscopy $124,306-310$ and photoionization. 
resonance spectroscopy (threshold photoelectron spectroscopy). 123 Because of the intense autolonization features which dominate the photolon yield curve of the parent $\mathrm{CS}_{2}{ }^{+}$Ion (see Section II.B.2.a.), photoelectron spectroscopic methods are far superior for the investigation of direct fonization processes occurring well above the inftial threshold; however, photoionization mass spectrometry may be profitably applied to the measurement of transitions near the first ionization limlt and also appearance potentials for dissociative processes. In particular, it would be worthwhile to use high resolution molecular beam photoionization methods to determine the excitation energies for $\mathrm{CS}_{2}{ }^{+}$ Ionic vibrational modes and to compare these energies with avallabie values obtained from other techniques. 123,308,311 Moreover, the photoionization efficiency curve of $\left(\mathrm{CS}_{2}\right)_{2}{ }^{+}$ is of interest inasmuch as an analogous experiment has been performed on $\left(\mathrm{CO}_{2}\right)_{2}{ }^{+} \cdot 83,91$ The present study reports a preliminary low optical resolution measurement of the $\mathrm{CS}_{2}{ }^{+}$photo1on $\mathrm{y}$ ield curve between $1200-1240 \AA$ as well as a low resolution determination of the $\left(\mathrm{CS}_{2}\right)_{2}{ }^{+}$curve in the wavelength range 1000-1290 $\AA$.

\section{Experimental}

Molecular beam sampling of carbon disulfide was accomplished oy atzaching directry to the sas manifoid 
a flask containing liquid $\mathrm{CS}_{2}$ at $20-25^{\circ} \mathrm{C}$, thereby sustaining a nozzle stagnation pressure of at least 365 torr. For the dimer study, the $\mathrm{CS}_{2}$ vapor was seeded in enough $\mathrm{H}_{2}$ to bring the total source pressure to about 700 torr. A f1xed $\mathrm{CS}_{2}$ vapor pressure was maintained by immersing the Ilquid sample container in a constant temperature $\left( \pm 1^{\circ} \mathrm{C}\right)$ bath. Natheson, Coleman and Bell $\mathrm{CS}_{2}$ reagent, A.C.S., was used without further purification.

An optical resolution of about $3.75 \AA$ (or $30 \mathrm{meV}$ ) was employed for both the monomer and dimer photolon yield curves $(500 \mu$ monochromator entrance and exit slits). Operating conditions for the hydrogen lamp were very similar to those used in the acetone work; however, the avallable nonochromatic light intensities were significantly below those obtalned in the early $\mathrm{CH}_{3} \mathrm{COCH}_{3}$ measurements. These losses were probably due to the slow collection of oil deposits on the grating or possibly gradual contamination of the end of the discharge caplllary tube. In the $\mathrm{CS}_{2}{ }^{+}$investigation, counts were accumulated for 200-300 seconds per data point at $1-2 \AA$ Intervals. Ion detection rates varied from aporoximately 45 counts/second at Iyman $\alpha$ to about 0.2 count/second at threshold. For the $\left(\mathrm{CS}_{2}\right)_{2}{ }^{+}$determination, counts were collected for 300-500 seconds per data point at $2.5 \AA$ intervals. Tyolcal counting rates ranged from 0.03 
count/second to 3 counts/second. Once again, a reference point was regularly repeated to assure overall calibration.

2. Results and Discussion

The monomer photolonization efficiency

curve is shown in Figure 22. Although wide monochromator entrance and exit slits were employed, the resolution of the data appears to be at least equivalent to that obtained in previous conventional photoionization studies at nominally higher resolutions (e.g., see Figure 5).122,305 This equivalence illustrates both the deleterious effects of sample internal energy in a room temperature experiment and the power of using molecular beam techniques to provide efficient thermal relaxation of the target molecules. The observed first ionization potential of $10.056 \pm 0.015 \mathrm{eV}$ is in excellent agreement with the earlier photolonization measurements. Values from vacuum ultraviolet absorption spectroscopy 303,304 are about $0.02 \mathrm{eV}$ higher. In addition, there are numerous apparent step-like fine structure features, especlally at $250-820 \mathrm{~cm}^{-1}$ and $1500 \mathrm{~cm}^{-1}$ above the initial onset. These features probably correspond to low energy vibrational excitations superimposed on structure due to a spin-orbit splitting of about $500 \mathrm{~cm}^{-1}, 122,123$ Unfortunately, a higher resolution measurement is necessary in order to make specific assignments. Regions of the $\mathrm{CS}_{2}{ }^{+}$ curve which contain sharp autoicnization peaks might aiso 


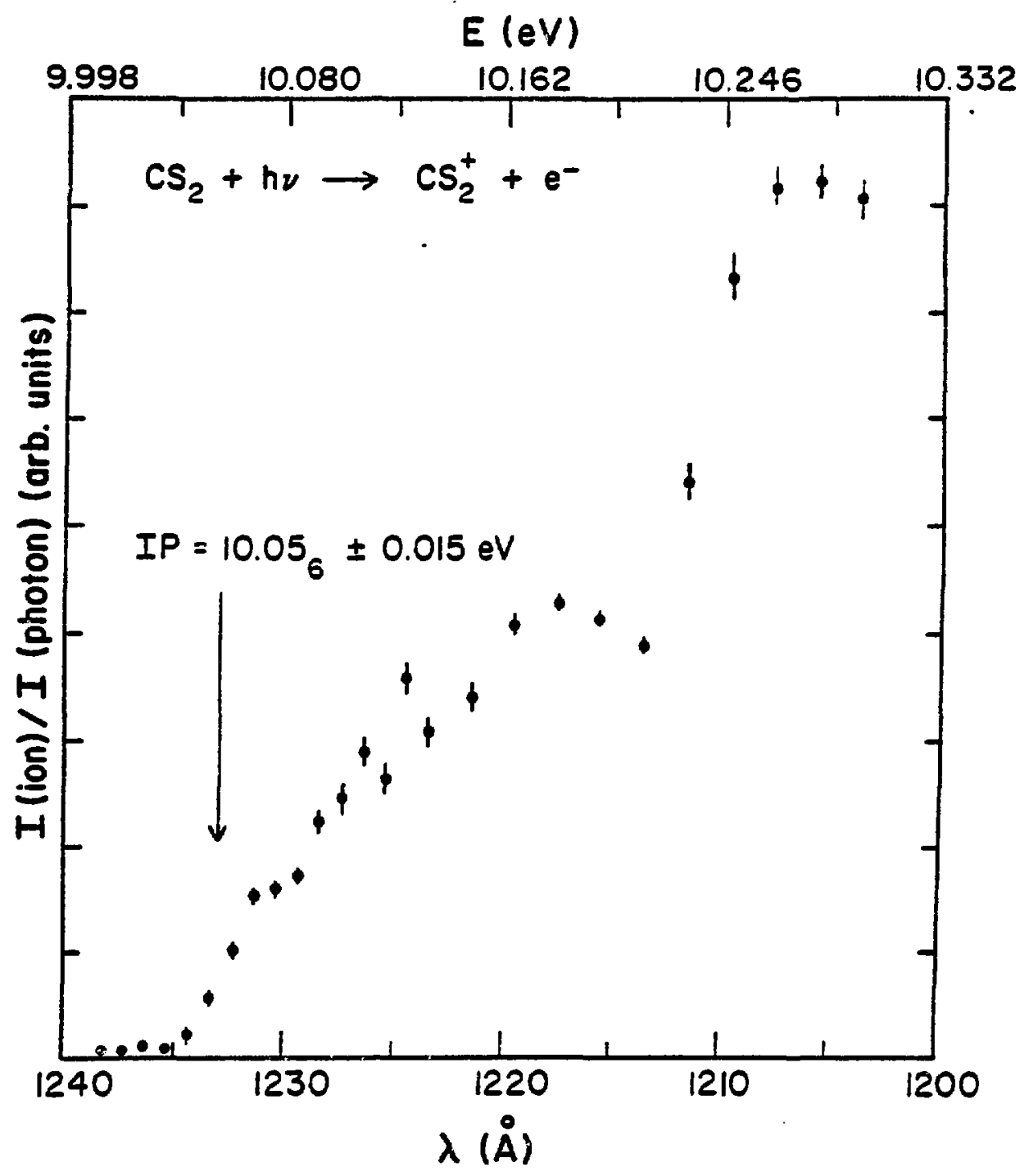

Figure 22.

Photoion Yield Curve for $\mathrm{CS}_{2}{ }^{+}$ from 1203 to 1239 \&. 
be profitably re-examined at the resolutions possible with molecular beam sampling. The value of further work on this system is clearly indicated.

The photolon yield curve for $\left(\mathrm{CS}_{2}\right)_{2}{ }^{+}$is shown in Figure 23. The most interesting portions of . this curve were measured at least twice. As in the case of $\left(\mathrm{CH}_{3} \mathrm{COCH}_{3}\right)_{2}^{+}$, the photolonization efficlency of $\left(\mathrm{CS}_{2}\right)_{2}^{+}$ rises very gradualiy from a weak onset. Within the sensitivity of the apparatus, the measured lonization potential for this specle is about $9.63 \mathrm{eV}$. Once again, the probability for the adiabatic transition may be so small as to prevent the observation of this onset above background. 83 A calculation of the dimer ion binding energy may be made in the manner described in Section II.B.3.C. by considering the following thermodynamic cycle:

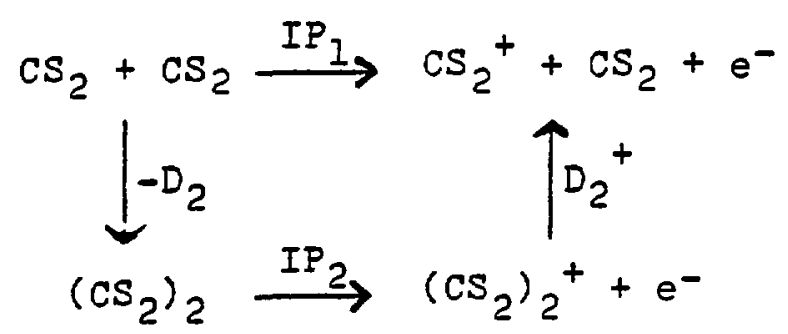

where $I P_{1}, I P_{2}, D_{2}$ and $D_{2}{ }^{+}$are defined as in Equation (24). Avallable second virial coefficlent $\left(B_{2}(T)\right.$ ) data for $\mathrm{CS}_{2}{ }^{293}$ may be employed to derive an estimate for $D_{2}$. These data and calculations are shown in Table $V$. Using the measured monomer and dimer ionlzation potentiais as well as 0.053 e? 


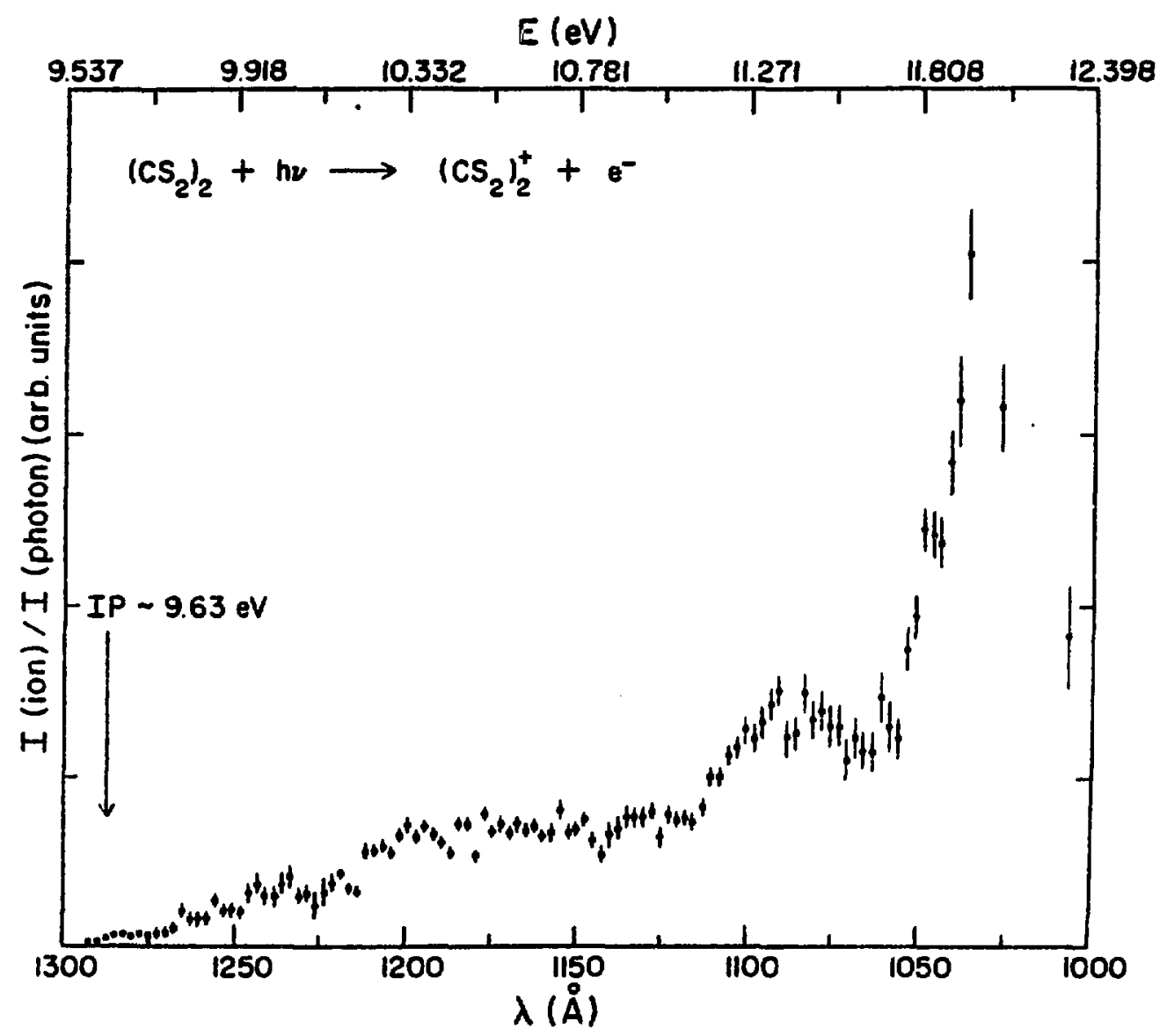

Figure 23.

$$
\begin{aligned}
& \text { Photolon Yleld Curve for }\left(\mathrm{CS}_{2}\right)_{2}{ }^{+} \\
& \text {from } 1007 \text { to } 1292 \AA \text {. }
\end{aligned}
$$


Table V. Cälulated Carbon Disulfide Dimer Binding Energy from $\mathrm{B}_{2}$ (T) Data. 293

\begin{tabular}{|c|c|c|c|c|}
\hline$T(K)$ & $1 / T\left(\times 20^{3}\right)$ & $-B_{2}(T)\left(\frac{c c}{m 01 e}\right)$ & $b_{0}-B_{2}(T)$ & in $\left[b_{0}-B_{2}(T)\right]$ \\
\hline 280 & 3.571 & $930 \pm 30$ & $1040 \pm 30$ & $6.9177-6.9470-6.975$ \\
\hline 290 & 3.448 & $862 \pm 30$ & $972 \pm 30$ & $6.8480-6.3794-6.9099$ \\
\hline 300 & 3.333 & $796 \pm 25$ & $906 \pm 25$ & $6.7811-6.8090-6.3363$ \\
\hline 310 & 3.226 & $740 \pm 20$ & $850 \pm 20$ & $5.7214-6.7452-6.7525$ \\
\hline 325 & 3.077 & $666 \pm 25$ & $776 \pm 15$ & $6.6346-6.6542-6.6733$ \\
\hline 350 & 2.857 & $567 \pm 15$ & $677 \pm 15$ & $6.4953-6.5177-6.5396$ \\
\hline 375 & 2.666 & $489 \pm 15$ & $\equiv 99 \pm 15$ & $6.3599-6.3953-5.4 \geq 20$ \\
\hline 400 & 2.500 & $429 \pm 15$ & $539 \pm 15$ & $6.2615-6.2897-6.3272$ \\
\hline 430 & 2.326 & $377 \pm 15$ & $487 \pm 15$ & $6.1570-6.2983-5.21 \geq 6$ \\
\hline
\end{tabular}

$b_{0}=110 \mathrm{cc} / \mathrm{mole}$

$b_{0}=2 / 3 \pi \sigma_{0}^{3}, \tilde{N}=$ Avogadro's number, $\sigma$ taken

from Table I-A in appendix of Reference 291)

From., temperature slope:

$$
\begin{aligned}
& \frac{d}{d\left(\frac{1}{T}\right)} \ln \left[E_{0}-B_{2}(\pi)=620 \mathrm{~K}\right. \\
& \begin{aligned}
D_{2}=\Delta u & =R \times 610 \mathrm{~K} \\
& =1.212 \mathrm{kcal} / \pi 0 \mathrm{ie} \\
& =0.053 \mathrm{eV}
\end{aligned}
\end{aligned}
$$


for the dimer binding energy, the above cycle gives a $\left(\mathrm{CS}_{2}\right)_{2}{ }^{+}$binding energy of $0.479 \mathrm{eV}$ or $11.0 \mathrm{kcal} / \mathrm{mole}$. In view of the uncertainty as to whether the adiabatic transition is observed for the dimer, this value must be consldered a lower bound.

A comparison of the $\left(\mathrm{CS}_{2}\right)_{2}{ }^{+}$photoicn yield curve with the $\left(\mathrm{CO}_{2}\right)_{2}{ }^{+}$data of Jones and Taylor 83,91 reveals some interesting simllarities. First, the measured dimer ionization potentials are shifted to lower energy by nearly the same amounts $\left(0.37 \mathrm{eV}\right.$ for $\mathrm{CO}_{2}$ dimer, $0.426 \mathrm{eV}$ for $\mathrm{CS}_{2}$ dimer) with both curves lacking sharo initial onsets. Second, the two sets of data predict dimer ion dissociation energies, $\mathrm{D}_{2}{ }^{+}$, which differ considerably from I1terature values. For instance, the calculated $\mathrm{D}_{2}{ }^{+}$for carbon disulfide dimer ion ( $11.0 \mathrm{kcal} / \mathrm{mole})$ is significantly below the enthalpy for the $\mathrm{CS}_{2}{ }^{+}-\mathrm{CS}_{2}$ ion-molecule association reaction $\left(-\Delta H^{\circ}=21.9 \pm 2.5 \mathrm{kcal} / \mathrm{mole}\right)$ recently determined by Meot-Ner and Field 312 using pulsed high pressure mass spectrometry. At the same time, the data of Jones and Taylor would appear to indicate a $\mathrm{D}_{2}{ }^{+}$for $\left(\mathrm{CO}_{2}\right)_{2}{ }^{+}$ in the range of $9-11 \mathrm{kcal} / \mathrm{mole}$. The enthalpy for the $\mathrm{CO}_{2}^{+}-\mathrm{CO}_{2}$ ion clustering reaction is once again much higher $\left(-\Delta \mathrm{H}^{\circ}=16.2 \pm 1.5 \mathrm{kcal} / \mathrm{mole}\right) .312$ These discrepancies may be due to the Inability to observe the $\mathrm{v}^{\prime}=0 \rightarrow \mathrm{v}^{\prime}=0$ transition sor the dimen; heveron, the possibility of 
error in the ion-molecule reaction studies (1.e., van't Hoff plots) must also be considered. Finally, the $\left(\mathrm{CS}_{2}\right)_{2}{ }^{+}$and $\left(\mathrm{CO}_{2}\right)_{2}{ }^{+}$photolonization efficiency curves are similar in terms of fine structure. In each case, the dimer curve exhibits one or more autolonization features which resemble peaks occurring in the monomer curve at nearly identical photon energies. By the same token, some fine structure appears to be lost or at least obscured in going from the monomer to the dimer in both systems (e.g., compare the 1050-1150 \& wavelength region in Figures 5 and 23). One detail which seems to be different in the two sets of data is the fact that the most intense autolonization peak in the carbon dioxide curves is much broader for the dimer whereas the peak at 1000-1050 $\AA$ in the curves of the carbon disulfide species shows very little, if any, change in width. Observed peak broadening of this type could result from poorer resolution in the dimer studies or possibly from a decrease in the ilfetime of the exclted Rjoberg-like states in going from monomer to dimer.

It should be noted that an interesting application may result from the knowledge of relative peak positions in photolon yield curves of such fons as $\mathrm{CS}_{2}{ }^{+}$ and $\left(\mathrm{CS}_{2}\right)_{2}{ }^{+}$. If the initial excitation step of an Individual autoionizinz transitizn can be correiated 
between the parent and cluster molecules (i.e., a given resonance process in the dimer, trimer, etc. can be considered to be identical to a corresponding excitation In the monomer, except for a slight perturbation due to the presence of other monomeric units), the following cycle may be employed:

$$
\begin{gathered}
A B+A B \stackrel{E_{1}}{\longrightarrow} A B^{*}+A B \\
\downarrow-D_{2} \\
(A B)_{2} \stackrel{E_{2}}{\longrightarrow}{ }_{(A B)_{2}}^{*}
\end{gathered}
$$

where $E_{1}$ and $E_{2}$ are the photon energies at which the similar peaks are observed and $D_{2}{ }^{*}$ is the dissociation $\epsilon$ nergy of the process shown. An estimate or measurement of $D_{2}$ coupled with the known values of $E_{1}$ and $E_{2}$ allows the determination of $\mathrm{D}_{2}{ }^{*}$. In order to apply the cycle to $\mathrm{CS}_{2}$ and $\left(\mathrm{CS}_{2}\right)_{2}$, It would be necessary to oerform more careful measurements on both species; however, a cursory examination of the available data (using Figure 5) indicates that $D_{2}$ and $D_{2}{ }^{*}$ are very similar. 


\section{RECOMMENDATIONS}

With the molecular beam photolonization mass spectrometer as constructed, it is possible to investigate at high resolution the photolonization efficlency behavior of a large number of parent, cluster and fragment lons. Several proposed experiments will be discussed in the present section. Before dealing with this subject, however, a number of possible developments in the instrumental design w1ll be examined. Indeed, there are many improvements which could be made in order to simplify the data collection, increase the photon energy resolution and enhance the ion transmission and detection properties of the mass analyzer. In addition, it would be desirable to extend the energy range of the apparatus so as to possess the capability of study Ing a wider variety of compounds.

Two fairly simple modifications would serve to make the data collection more convenient. First, it would be helpful to integrate and digitize the voltage output of the picoammeter used to monitor the intensity of the dispersed vacuum ultraviolet light. This step would require an amplifier integrator, together with the appropriate gating circuitry and a provision for displaying and clearing the output. .Instrumentation of this 
type would eliminate the need to constantly monitor the plcoammeter output and would automatically account for any slgnificant short-term fluctuations in lamp intensity. Second, a substantial amount of time could be saved if the entire data collection system were automated. At present, the monochromator grating drive and ion counter are operated manually. Thus, an automated operatlonal mode would involve coupling a stepping motor to the wavelength scan mechanism of the monochromator and providing the necessary circuitry to print out the ion count and integrated photon output after a variable preset time. Upon printing the signals, data collection could immediately proceed by stepping the monochromator to a new wavelength and resetting and restarting the scalers. The long-term stability of the lamp could be monltored by passing the voltage output of the picoammeter to an $x-y$ recorder.

It need hardly be stated that improved mass selected Ion transmission and detection would be advantageous in terms of reducing the time required per data point and allowing the investigation of molecular beam constituents which : re present in low concentrations (e.g., larger clusters). Despite the discrepancy between the calculated collection efficiency of the present apparatus and 
reported values for a similar instrument, 55,140 it is unclear at this point how the mass spectrometer transmission could be substantially increased inasmuch as several ion box and fon extraction slit designs have been tried with only small observed changes in signal. Also, a check on the mass analyzer alfgnment indicated that this aspect was falriy well optimized. On the other hand, it would be useful to improve the fon detection system by eliminating the need for the 20db attenuator in the data collection electronics. This step alone would Increase the count rate by a factor 0 ? about 2.5. In this regard, it is expected that significantly reduced nolse levels can be achleved by providing better shielding for the election multiplier tube and voltage divider arrangement and also by passing the voltage pulse produced at the anode of the multiplier through an electronically isolated connector (i.e., eliminating ground loops).

In spite of the fact that the photoionization mass spectrometer was largely assembled from available surplus equipment, most of the basic instrumentation is quite adequate. However, one major item which could concelvably be replaced is the vacuum ultraviolet monochromator. As mentioned in Section II.D., near-normal inciderce monochromators are much better than 
Seya-Namioka type instruments in terms of dispersed light throughput and ultimate resolution. Unfortunately, such a change would necessitate redesigning the differentlal pumping system. The high cost of a $1-\mathrm{m}$. or 3-m. near-normal incidence device is also a negative factor. In addition, space limitations in the present orlentation of the apparatus would be severe enough that the whole instrument would have to be moved and reallgned.

W1th or without a new monochromator, a modified vacuum system with much hizher pumping speed would be advantageous. In particular, it would be useful to have the capacity for handling the increased gas loads associated with intense helium capillary discharges. The Ionization and dissociative properties of many interesting compounds may be studied with the He Hopfleld continuum (580-1100 \&); however, relatively high lamp pressures (40-100 torr) are required. Reasonable modifications in the vacuum system might include the use of a larger Roots blower on the first differential pumping chamber and possibly the incorporation of another differential pumping region in the vicinity of the monochromator exit slit. Since the Hopfield continuum is sufficiently excited only in a repetitive discharge, aditsunal electronies rouid be heeded incilidne a hish 
voltage pulser and an appropriate power supply (about $15 \mathrm{kV}, 500 \mathrm{~mA})$. For best results below $900 \AA$, a gold or platinum coated grating with a suitable blaze angle. would be employed.

Two possible additions to the nozzle beam sampling system should be briefly mentioned. First, the installation of a time-of-flight correlation chopper would be useful in providing a direct measure of molecular beam temperatures. At the present time, crude temperature estimates may be made only by examining the energy spread of step-like thresholds in the photoion yleld curves. Second, enhanced moromer and cluster beam Intensities might be realized if the primary skimmer and endwall were cryocooled (see Figure 7). Clogging of the skimmer can be a severe problem in such designs, however. This problem might be especially troublesome in photoIonization experiments, since long data collection times are typically required.

The photoionization efficiency curves of a conslderable number of lons have been determined using supersonic molecular beam photoionization mass spectrometry. These ions are listed in Table VI according to the investigating group. Many very interesting systems remain to be studied, however. For instance, the present work 
Table VI. Ions For Which Photolonization Efficiency Curves Have Been Determined Using Supersonic Molecular Beam Mass Spectrometry.

\section{Investigating Group}

1. James $w$. Taylor et. al., $79-31,83,91,92$ U. of Hisconsin U. of Calffornia, Berke?ey
2. Yuan T. Lee, et. aI. $82,84-90,117$

Soecies Studied

$\mathrm{CO}_{2}^{+},\left(\mathrm{CO}_{2}\right)_{2}^{+}, \mathrm{CH}_{3} \mathrm{OH}^{+},\left(\mathrm{CH}_{3} \mathrm{OH}\right)_{2^{+}}$

$\mathrm{NO}^{+},(\mathrm{NO})_{2}^{+}, \mathrm{Xe}_{2}^{+}, \mathrm{Ar}_{2}^{+}, \mathrm{Mr}_{2}^{+}$, $\mathrm{XeKr}+, \mathrm{KeAr} \mathrm{KrAr}^{+}, \mathrm{KrI}^{+}$, ArICI ${ }^{+}, \mathrm{CH}_{3} \mathrm{I}^{+}, 3_{3}^{+}, \mathrm{Z}_{2} \mathrm{O}^{+}, \mathrm{Z}_{3} \mathrm{O}^{+}$, $\left(\mathrm{H}_{2} \mathrm{O}\right)_{2}^{+}, \mathrm{NH}_{3}^{+},\left(\mathrm{NH}_{2}\right)_{2}^{+},\left(\mathrm{NH}_{2}\right)_{\mathrm{n}^{+}}$, $\mathrm{HF}^{+},(\mathrm{HF})_{\mathrm{n}^{+}}, \mathrm{HCl}^{+}, \mathrm{r}_{2} \mathrm{CI}^{+}$, $(\mathrm{HCl})_{2}^{+}, \mathrm{C}_{2} \mathrm{ri}_{4}^{+},\left(\mathrm{C}_{2} \ddot{\ddot{z}}_{4}\right)_{2}^{+}, \Sigma_{4} \ddot{z}_{7}^{+}$, $\mathrm{C}_{3} \mathrm{~F}_{5}^{+}$

3. This work.
$\mathrm{CH}_{3} \mathrm{COCH}_{3}^{+}, \mathrm{CD}_{3} \mathrm{COCO}_{3}^{+}, \mathrm{OH}_{3} \mathrm{CO}^{+}$,

$\mathrm{CD}_{3} \mathrm{CO}^{+}, \quad\left(\mathrm{ZH}_{3} \mathrm{COCH}_{3}\right)^{+}, \mathrm{n=}=3-4$,

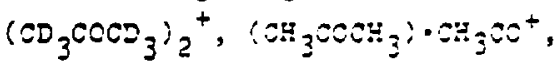

$\mathrm{Cs}_{2}^{+},\left(\mathrm{Cs}_{2}\right)_{2}^{+}$ 
could be extended by carefully examining the photolon yleld behavior of $\mathrm{CS}_{2}{ }^{+}$from about 900 \& to threshold. At high resolution, it should be possible to identify individual vibrational excitations near the first onset and also to precisely measure the positions of autoIonization peaks. A better comparison of the fine structure in the monomer and dimer curves could be made. If the intensity of either the dispersed vacuum ultraviolet light or clusters in the molecular beam can be increased, photoionization efficiency curves of $\left(\mathrm{CH}_{3} \mathrm{COCH}_{3}\right)_{n}^{+}, \mathrm{n}=5,5,7 \ldots$ could be determined. These measurements would be especlally useful in relation to the information contained in Figure 21 . The invest1gation of Ionization and fragmentation oroperties of a wide varlety of other compounds is feasible as well. With the $\mathrm{H}_{2}$ many-line pseudo-continuum, parent and cluster species in nozzle beams of $\cos$, cyclopropane, propylene, I, 3-butadiene, $\mathrm{Cl}_{2}, \mathrm{Br}_{2}, \mathrm{CH}_{3} \mathrm{Cl}, \mathrm{CH}_{2} \mathrm{Cl}_{2}, \mathrm{H}_{2} \mathrm{~S}, \mathrm{CH}_{3} \mathrm{SH}$, etc. may be studied. Experiments on clusters of such aromatic molecules as benzene, hexafluorobenzene and toluene could be potentially very intriguing in comparison with recent electric dipole moment determinations for these compounds. 313,314 In addition, if the monochromator vacuum system can be upgraded and the necessary electronics 
obtalined to produce a pulsed He discharge, the photolon yleld curves of $\mathrm{CO}^{+}, \mathrm{SF}_{5}{ }^{+}, \mathrm{UF}_{5}{ }^{+}$and many other interesting Ions could be measured.

For molecules such as $\mathrm{H}_{2} \mathrm{~S}$, molecular beam photoIonization studies can lead to the determination of proton affinities (PA) and proton solvation energies (SE). Investigations of this type have already been performed on $\mathrm{H}_{2} \mathrm{O}$, HF and $\mathrm{NH}_{3} .84$ To calculate $\mathrm{PA}$ for hydrogen sulfide, the following thermodynamic cycle may be used:

$$
\begin{aligned}
& \mathrm{H}_{2} \mathrm{~S}+\mathrm{H}_{2} \mathrm{~S} \stackrel{\mathrm{AP}_{I}}{\longrightarrow} \mathrm{H}_{2} \mathrm{~S}+\mathrm{H}^{+}+\mathrm{SH}+e^{-} \\
& \downarrow-D_{2} \quad \uparrow-P A \\
& \left(\mathrm{H}_{2} \mathrm{~S}\right)_{2} \stackrel{\mathrm{AP}_{2}}{\longrightarrow} \mathrm{H}_{3} \mathrm{~S}^{+}+\mathrm{SH}+\mathrm{e}^{-}
\end{aligned}
$$

where $A P_{1}, A P_{2}$ and $D_{2}$ are defined as before (see Equation 31). Since $\mathrm{AP}_{1}$ and $\mathrm{AP}_{2}$ can be directly measured, an estimate of $D_{2}$ ylelds the proton affinity. Appearance potential determinations for the dissociative ionization of hydrogen sulfide trimers, tetramers, etc. to form $\left(\mathrm{H}_{2} \mathrm{~S}\right)_{\mathrm{n}} \mathrm{H}^{+}$may also be utilized in approprlate thermochemical cycles such as

$$
\begin{aligned}
& \left(\mathrm{H}_{2} \mathrm{~S}\right)_{\mathrm{n}}+\mathrm{H}_{2} \mathrm{~S} \stackrel{\mathrm{AP}_{\mathrm{n}}}{\longrightarrow} \mathrm{H}_{2} \mathrm{~S}+\left(\mathrm{H}_{2} \mathrm{~S}\right)_{\mathrm{n}-\mathrm{I}} \mathrm{H}^{+}+\mathrm{SH}+\mathrm{e}^{-} \\
& \downarrow-D_{n+1} \quad \uparrow-S E_{n-1, n} \\
& \left(\mathrm{H}_{2} \mathrm{~S}\right)_{\mathrm{n}+\mathrm{I}} \stackrel{\mathrm{AP}_{\mathrm{n}+1}}{\longrightarrow}\left(\mathrm{H}_{2} \mathrm{~S}\right)_{\mathrm{n}} \mathrm{H}^{+}+\mathrm{SH}+\mathrm{e}^{-}
\end{aligned}
$$


where $S E_{n-1, n}$ represents the solvation energy corresponding to the addition of the $n$th $\mathrm{H}_{2} \mathrm{~S}$ constituent.

For $n=2-5$, these enthalples can be compared to avallable literature values. 315 Analogous experiments could be performed on $\mathrm{D}_{2} \mathrm{~S}$.

The photolonization efficlency behavior of many interesting mixed cluster systems remain to be explored. For example, the enthaligy $\left(\Delta_{\mathrm{H}_{3}} \mathrm{~S}^{+} \cdot \mathrm{H}_{2} \mathrm{O}\right)$ for the clusterlng reaction

$$
\mathrm{H}_{3} \mathrm{~S}^{+}+\mathrm{H}_{2} \mathrm{O} \rightleftharpoons \mathrm{H}_{3} \mathrm{~S}^{+} \cdot \mathrm{H}_{2} \mathrm{O}
$$

has been reported. 315 since mixed clusters such as $\left(\mathrm{H}_{2} \mathrm{~S}\right)_{2} \cdot \mathrm{H}_{2} \mathrm{O}$ can be produced in a supersonic expansion, It shorid be possible to measure the appearance potential (AP') of $\mathrm{H}_{3} \mathrm{~S}^{+} \cdot \mathrm{H}_{2} \mathrm{O}$ and then calculate the $\left(\mathrm{H}_{2} \mathrm{~S}\right)_{2} \cdot \mathrm{H}_{2} \mathrm{O}$ binding energy $\left(-D_{2,1}\right)$ from the following cycle:

$$
\begin{aligned}
& \left(\mathrm{H}_{2} \mathrm{~S}\right)_{2}+\mathrm{H}_{2} \mathrm{O} \stackrel{\mathrm{AP}_{2}}{\longrightarrow} \mathrm{H}_{3} \mathrm{~S}^{+}+\mathrm{H}_{2} \mathrm{O}+\mathrm{SH}+\mathrm{e}^{-} \\
& \downarrow-\mathrm{D}_{2,1} \uparrow-\Delta \mathrm{H}_{\mathrm{H}_{3} \mathrm{~S}}{ }^{+} \cdot \mathrm{H}_{2} \mathrm{O} \\
& \left(\mathrm{H}_{2} \mathrm{~S}_{2} \cdot \mathrm{H}_{2} \mathrm{O} \stackrel{\mathrm{AF}^{\prime}}{\longrightarrow} \mathrm{H}_{3} \mathrm{~S}^{+} \cdot \mathrm{H}_{2} \mathrm{O}+\mathrm{SH}+\mathrm{e}^{-}\right.
\end{aligned}
$$

where $\mathrm{AP}_{2}$ may be directly measured as in Equation (93). Once again, these experiments could be extended to higher clusters and other 1sotopes. As another example, photoIonization studies of such polymers as $\mathrm{NO} \cdot\left(\mathrm{H}_{2} \mathrm{O}\right)_{n}$, $\mathrm{O}_{2} \cdot\left(\mathrm{H}_{2} \mathrm{O}\right)_{\mathrm{n}}$, $\mathrm{NO} \cdot \mathrm{CO}_{2}$ and $\mathrm{NO} \cdot \mathrm{N}_{2}$ could be particularly useful inasmuch as their respective ions... $\mathrm{NO}^{+}\left(\mathrm{H}_{2} \mathrm{O}\right)_{2}, \mathrm{O}_{2}{ }^{+}\left(\mathrm{H}_{2} \mathrm{O}\right)_{n}$, etc.) are involved in principal chemical reactions in the 
D region of the 1onosphere. 316,317 In spite of the vital role which these ions play, very little is known about their vibrational spectra, structures or thermochemistry. It is precisely this type of information which could be at least partially obtained in a sufficiently high resolution photolonization experiment.

Finally, the rapid development of high power lasers of various kinds makes it reasonable to speculate on future utilization of this burgeoning technology in molecular beam photolonization mass spectrometric studies. Needless to say, the most straightforward application would involve the design of tunable high intensity vacuum ultraviolet cw lasers and the use of such instruments as photoionization light sources. Another possible application would be the study of Ionization and fragmentation processes using target molecules which are predominantly in excited electronic or vibrational states. The inftial excitation step could be accomplished by a laser of sultable wavelength whlle another laser (tunable) or a conventional light source could be used for the photolonization process. 


\section{References}

I. H. M. Rosenstock, Int. J. Mass Spect. Ion Phys. 20, 139 (1976).

2. J. H. Beynon, R. G. Cooks, R. K. Jennings and A. J. Ferrer-Correfa, Int. J. Mass Spect. Ion Phys. 18, 87 (1975).

3. J. H. D. Eland, "Photoelectron Spectroscopy," (Butterworths \& Co., Ltd., London, 1974), p. 56.

4. M. E. Wacks, in Modern Aspects of Mass Spectrometry," R. I. Reed, Ed., (Plenum Press, New York, 1968), p. 323 .

5. J. E. Collin, in "Mass Spectrometry," R. I. Reed, Ed., (Academic Press, New York, 1965), p. 183.

6. M. Krauss and V. H. Dibeler, in "Mass Spectrometry of Organic Ions," F. W. McLafferty, Ed., (Academic Press, New York, 1963), p. 117.

7. C. A. MeDowell, in "Mass Spectrometry," C. A. McDowell, Ed., (McGraw-Hill, San Francisco, Callfornia, 1963), p. 506.

8. H. M. Rosenstock, K. DraxI, J. T. Herron and $B$. Stelner, J. Phys. Chem. Reference Data $\underline{6}$, Supplement I, 1977.

9. A. B. F. Duncan, "Rydberg Series in Atoms and Molecuies," (Acajemic Press, ":en Fonk, 1971). 
10. Ib1d., p. 80

11. A. B. F. Duncan, J. Chem. Phys. 3, 131 (1935).

12. K. Watanabe, J. Chem. Phys. 22, 1564 (1954).

13. I.-J. Kang and W. D. Foland, Phys. Rev. 164, 122 (1967).

14. R. W. Ditchburn and U. Öpik in "Atomic and Molecular Processes," D. R. Bates, Ed., (Academic Press, New York, 1962), p. 79.

15. S. Geltman, Phys. Rev. 102, 171 (1956).

16. G. H. Wannier, Phys. Rev. 100, I180 (1955).

17. H. S. W. Massey and E. H. S. Burhop, "Ele atronic and Ionfc Impact Phenomena," (Oxford Universtty Press, London, 1952).

18. E. Wigner, Phys. Rev. 73, 1002 (1948).

19. F. H. Field and J. L. Franklin, "Electron Impact Phenomena and the Properties of Gaseous Ions," (Academic Press, New York, 1957).

20. B. M. Johnson and J.W. Taylor, Int. J. Mass Spect. Ion Phys. 10, 1 (1972).

21. G. G. Melsels, C. T. Chen, B. G. Glessner and R. H. Emmel, J. Chem. Phys. 56, 793 (1972).

22. C. E. Brion ara L. D. Hall, J. Amer. Chem. Soc. 88, 3361 (1966).

23. J. D. Morrison, in "Mass Spectrometry," A. Maccoll, Ed., (Buttervorths \& co., Ėd., Iondon, 2972), 0.25 . 
24. J. E. Collin, in "Modern Aspects of Mass Spectrometry," R. I. Reed, Ed., (Plenum Press, New York, 1968), p. 231.

25. A. Kuppermann, J.K. Rice and S. Trajmar, J. Phys. Chem. 12,3894 (1968).

26. J. Wayne Rabala1s, "Principles of Ultraviolet Photoelectron Spectroscopy," (John W1ley \& Sons, New York, 1977).

27. C. R. Brundle and A. D. Baker, Ed., "Electron Spectroscopy: Theory, Techniques and Applications," (Academ1c Press, New York, 1977).

28. Thomas A. Carlson, "Photoelectron and Auger Spectroscopy," (Plenum Press, New York, 1975).

29. A. D. Baker and D. Betteridge, "Photoelectron Spectroscopy: Chemical and Analyt1cal Aspects," (Pergamon Press, Oxford, 1972).

30. "The Photoelectron Spectroscopy of Molecules," Faraday Discuss. Chem. Soc. London, 54, I (1972).

31. J. E. Collin, in "Recent Topics in Mass Spectrometry," R. I. Reed, Ed., (Gordon and Breach Science Publishers, New York, 1971).

32. R. Spohr, P. M. Guyon, W. A. Chupka and $J$. Berkow1tz, Rev. Sc1. Instr. 42, 1872 (1971). 
33. D. W. Turner, C. Baker, A. D. Baker and C. R. Brundle, "Molecular Photoelectron Spectroscopy:

A Hanabook of He 584 \& Spectra," (Wiley-Interscience, New York, 1970).

34. D. R. Lloyd, J. Phys. E 3, 629 (1970).

35. S. Geltman, Phys. Rev. 112, 176 (1958).

36. K. Watanabe, J. Chem. Phys. 26, 542 (1957).

37. James A. R. Samson, Physics Reports 28, 303 (1976).

38. N. W. Reld, Int. J. Mass Spec. Ion Phys. 6, I (1971).

39. F. $J$. Comes, in "Advances in Mass Spectrometry," Vo1. 4, E. Kendrick, Ed., (The Institute of Petroleum, London, 1968), p. 737.

40. A. L. Hughes and L. A. DuBridge, "Photoelectric Phenomena" (McGraw-Hill, New York, 1932).

41. K. Watanabe, T. Nakayama and J. Mottl, "Final Report on Ionization Potential of Molecules by a PhotoIonization Method," AD-231 889, 1959.

42. R. W. Ditchburn and F. L. Arnot, Proc. Roy. Soc. A123, 516 (1929).

43. A. Terenin and B. Popov, Phys. Z. Sowjetunion 2, 299 (1932).

44. D. M. Packer and C. Lock, J. Opt. Soc. Amer. 4I, 699 (1951).

45. K. Watanabe, T. Nakayama and J. MottI, J. Quant. Spect. Radiat. Transfer 29350 (2902). 
46. D. J. Knowles and A. J. C. Nicholson, J. Chem. Phys. 60, 1180 (1974).

47. I. P. Lossing and I. Tanaka, J. Chem. Phys. 25, 1031 (1956).

48. R. F. K. Herzog and F. F. Marmo, J. Chem. Phys. 27, 1202 (1957).

49. H. Hurzeler, M. G. Inghram and J. D. Morrison, J. Chem. Phys. 27, 313 (1957).

50. H. Hurzeler, M. G. Inghram and J. D. Morrison, J. Chem. Phys. 28, 76 (1958).

51. G. I. Welssler, J. A. R. Samson, M. Ogawa and G. R. Cook, J. Opt. Soc. Amer. 49, 338 (1959).

52. D. C. Frost, D. Mak and C. A. McDowell, Can. J. Chem. 40, 1064 (1962).

53. F. J. Comes and W. Lessman, Z Naturforschg 19a, 65 (1964).

54. W. P. Poschenrieder and P: Warneck, J. Appl. Phys. 37, 2 2 12 (1966).

55. J. Berkowitz and W. A. Chupka, J. Chem. Phys. 45, 1287 (1966).

56. V. H. Dibeler, R. M. Reese and M. Krauss, in "Advances in Mass Spectrometry," Vol. 3, W. L. Mead, Ed., (The Institute of Petroleum, London, 1966), p. 471. 
57. F. A. Elder, D. Villarejo and M. G. Inghram, J. Chem. Phys. $\underline{43}, 758$ (1965).

58. I. Omura, T. Kaneko, Y. Yamada and T. Kondo, Mass Spectroscopy 16, 349 (1968).

59. J. Momigny, C. Goffart and I. D'Or, Int. J. Mass Spect. and Ion Phys. I, 53 (1968).

60. A. J. C. Nicholson, Vacuum 18, 675 (1968).

61. P. J. Foster, R. E. Leckenby and E. J. Robbins, J. Phys. B. 2 , 478 (1969).

62. D. Reinke, R. Kräbig and H. Baumgärtel, Z. Naturforschg. 28a, 1021 (1973).

63. R. Browning and J. Fryar, J. Phys. B. 5, 364 (1973).

64. P. R. LeBreton, A. D. Wliliamson, J. L. Beauchamp and W. T. Huntress, J. Chem. Phys. 62, 1623 (1975).

65. P. M. Dehmer and W. A. Chupka, J. Chem. Phys. 65, $2243(1976)$.

66. W. A. Chupka, J. Chem. Phys. 54, 1936 (1971).

67. P. M. Guyon and J. Berkowitz, J. Chem. Phys. 54, 1814 (1971).

68. W. A. Chupka, J. Chem. Phys. 48, 2337 (1968).

69. W. A. Chupka, J. Chem. Phys. 30, 191 (1959).

70. P. M. Dehmer and W. A. Chupka, J. Chem. Phys. 66, 1972 (1977).

71. W. A. Chupka, P. M. Dehmer and W. T. Jivery, J. Chem. Phys. E3, 3929 (1975). 
72. P. M. Dehmer and W. A. Chupka, J. Chem. Phys. 62, 4525 (1974).

73. K. E. McCulioh, J. Chem. Phys. 59, 4250 (1973).

74. V. H. Dibeler and J. A. Walker, Int. J. Mass Spect. and Ion Phys. 11, 49 (1973).

75. V. H. Dibeler, J. A. Walker, K. E. McCulloh and H. M. Rosenstock, Int. J. Mass Spect, and Ion Phys. I, 209 (1971).

76. J. Berkow1tz, W. A. Chupka, P. M. Guyon, J. H. Holloway and R. Spohr, J. Chem. Phys. 54, 5165 (1971).

77. V. H. Dibeler, J. A. Walker and K. E. MeCulloh, J. Chem. Phys. 53, 4414 (1970).

73. W. A. Chupka and J. Berkow1tz, J. Chem. Phys. 51, 4244 (1969).

79. G. R. Parr and J.W. Taylor, Rev. Sc1. Instr. 44, 1578 (1973).

80. G. R. Parr, Ph. D. Thesis, The University of Wisconsin, 1973.

81. G. R. Parr and J.W. Taylor, Int. J. Mass Spect. and Ion Phys. 14, 467 (1974).

82. P. W. Tiedemann, S. T. Ceyer, C. $-Y$. Ng, D. J. Trevor, P. I. Kronebusch, B. H. Mahan and Y. T. Lee, "Energetics of Molecule Ions by the Molecular Beam Photolonization Method," 175th ACS National Meeting, Anaheim, Calif., March 13-i7, i973. 
83. G. G. Jones and J. W. Taylor, J. Chem. Phys. 68, 1768 (1978).

84. C.-Y. Ng, D. J. Trevor, P. W. Tledemann, S. T. Ceyer, P. L. Kronebusch, B. H. Mahan and Y. T. Lee, J. Chem. Phys. 67, 4235 (1977).

85. C.-Y. Ng, P. W. Tledemann, B. H. Mahan and Y. T. Lee, J. Chem. Phys. 66, 5737 (1977).

86. C.-Y. Ng, P. W. Tiedemann, B. H. Mahan and Y. T. Lee, J. Chem. Phys. 66, 3985 (1977).

87. C. - Y. Ng, D. J. Trevor, B. H. Mahan and Y. T. Lee, J. Chem. Phys 66, 446 (1977).

88. C.-Y. Ng, D. J. Trevor, B. H. Mahan and Y. T. Lee, J. Chem. Phys $\underline{65}, 4327$ (1976).

89. C.-Y. Ng, B. H. Mahan and Y. T. Lee, J. Chem. Phys. 65, 1956 (1976).

90. C.-Y. Ng, Ph.D. Thesis, The University of California, Berkeley, 1976.

91. G. G. Jones, Ph.D. Thes1s, The University of Wisconsin, 1975.

92. J. W. Taylor, G. R. Parr and G. G. Jones, "PhotoIonization Mass Spectrometry Using Molecular Beam Sampling and Synchrotron Radlation," Vac. Ultraviolet Radiat. Phys., Proc. Int. Conf., 4th 1974, p. 197. 
93. G. Herzberg, "Molecular Spectra and Molecular Structure III. Elsctronic Spectra and Electronic Structure of Polyatomic Molecules" (Van Nostrand, Princeton, N. J., 1966).

94. H. M. Rosenstock and R. Botter, in "Recent Developments in Mass Spectroscopy," K. Ogawa and T. Hayakowa, Eds. (University Park Press, Balt1more, 1970), p. 797.

95. S. E. Schvartz, J. Chem. Educ. 50, 608 (1973).

96. H. A. Jahn and E. Teller, Proc. Roy. Soc. 161A, 220 (1937).

97. G. Herzberg and E. Teller, Z. Phys. Chem. B2I, 410 (1933).

98. R. Renner, Z. Phys. 92, 172 (1934).

99. G. Wentzel, Z. Phys. 43, 524 (1927).

100. G. Wentzel, Physik Z. 29, 321 (1928).

101. W. A. Chupka, in "Ion-Molecule Reactions," Vol. I, J. L. Franklin, Ed., (Plenum Press, New York, 1972), p. 33.

102. U. Fano, Phys. Rev. 124, 1866 (1961).

103. U. Fano and J. W. Cooper, Phys. Rev. AI37, 1364 (1965).

104. U. Fano and J. W. Cooper, Rev. Mod. Phys. 40, 441 (1968). 
105. F. H. Mies, Phys. Rev. 175, 164 (1968).

106. J. N. Bardisley, Chem. Phys. Letters 2, 329 (1968).

107. A. I. Sm1th, Ph1l. Trans. Roy. Soc. London A268, 169 (1970).

108. B. R1tch1e, Phys. Rev. A6, 1761 (1972).

109. B. Ritchle, Phys. Rev. A3, 95 (1971).

110. R. S. Berry and S. E. Nielsen, Phys. Rev. Al, 395 (1970).

111. S. E. Nielsen and R. S. Berry, Chem. Phys. Letters 2, 503 (1958).

112. A. Russek, M. R. Patterson and R. I. Becker, Phys. Rev. 167, 17 (1968).

113. J. N. Bardsley, Chem. Phys. Letters I, 229 (1967).

114. R. S. Berry, J. Chem. Phys. 45, 1228 (1966).

115. J. Berkowitz and W. Chupka, J. Chem. Phys. 51, 2341 (1969).

116. W. A. Chupka and J. Berkow1tz, J. Chem. Phys. 48, 5727 (1968).

117. E. Mlescher, Y. T. Lee and P. Gürtler, J. Chem. Phys. 68, 2753 (1978).

118. J. F. Lowry, D. H. Tomboulian and D. L. Ederer, Phys. Rev. AI37, 1054 (1965).

119. P. H. Krupenie, J. Phys. Chem. Ref. Data I, 423 (1972). 
120. V. H. Dibeler, J. A. Walker and H. M. Rosenstock J. Res. NBS 70A, 459 (1966).

121. P. C. K1llgoar, Jr., G. E. Lero1, W. A. Chupka and J. Berkowitz, J. Chem. Phys. 59, 1370 (1973).

122. V. H. Dibeler and J. A. Walker, J. Opt. Soc. Amer. 57, 1007 (1967).

123. R. Frey, B. Gotchev, W. B. Peatman, H. Pollak and E. W. Schlag, Int. J. Mass Spect. Ion Phys. 26, 137 ( 1978 ).

124. J. H. D. Eland and C. J. Danby, Int. J. Mass Spect. Ion Phys. I, 111 (1968).

125. A. J. C. Nicholson, J. Chem. Phys. 43, 1171 (1965).

126. "Handbook of Diffraction Gratings Ruled and Holographic," Jobin-Yron Optical Systems, Metuchen, N.J.

127. H. M. Rosenstock, M. B. Wallenstein, A. L. Wahrhaftig and H. Eyring, Proc. Natl. Acad. Sci. U.S. 38, 667 (1952).

128. H. M. Rosenstock and M. Krauss, in "Advances in Mass Spectrometry," Vol. 2, R. M. Ell1ott, Ed., (Macmilian, New York, 1963), p. 251.

129. H. M. Rosenstock, in "Advances in Mass Spectrometry," Vol. 4, E. Kendrlck, Ed., (The Institute of Petroleum, London, 1968), p. 523. 
130. A. L. Wahrhaftig, in "MTP International Review of Sclence, Volume 5, Mass Spectrometry," A. Maccoll, Ed., (University Park Press, Baltimore, 1972), Chapter 1.

131. M. L. Vestal, in "Fundamental Processes in Radiation Chemistry," P. Ausloos, Ed., (Intersclence, New York, 1968), Chapter 2.

132. S. Glasstone, K. J. Laidler and H. Eyring, "Theory of Rate Processes" (McGraw-H1ll, New York, 1941).

133. R. A. Marcus and O. K. Rice, J. Phys. Collold Chem. 55, 894 (1951).

134. R. A. Marcus, J. Chem. Phys. 20, 359 (I952).

135. F. H. Mies and M. Krauss, J. Chem. Phys. 45, $4455(1966)$.

136. R. D. Levine, J. Chem. Phys. 44, 2046 (1966).

137. J. H. Futrell and T. O. Tiernan, in "Fundamental Processes in Radiation Chemistry," P. Ausloos, Ed., (Interscience, New York, 1968), Chapter 4.

138. P. M. Guyon, W. A. Chupka and J. Berkow1tz, J. Chem. Phys. 64, 1419 (1976).

139. W. A. Chupka, in "Chemical Spectroscopy and Photochemistry in the Vacuum Ultraviolet," C. Sandorfy, P. J. Ausloos and M. B. Robin, Eds., (D. Reldel, Dordrecht-Holland/Boston, 1974), D. 433. 
140. W. A. Chupka and J. Berkowitz, J. Chem. Phys. 47, 2921 ( 1967 ).

141. E. G. Jones, J. H. Beynon and R. G. Cooks, J. Chem. Phys. 57, 3207 (1972).

142. C. E. Klots, J. Chem. Phys. 58, 5364 (1973).

143. S. M. Gordon and N. W. Reid, Int. J. Mass Spect. and Ion Phys. 18, 379 (1975).

144. B. Steiner, C. F. Glese and M. G. Inghram, J. Chem. Phys. 34, 189 (1961).

145. L. Dunoyer, Le Rad1um 8, 142 (1911).

146. I. Estermann, Ed., "Recent Research in Molecular Beams" (Academic Press, New York, 1959).

147. H. Pauly, Fortschr. Physik 9, 613 (1961).

148. N. F. Ramsey, "Molecular Beams" (Oxford Un1versity Press, Oxford, 1956).

149. K. F. Smith, "Molecular Beams" (Methuen and Co., Itd., London, 1955).

150. I. Estermann, Rev. Mod. Phys. I8, 300 (1946).

151. R. G. J. Fraser, "Molecular Beams" (Methuen \& Co., Ltd. London, 1937).

152. R. G. J. Fraser, "Molecular Rays" (Cambridge Univ. Press, London, 1931).

153. J. B. Anderson, R. P. Andres and J. B. Fenn, in "Advances in Atomic and Molecular Physics," Vol. I, D. R. Bates and I. Estermann, Eds., (Academic Press, New York, 1965), D. 345. 
154. T. H. Johnson, Nature 119, 745 (1927).

155. T. H. Johnson, Phys. Rev. 31, 103 (1928).

156. W. H. Rodebush, Rev. Mod. Phys. 3, 392 (1931).

157. A. Kantrowitz and J. Grey, Rev. Sc1. Instr. 22, 328 ( 1951 ).

158. G. B. Kistlakowsky and W. P. Slichter, Rev. Sci. Instr. 22, 333 (1951).

159. E. W. Becker and K. Bier, Z. Naturforsch. 9A, 975 (1954).

160. E. W. Becker and W. Henkes, Z. Phys. 146, 320 (1956).

161. O. Hagena and W. Henkes, Z. Naturforsch. 15A, 851 (1960).

162. J. A. Phipps, D. G. Griffith and J. E. Scott, Jr., Bull. Am. Phys. Soc. $\underline{8}, 550$ (1963).

163. J. B. Anderson and J. B. Fenn, Phys. Fluids $\underline{8}$, 780 (1965).

164. E. L. Knuth, App1. Mech. Rev. 17, 751 (1964).

165. V. B. Leonas, Sov. Phys. - Usp. (Eng. Translation) I, 121 (1964).

166. J. B. Anderson, R. P. Andres and J. B. Fenn, in "Advances in Chemical Physics," Volume X, J. Ross, Ed., (Intersclence, New York, 1966), p. 275.

167. J. B. Anderson, in "Gasdynamics: Molecular Beams. and Low Density Gasdynamics," Vol. 4, P. P. Wegener, Ed., (Marcel Dekker, New York, 1974), p. I. 
168. H. M. Parker, A. R. Kuhl thau, R. Zapata and J. E. Scott, Jr., in "Proc. Ist Intern. Symp. Rarefied Gas Dynamics, Nice, 1958," F. M. Devienne, Ed., (Pergamon, New York, 1960), p. 69.

169. U. Bossel, Ph.D. Thesis, The University of California, Berkeley, 1968.

170. J. B. Fenn and J. B. Anderson, in "Proc. 4th Intern. Symp. Rarefied Gas Dynamics, Toronto, 1964," J. H. de Leeuw, Ed., (Academic Press, New York, 1966), p. 311 .

171. R. Campargue, Entropie 30, 15 (1969).

172. T. R. Govers, R. L. LeRoy and J. M. Deckers, in "Proc. 6th Intern. Symp. Rarefled Gas Dynamics, Cambridge, 1968," L. Triliing and H. Y. Wachman, Eds. (Academic Press, New York, 1969), p. 985.

173. G. A. Bird, Phys. Fluids 19, 1486 (1976).

174. K. Bier and O. F. Hagena, in "Proc. 4th Intern. Symp. Rarefied Gas Dynamics, Toronto, 1964," J. H. de Leeuw, Ed. (Academic Press, New York, 1966), p. 260 .

175. U. Bossel, F. C. Hurlbut and F. S. Sherman, in "Proc. 6th Intern. Symp. Rarefied Gas Dynamics, Cambridge, 1968," L. Trilling and H. Y. Wachman, Eds. (Academic Press, New York, 1969), p. 945. 176. U. Bossel, Entropie 42, 12 (1971). 
177. W. R. Gentry and C. F. Glese, Rev. Sc1. Instrum. 46, 104 (1975).

178. R. Campargue, J. Chem. Phys. 52, 1795 (1970).

179. P. A. Tahourdin, "Final Report on the Jet Separation Method," Oxford Rept. $36 \mathrm{Br} .694$, Clarendon Laboratory, Oxford, England, 1946. 180. V. H. Rels and J. B. Fenn, J. Chem. Phys. 39, 3240 (1963).

181. F. Zigan, Z. Naturforsch. I7A, 772 (1962).

182. H. Mikami and Y. Takashima, Bull. Tokyo Inst. Tech. $\underline{61}, 67$ (1964).

183. F. S. Sherman, Phys. Flusds 8,773 (1965).

184. H. Mikam1 and Y. Takash1ma, Int. J. Heat Mass Transfer 11, 1597 (1968).

185. D. I. Sebacher, AIAA J. $\underline{6}, 51$ (1968).

186. R. Campargue, Entropie 18, 55 (1967).

187. E. P. Muntz, B. B. Hamel and P. B. Scott, Entrople 42, 28 (1971).

188. R. Campargue, J. B. Anderson, J. B. Fenn, B. B. HameI, E. P. Muntz and J. R. Wh1te, Entropie 1.2, (67), 11 (1976).

189. R. Campargue and A. Lebehot, in "Proc. 9 th Intern. . Symp. Rarefied Gas Dynamics, Gottingen, 1974," M. Becker and M. Fleb1g, Eds. (DFVLR-Press, PorzWahn, 1974), C. 11. 
190. O. F. Hagena, in "Gasdynam1cs: Molecular Beams and Low Density Gasdynamies," Vol. 4, P. P. Wegener, Ed. (Marcel Dekker, New York, 1974), p. 93.

191. O. F. Hagena, Phys. Fluids 17, 894 (1974).

192. O. F. Hagena and W. Obert, J. Chem. Phys. 56, 1793 (1972).

193. 0. F. Hagena, Entropie 42, 42 (1971).

194. A. B. Balley, M. R. Busby and R. Dawbarn, "Cluster Formation in Free-Jet Expansions." AEDC-TR-72-32 (AD-740898), Apri1 1972.

195. D. Golomb, R. E. Good, A. B. Balley, M. R. Busby and R. Dawbarn, J. Chem. Phys. 57, 3844 (1972).

196. D. Golomb, R. E. Good and R. F. Brown, J. Chem. Phys. 52, 1545 (1970).

197. T. A. Milne, A. E. Vandegrift and F. T. Greene, J. Chem. Phys. 52, 1552 (1970).

198. T. A. Milne, J. E. Beachey and F. T. Greene, J. Chem. Phys. 57, 2221 (1972).

199. A. B. Balley, M. R. Busby and R. Dawbarn, "Effest of Skimmer Interaction on the Properties of Partially Condensed Molecular Beams." AEDC-TR-72-100 (AD-746292), August 1972 .

200. B. L. Blaney and G. E. EwIng, Ann. Rev. Phys. Chem. 27, 553 (1976). 
201. D. J. Royer, "Bonding Theory" (McGraw-H1ll, New York, 1968), Chapter 8.

202. D. L. KIng, D. A. Dixon and D. R. Herschbach,

J. Am. Chem. Soc. 96, 3328 (1974).

203. D. A. Dixon and D. R. Herschbach, J. Am. Chem. Soc. 97, 6268 (1975).

204. A. G. Ureña, R. B. Bernstein and G. R. Phillips, J. Chem. Phys. 62, 1818 (1975).

205. R. Behrens, Jr., A. Freedman, R. R. Herm and T. P. Parr, J. Chem. Phys. 63, 4622 (1975).

206. G. E. Ewing, Acc. Chem. Res. 8, 185 (1975).

207. W. Klemperer, Ber. Bunsenges. 78,128 (1974).

208. G. E. Ewing, Angew. Chem. Internat. Edit. (English) 11, 486 (1972).

209. James A. R. Samson, "Techniques of Vacuum Ultraviolet Spectroscopy" (W1ley, New York, 1967).

210. R. E. Huffman, J. C. Larrabee and Y. Tanaka, Appl. Opt1cs 4, 1581 (1965).

21I. R. P. Madden, D. L. Ederer and K. Codling, AppI. Optics $\underline{6}, 31$ (1967).

212. J. W. Taylor, in "Chemlcal Spectroscopy and Photochemistry in the Vacuum-Ultraviolet," C. Sandorfy, P. J. Ausloos and M. B. Robin, Eds. (D. Reldel, Dordrecht-Holland/Boston, 1974), p. 543. 
213. R. E. Watson and M. I. Perlman, Sclence 199, 1295 (1978).

214. M. Seya, Sc1. Light (Tokyo) 2, 8 (1952).

215. T. Namioka, Sc1. Light (Tokyo) 3 , 15 (1954).

216. T. Namioka, J. Opt. Soc. Am. 49, 951 (1959).

217. Reference 209, pp. 62-64.

218. K. L. Bath and B. Brehm, Z. Angew. Phys. 19, 39 (1965).

219. W. C. Walker, N. Wainfan and G. L. Weissler, J. Appl. Phys. 26, 1366 (1955).

220. R. W. Kiser, "Introduction to Mass Spectrometry and its Applications" (Prentice-Hall, Englewood Cl1ffs, N. J., 1965).

221. C. A. McDowell, Ed., "Mass Spectrometry" (McGrawH1II, New York, 1963).

222. W. Paul and M. Raether, Z. Phys. 140, 262 (1955).

223. P. H. Dawson and N. R. Whetten, Advanc. In Electronics and Electron Phys. 27, 59 (1069).

224. W. M. Brubaker, in "Proc. 5th Intern. Instruments and Measurements Conf., Stockholm, 1960,"

H. Von Koch and G. Ljungberg, Eds. (Academic, New York, 1961), p. 305.

225. E. Schơnheit, Z. Physik 149, 153 (1957).

226. E. Schönhe1t, Z. Angew. Phys. 2, 171 (1957). 
227. C. F. Glese, Rev. Sc1. Instrum. 30, 260 (1959).

228. C.-S, Lu and H. E. Carr, Rev. Sc1. Instrum. 33, 823 (1962).

229. N. R. Daly, Rev. Sc1. Instrum. 31, 264 (1960).

230. M. W. P. Cann, App. Opt1cs 8 , 1645 (1969).

231. J. A. R. Samson, J. Opt. Soc. Am. 54, 6 (1964).

232. RCA Tube Handbook HB-3, Photosensitive Device Section, 6199 .

233. S. Dushman, "Scientific Foundations of Vacuum Techntque" (Wiley, New York, 1949).

234. A. B. Balley, "Cluster Intensity and Velocity

Measurements in Condensed Flows." AEDC-TR-74-38 (AD-785348), September 1974.

235. J. A. Beran and I. Kevan, J. Phys. Chem. 73, 3866 (1969).

236. J. A. Beran, A. K. Dhingra, W. -S. Liu and D. F. Mullica, Can. J. Chem. 53, 1616 (1975).

237. V. K. Potapov, A. D. Filyugina, D. N. Shigorin and G. A. Ozerova, DokI. Akad. Nauk SSSR I80, 398 (1968).

238. G. Herzberg and L. L. Howe, Can. J. Phys. 37 , 636 (1959).

239. G. Mouvier and R. Hernandez, Org. Mass Spectrometry 10, 958 (1975). 
240. R. A. W. Johnstone, F. A. Melion and S. D. Ward, Int. J. Mass Spect. Ion Phys. 5, 241 (1970).

241. M. A. Haney and J. I. Franklin, Trans. Faraday Soc. 65, 1794 (1969).

242. K. Hirota and M. Hatada, Kinetika 1 Kataliz $\underline{8}$, 748 (1967).

243. V. K. Potapov and D. N. Shlgorin, Zh. Flz. Khim. 40, 200 (I966).

244. D. N. Sh1gorin, A. D. Filyugina and V. K. Potapov, Teor. I Eksperim. Khim. 2, 554 (1966).

245. F. H. Dorman, J. Chem. Phys. 42, 65 (1965).

246. A. Foffant, S. Pignataro, B. Cantone and F. Grasso, Z. Physik Chem. (Frankfurt) 42, 221 (1964).

247. A. Foffan1, S. PIgnataro, B. Cantone and F. Grasso, Nuovo Cimento 29, 918 (1963).

248. I. Kanomata, Bull. Chem. Soc. Japan 34, 1854 (1961).

249. J. R. Majer, C. R. Patrick and J. C. Robb, Trans. Faraday Soc. 57, 14 (1961).

250. A. B. King and F. A. Long, J. Chem. Phys. 29, 374 (1958).

251. R. I. Reed and J. C. D. Brand, Trans. Faraday Soc. 54, 478 (I958).

252. K. Higas1, I. Omura, and H. Baba, Nature I78, $652(1956)$. 
253. I. Omura, K. H1gasl and H. Baba, Bull. Chem. Soc. Japan 29, 504 (1956).

254. J. D. Morrison and A. J. C. Nicholson, J. Chem. Phys. 20, 1021 (1952).

255. E. Murad and M. G. Inghram, J. Chem. Phys. 40, 3263 (1964).

256. E. Murad and M. G. Inghram, J. Chem. Phys. 4I, 404 (1964).

257. F. I. Vilesov and A. N. Terenin, DokI. Akad. Nauk SSSR II5, 744 (1957).

258. R. Hernandez, P. Masclet and G. Mouvier, J. Electron Spect. Related Phenom. 10, 333 (1977).

259. V. K. Potapov. G. V. Karachevtsev and M. M. I1pe1, Kh1m. Vys. Energ. 1I, 107 (1977).

260. V. Y. Young and K. L. Cheng, J. Chem. Phys. 65, 3187 (1976).

26I. K. Kimura, S. Katsumata, T. Yamazaki and $\mathrm{H}$. Wakabayash1, J. Electron Spect. Related Phenom. 白, 41 (1975).

262. G. Hentrich, E. Gunkel and M. Klessinger, J. Mol. Structure 21, 231 (1974).

263. J. Kelder, H. Cerfontaln, B. R. Higginson and D. R. Lloyd, Tetrahedron Letters, 739 ( 1974 ).

264. H. Ogata, J. Kitayama, M. Koto, S. KoJ1ma, Y. Nihe1 and H. Kamada, EuII. Chem. Soc. Japan 4 T, 958 (1974). 
265. W.-C. Tam, D. Yee and C. E. Brion, J. Electron Spec. Related Phenom. 4, 77 (1974).

266. C. R. Brundle, M. B. Rob In, N. A. Kuebler and H. Basch, J. Amer. Chem. Soc. 94, 1451 (1972).

267. G. V. Karachevtsev, V. K. Potapov and V. V. SorokIn, DokI. Akad. Nauk SSSR 206, 144 (1972).

268. A. W. Potts, T. A. W1lliams and W. C. Price, Faraday Discussions Chem. Soc. 1972, No. 54, pp. 104-15.

269. B. J. Cocksey, J. H. D. Eland and C. J. Danby, J. Chem. Soc. (B), 790 (1971).

270. M. J. S. Dewar and S. D. Worley, J. Chem. Phys. 50, 654 (1969).

271. M. I. Al-Joboury and D. W. Turner, J. Chem. Soc., 4434 (1964).

272. R. Stockbauer, Int. J. Mass Spect. Ion Physics $\underline{25}$, 89 (1977).

273. C. S. T. Cant, C. J. Danby and J. H. D. Eland, J. Chem. Soc. Faraday Trans. 2 71, 1015 (1975).

274. D. S. C. Yee and C. E. Brion, J. Electron Sp-at. Related Phenom. 8, 377 (1976).

275. V. Cermak, Collection Czech. Chem. Commun. 33, 2739 (1968).

276. M. A. Slifkin and A. C. Allison, Nature 215, $949(1967)$. 
277. R. H. Huebner, R. J. Celotta, S. R. Mlelczarek and C. E. Kuyatt, J. Chem. Phys. 59, 5434 (1973). 278. J. H. Beynon, A. E. Fontaine and B. E. Job, Z. Naturforschg. 2la, 776 (1966).

279. Z. Luczynski and H. Wincel, Int. J. Mass Spec. Ion Phys. 23, 37 (19/77).

280. A. S. Blair and A. G. Harrison, Can. J. Chem. 51, 703 (1973).

281. L. W. Sleck and P. Ausloos, Radiat. Res. 52, 47 (1972).

282. K. A. G. MacNell and J. H. Futrell, J. Phys. Chem. 76, 409 (1972).

283. J. O. Terry and T. O. Tiernan, Proc. 16th Ann. Conference on Mass Spectrometry and Allied Toplcs, ASTM Committee EI4, P1ttsburgh, Pa., 1968, D. 33. 284. V. K. Potapov, DokI. Akad. Nauk SSSR 183, 386 (1968). 285. M. S. B. Munson, J. Amer. Chem. Soc. 87, 5313 (1965). 286. R. S. Mulliken, J. Chem. Phys. 3, 564 (1935). 287. J. Chao and B. J. Zwolinski, J. Phys. Chem. Ref. Data 5, 319 (1976).

288. G. Dellepiane and J. Overend, Spectrochim. Acta 22, $593(1966)$.

289. R. S. Mulliken, J. Chem. Phys. 52, 5170 (1970). 290. C. Braun and H. Leidecker, J. Chem. Phys. 6I, $3104(1974)$. 
291. J. O. Hirschfelder, C. F. Curtiss and R. B. Bird, "Molecular Theory of Gases and Liquids" (W1ley, New York, 1954).

292. R. F. HajJar, W. B. Kay and G. F. Leverett, J. Chem. Eng. Data 14, 377 (1969).

293. J. H. Dymond and E. B. Smith, "The Virial Coefficients of Gases" (Clarendon, Oxford, 1969), D. 76.

294. J. D. Lambert, G. A. H. Roberts, J. S. Rowlinson and V. J. Wilkinson, Proc. Roy. Soc. A196, 112 (1949).

295. J. S. Rowlinson, Trans. Faraday Scc. 45, 974 (1949).

296. I. N. Anderson, A. P. Kudchadker and P. T. Eubank, J. Chem. Eng. Data 13, 321 (1968).

297. E. J. Robbins, R. E. Leckenby and P. Willis, Adv. Phys. 16, 739 (1967).

298. A. Herrmann, E. Schumacher and L. Wöste, J. Chem. Phys. 68, 2327 (1978).

299. P. J. Harbour, J. Phys. B 4, 528 (1971). 300. W. T. Simpson, "Theories of Electrons in Molecules" (Prentice-Hall, Englewood Cliffs, N. J., 1962), Chapter 4.

301. W. E. Splcer, in "Optical Properties of Solids:

New Developments," B. O. Seraph1n, Ed. (Amerlcan Elsevier, New York, 1976), pp. 667-671.

302. K. Y. Yu, J. C. McMenamin and W. E. Splcer, Surface Science 50, 149 (1975). 
303. W. C. Price and D. M. Simpson, Proc: Roy. Soc. (London) Al65, 272 (1938).

304. Y. Tanaka, A. S. Jursa and F. J. LeBlanc, J. Chem. Phys. 32, 1205 (1960).

305. J. Momigny and J. Delwiche, J. Chim. Phys. Physiochim. B101. 65, 1213 (1968).

306. A. W. Potts and T. A. Williams, J. Electron Spect. Related Phenom. 3, 3 (1974).

307. T. A. Carlson and G. E. McGuire, J. Electron Spect. Filated Phenom. I, 209 (1973).

308. C. R. Brundle and D. W. Turner, Int. J. Mass Spect. Ion Phys. 2, 195 (1969).

309. J. E. Collin and P. Natalis, Int. J. Mass Spect. Ion Phys. I, 121 (1968).

310. M. I. AI-Joboury, D. P. May and D. W. Turner, J. Chem. Soc.,6350 (1965).

311. Reference 93, Table 64.

312. M. Meot-Ner and F. H. Field, J. Chem. Phys. 66, 4527 (1977).

313. K. C. Janda, J. C. Hemminger, J. S. Winn, S. E. Novick, S. J. Harris and W. Klemperer, J. Chem. Phys. 63, 1419 (1975). 
314. K. Bowen, R. Cavanagh, R. Altman, K. Chance, T. Dixon, S. Holmgren, C. Joyner, K. Leopold, J. Lisy, J. Steed, J. Winn and W. Klemperer, "Polarity of Substituted Benzene Molecules," 33rd Symposium on Molecular Spectroscopy, Oh10 State UnIversity, June 12-16, 1978.

315. M. Meot-Ner and F. H. Fleld, J. Am. Chem. Soc. 99, 998 (1977).

316. F. C. Fehsenfeld, C. J. Howard, W. J. Harrop and E. F. Ferguson, J. Geophys. Res. 80, 2229 (1975).

317. G. C. Reid, in "Advances in Atomic and Molecular Physles," Vol. I2, D. R. Bates and B. Bederson, Ed. (Academ1c Press, New York, 1976), p. 375. 


\section{ACKNOWLEDGMENTS}

I am especially grateful for the guidance and support of my thesis advisor, Edward A. Walters. Special thanks extend to my site advisor at the Los Alamos Scientific Laboratory, Normand C. Blais. In addition, I wish to acknowledge much helpful advice from John Sullivan, Gerald Streit, Jon Cross, and other members of Group CNC-2 at Los Alamos Scientific Laboratory.

I would lire to thank Richard Keller for suggesting the independent systems model for the acetone cluster ion system and James Stine for his instructive calculations on the same. I also wish to express my appreciation to Berlyn Brixner of Group M-I at Los Alamos Scientific Laboratory for the design and fabrication of the grazing incidence alignment mirror. Several very helpful telephone conversations Nith James A. R. Samson and Nililam Chupka are aprreciated $a \approx$ weIl. Thanks also extend to P. W. Tiedemann and S. T. Deyer :or some profitable conversations at Berkeley in August 1977. Funding from the Associated Western Universities, Inc. is gratefully acknowledged. This research was done with support from the US Derartmen: o: Energy. I am deeply indebted to Dolores Blumenthal colby for her tyoing of this manuscrict. 